\title{
Processing Quantum Signals Carried by Electrical Currents
}

\author{
B. Rousselø, ${ }^{1,2}$ C. Cabart, ${ }^{1}$ G. Fève $\odot,{ }^{3}$ and P. Degiovanni® ${ }^{1, *}$ \\ ${ }^{1}$ Université Lyon, ENS de Lyon, Université Claude Bernard Lyon 1, CNRS, Laboratoire de Physique, Lyon \\ F-69342, France \\ ${ }^{2}$ European Space Agency - Advanced Concepts Team, ESTEC, Keplerlaan 1 Noordwijk 2201 AZ, Netherlands \\ ${ }^{3}$ Laboratoire de Physique de l'Ecole Normale Supérieure, ENS, Université PSL, CNRS, Sorbonne Université, \\ Université de Paris, F-75005 Paris, France
}

(Received 4 August 2020; accepted 26 January 2021; published 4 May 2021)

\begin{abstract}
Recent developments in the coherent manipulation of electrons in ballistic conductors include the generation of time-periodic electrical currents involving one to few electronic excitations per period. However, using individual electrons as carriers of quantum information for flying qubit computation or quantum metrology applications calls for a general method to unravel the single-particle excitations embedded in a quantum electrical current and how quantum information is encoded within it. Here, we propose a general signal-processing algorithm to extract the elementary single-particle states, called electronic atoms of signal, present in any periodic quantum electrical current. These excitations and their mutual quantum coherence describe the excess single-electron coherence in the same way musical notes and score describe a sound signal emitted by a music instrument. This method, which is the first step towards the development of signal processing of quantum electrical currents is illustrated by assessing the quality of experimentally relevant single electron sources. The example of randomized quantum electrical currents obtained by regularly clocked but randomly injected unit-charge Lorentzian voltage pulses enables us to discuss how interplay of the coherence of the applied voltage and the Pauli principle alter the quantum coherence between the emitted single-particle excitations.
\end{abstract}

DOI: 10.1103/PRXQuantum.2.020314

\section{INTRODUCTION}

These recent years have seen a spectacular breakthrough in the manipulation of quantum electric circuits. Ondemand single-electron sources (SESs) in quantum Hall edge channels [1-4], two-dimensional (2D) electron gases using electron pumps [5] or surface acoustic waves [6] and in tunnel junctions [7] enable us to engineer timedependent quantum electrical currents involving one to a few elementary excitations per period. This emerging field, called electron quantum optics, precisely aims at generating, manipulating, and characterizing such "quantum beams of electricity" in metallic quantum conductors [8]. The latest advances have given access to the single-particle wave functions carried by such quantum electrical currents together with their emission probabilities and coherences [9] thereby demonstrating our ability to access electronic

*pascal.degiovanni@ens-lyon.fr

Published by the American Physical Society under the terms of the Creative Commons Attribution 4.0 International license. Further distribution of this work must maintain attribution to the author(s) and the published article's title, journal citation, and DOI. quantum states at an unprecedented level. These achievements strongly suggest that this field is now sufficiently mature for exploring its applications.

From a quantum technology point of view, quantum electrical currents carry quantum information through their single-, two-, and ultimately, many-particle content. For example, single electrons delocalized on two onedimensional channels have been proposed as "railroad flying qubit" [10-14] in which a qubit state is encoded in the quantum delocalization of an electron on two copropagating one-dimensional (1D) channels [15]. This is a very promising line of research towards the development of quantum spintronics $[16,17]$ and, in the longer term, of free-electron quantum computation [18-20].

This information can be accessed through a hierarchy of electronic coherences similar to the ones introduced by Glauber [21] for photons. These coherences are the "quantum signals" carried by the quantum electrical current in a metallic conductor. Because of the parity superselection rule [22-24], the first nonzero electronic quantum signal is the single-electron coherence $[25,26]$ containing all information on single-particle excitations within the system. The next one is the second-order electronic coherence $[27,28]$ that describes two-particle excitations within the beam. 
Measuring these quantum signals requires quantum tomography protocols for $n$-electron coherence. Such tomography protocols are all based on the transformation of quantum signals into measurable quantities. For example, Mach-Zehnder, Hong-Ou-Mandel (HOM) and Franson electron interferometry experiments realize "filtering" or "overlaps" on electronic coherences [29], thereby encoding the results of these operations into experimentally accessible quantities such as average current [30] and current correlations [31,32]. Electronic HOM interferometry [33] is at the core of the recently demonstrated HOM single-electron tomography [9,34], whereas, for higherenergy (meV) electrons, a time-dependent quantum point contact (QPC) is used as a time-dependent energy filter for reconstructing single-electron coherence [35].

This, however, leaves open the question of decoding classical or quantum information encoded within quantum electrical currents. This requires finding appropriate representations of electronic coherences. In the present context, "appropriate" means simple with respect to the reference state, which is a Fermi sea at a given chemical potential. We, therefore, consider the excess single-particle coherence describing the single-particle content in terms of electron and hole excitations with respect to the reference Fermi sea. Ideally, we are looking for the simplest possible description, requiring minimal data to encode this description of the single-particle content.

In this paper, we show in full generality that such a description exists: any excess time-periodic single-electron coherence admits a minimal description in terms of quasiperiodic single-electron and single-hole excitations, which are the time-domain counterparts of Bloch waves in solid-state physics [36]. This implies that only electron and hole Bloch-wave emission probabilities as well as electron-hole coherences between two different Bloch waves are required to know the single-electron coherence.
Considering the counterpart of Wannier functions [37], which are localized wave functions contrary to Bloch waves, the excess single-electron coherence can then be expressed in terms of a set of mutually orthogonal singleparticle states called electronic atoms of signal [29] thereby providing us with a discrete description of the electronic coherence. We see that electronic atoms of signals and the discrete representation of single-electron coherence can be viewed as the counterpart of music notes and of a musical score as pictured on Fig. 1. Therefore, the extraction of such a simple form of single-electron coherence provides us with the appropriate toolbox to develop a full wavepacket-based approach on quantum transport envisioned in pioneering works [41,42]. In a broader perspective, it is a crucial step in the development of "quantum signal processing" for quantum electrical currents that extends the general paradigm of signal processing [43] to the quantum realm. It would entitle us with an enabling set of technologies and methods aiming at encoding, transferring, and retrieving quantum information carried by these "quantum signals," a crucial step for the applications of electronbased quantum technologies.

Whenever interactions can be neglected, this description can be used to describe the full many-body state of the electron fluid and, therefore, to access many-particle quantities such as the electron-hole entanglement entropies. This connection can be made explicit using time-periodic single-particle scattering theory and has been used to obtain the full counting statistics of single-particle excitations [44].

The entanglement entropy inferred from this representation of single-electron coherence can then be used to assess the quality of experimentally relevant singleelectron sources such as the mesoscopic capacitor (MC). We are also able to obtain an explicit description of the single-electron excitations emitted. Finally, in order

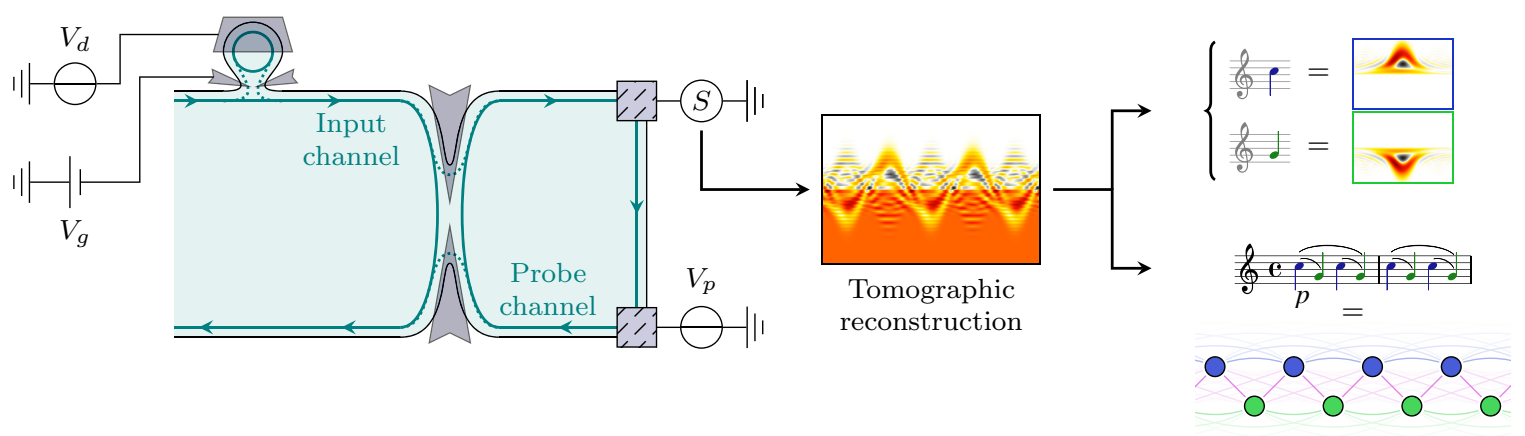

FIG. 1. Schematic of the process for extracting the single-particle content from a quantum electrical current. Left: the Hong-OuMandel interferometer uses two-particle interferences to encode the overlap between the injected single-electron coherences into the outgoing current noise [29]. Middle: the single-electron coherence is reconstructed from current noise measurements [34,38,39]. Right: the result of the tomographic reconstruction, depicted here as the electronic Wigner distribution function [40], is processed by the algorithm described in the present paper to obtain a description of single-electron coherence in terms of electronic atoms of signal (counterparts of musical notes) arranged according to a "quantum coherence score" (counterpart of the music score). 
to illustrate the possibility for modulating emission probabilities and coherences, we apply our algorithm to the recently introduced randomized trains of Lorentzian pulses [45], an interesting example that enlightens the role of the Pauli exclusion principle in electronic quantum signals.

This paper is structured as follows: in Sec. II, we introduce the problem of finding a simple representation of single-electron coherence. Then, in Sec. III, we present our algorithm for finding such a representation for any time-periodic excess single-electron coherence. The relation of this representation to electron-hole entanglement is discussed in Sec. IV. Finally, we apply our method to the study of the mesoscopic capacitor to assess its quality as a single-electron source, and to periodic and randomized Leviton trains in Sec. V.

\section{STATEMENT OF THE PROBLEM}

Let us now introduce and motivate the problem considered here by considering simple trains of excitations used to model the emission by experimentally demonstrated single to few electron sources. These simple examples enable us to write down a simple representation of the excess single-electron coherence, a generalization of which is shown to exist in Sec. III.

\section{A. Electronic coherence}

The central concept of electron quantum optics are the electronic coherences defined by analogy with Glauber's coherences of photon quantum optics [21]. They carry all the information on the fermionic $n$-particle states propagating within the conductor. Here we focus on singleelectron coherence, which, at position $x$ along a single chiral electronic channel, is defined as $[25,38]$

$$
\mathcal{G}_{\rho, x}^{(e)}\left(t \mid t^{\prime}\right)=\operatorname{tr}\left[\psi(x, t) \rho \psi^{\dagger}\left(x, t^{\prime}\right)\right]
$$

where $\rho$ denotes the many-body reduced density operator for the electron fluid and $\psi$ the fermionic field operator describing the electrons. When all the electronic sources are switched off, $\mathcal{G}_{x, \text { off }}^{(e)}$ coincides with the equilibrium single-electron coherence characterized by a chemical potential $\mu$ and an electronic temperature $T_{\mathrm{el}}$. When sources are switched on, the excess single-electron coherence defined by $\mathcal{G}_{x, \text { on }}^{(e)}=\mathcal{G}_{x, \text { off }}^{(e)}+\Delta \mathcal{G}_{x}^{(e)}$ contains all the information on the single-particle excitations generated by the sources and drives that are switched on.

The single-electron coherence can be studied in the time domain as well as in the frequency domain but is most conveniently visualized using a real-valued time-frequency representation called the Wigner distribution function [40]

$$
W_{\rho, x}^{(e)}(t, \omega)=\int_{\mathbb{R}} v_{F} \mathcal{G}_{\rho, x}^{(e)}\left(t+\frac{\tau}{2} \mid t-\frac{\tau}{2}\right) e^{i \omega \tau} d \tau .
$$

\section{B. Electron and hole trains}

An ideal periodic single-electron source is a periodically operated device that emits exactly one single-electron excitation on top of the Fermi sea $\left|F_{\mu=0}\right\rangle$ during each period. The corresponding many-body state is an electron train of the form

$$
\left|\Psi_{\mathrm{SES}}\right\rangle=\prod_{l \in \mathbb{Z}} \psi^{\dagger}\left[\varphi_{e, l}\right]\left|F_{\mu=0}\right\rangle,
$$

where $\psi^{\dagger}\left[\varphi_{e, l}\right]$ creates a single-particle excitation in the electronic wave function $\varphi_{e, l}$. It differs from $\varphi_{e, l=0}$ by translation by $l T$ in the time domain. Ideally, one would like each of these electronic excitations to be perfectly distinguishable from each other, which means that $\varphi_{e, l}$ and $\varphi_{e, l^{\prime}}$ are orthogonal when $l \neq l^{\prime}$. In this case, the excess single-electron coherence is

$$
\Delta \mathcal{G}^{(e)}\left(t \mid t^{\prime}\right)=\sum_{l=-\infty}^{+\infty} \varphi_{e, l}(t) \varphi_{e, l}\left(t^{\prime}\right)^{*}
$$

For example, when driven by a square gate voltage $V_{g}(t)$ and for a suitable value of the dot transparency $D=D_{\text {opt }}$ the mesoscopic capacitor depicted on Fig. 2 ideally generates one electron excitation and one hole excitation per period $[1,46]$ :

$$
\left|\Psi_{\mathrm{MC}-\mathrm{SES}}\right\rangle=\prod_{l=-\infty}^{+\infty} \psi^{\dagger}\left[\varphi_{e, l}\right] \psi\left[\varphi_{h, l}\right]\left|F_{\mu=0}\right\rangle
$$

Here $\varphi_{e, l}$ and $\varphi_{h, l}$ are time translated by $l T$ from the emitted electron $\varphi_{e, 0}$ and hole wave functions $\varphi_{h, 0}$ and are mutually orthogonal and normalized. The excess single-electron coherence is then given by

$$
\Delta \mathcal{G}^{(e)}=\sum_{l=-\infty}^{+\infty} \varphi_{e, l}(t) \varphi_{e, l}\left(t^{\prime}\right)^{*}-\sum_{l=-\infty}^{+\infty} \varphi_{h, l}(t) \varphi_{h, l}\left(t^{\prime}\right)^{*}
$$

where the hole contribution naturally comes with a minus sign.

When closing the dot, the time needed to emit an electronic (or hole) excitation becomes larger than $T / 2$. It was argued [38] that the mesoscopic capacitor emits a quantum superposition of no excitation and an elementary electron and hole pair, thereby leading to electron-hole coherences visible on Fig. 5 of Ref. [38]. Assuming that, for each period, the corresponding electron-hole pair involves only electronic atoms of signals associated with this specific 


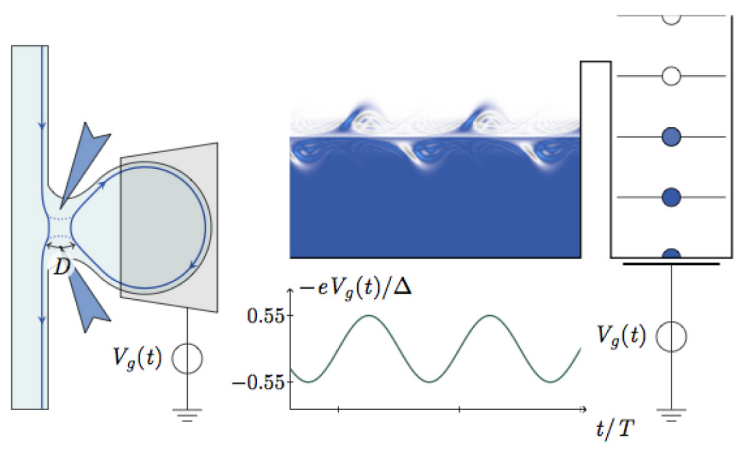

FIG. 2. Left panel: The mesoscopic capacitor is a ballistic quantum conductor formed by connecting a quantum dot to a chiral edge channel via a quantum point contact of transparency $D$. Right panel: Modelization as a driven quantum dot with level spacing $\Delta$ connected to an electronic reservoir. The mesoscopic capacitor is driven by an ac voltage drive $V_{g}(t)$ applied to the top gate. Applying a dc voltage bias to the top gate shifts the energy levels of the dot. The mesoscopic capacitor emits a stream of electron and hole excitations whose Wigner distribution function $W_{S}^{(e)}(t, \omega)$ is depicted as a density plot on the right panel.

period, such a state would be parametrized by

$$
\left|\Psi_{e / h}(u, v)\right\rangle=\prod_{l=-\infty}^{+\infty}\left[u+v \psi^{\dagger}\left(\varphi_{e, l}\right) \psi\left(\varphi_{h, l}\right)\right]\left|F_{\mu=0}\right\rangle,
$$

where $|u|^{2}+|v|^{2}=1$. The resulting single-electron coherence is then

$$
\Delta \mathcal{G}^{(e)}\left(t \mid t^{\prime}\right)=\sum_{l \in \mathbb{Z}}\left[|v|^{2} \varphi_{e, l}(t) \varphi_{e, l}\left(t^{\prime}\right)^{*}-|v|^{2} \varphi_{h, l}(t) \varphi_{h, l}\left(t^{\prime}\right)^{*}\right]
$$

$$
+\sum_{l \in \mathbb{Z}}\left[u v^{*} \varphi_{e, l}(t) \varphi_{h, l}\left(t^{\prime}\right)^{*}+v u^{*} \varphi_{h, l}(t) \varphi_{e, l}^{*}\left(t^{\prime}\right)\right]
$$

in which the rhs of Eq. (8a) lives in the quadrants of electron and hole excitations (see Fig. 3), whereas Eq. (8b) represents the electron-hole pair coherence arising from $\left|\Psi_{e / h}(u, v)\right\rangle$ whenever $u v \neq 0$. Equation (6) is recovered for $(u, v)=(0,1)$, which should, therefore, correspond to $D \simeq D_{\text {opt }}$ whereas for $(u, v)=(1,0)$ one recovers the Fermi sea, the result expected when the dot is totally closed $(D=0)$. The case where $|u|^{2}=|v|^{2} \simeq 1 / 2$ could thus be viewed as the excess electronic coherence from an ideal source emitting a coherent superposition of "nothing" and of a single electron-hole pair per period. It corresponds to maximal electron-hole entanglement [29]. Note, however, that the rhs of Eq. (8) does not involves interperiod coherences (terms with $l \neq l^{\prime}$ ) as a result of the ansatz described by Eq. (7). But, as we see in Sec. V A, this is not the case for the mesoscopic capacitor in the low $D$ regime.

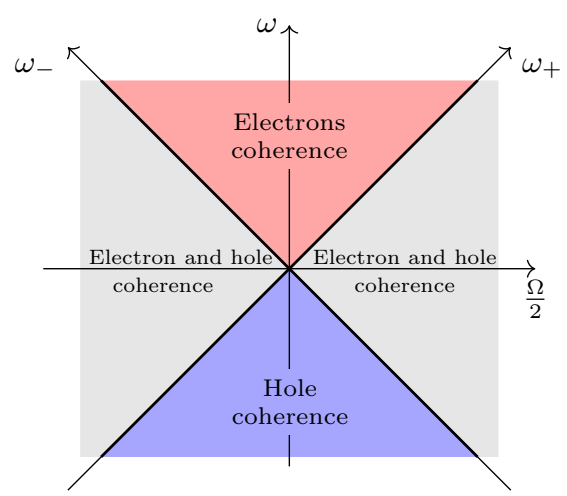

FIG. 3. Frequency-domain quadrants for single-electron coherence: depending on the signs $\left(\varepsilon_{+}, \varepsilon_{-}\right)$of $\left(\omega_{+}, \omega_{-}\right)$, we are considering the matrix elements $\left\langle\omega_{-}\left|\mathbf{G}^{(e)}\right| \omega_{+}\right\rangle$of $\mathbf{G}_{\varepsilon_{+}, \varepsilon_{-}}^{(e)}$ in the $|\omega\rangle$ basis of plane waves (see Appendix A for normalizations). The electronic quadrant (in red) defined by both $\omega_{+}=\omega+\Omega / 2$ and $\omega_{-}=\omega-\Omega / 2$ positive gives information about electronic excitations. The hole quadrant (in blue) defined by both $\omega_{+}$and $\omega_{-}$being negative gives information about hole excitations. The two electron and hole quadrants $\left(\omega_{+} \omega_{-}<0\right.$, light gray) contain information about electron-hole coherences.

The analysis presented in Sec. III indeed enables us to understand quantitatively all the features of single-electron coherence in a simple way.

\section{Electronic atoms of signal}

Equations (4), (6), and (8) correspond to ideal sources and have a simple expression in terms of a family of single-electron wave functions called electronic atoms of signal [29]. Electronic atoms of signal consist of a family of normalized mutually orthogonal single-electron wave functions $\varphi_{a, l}$, which are translated by multiples of $T$ :

$$
\begin{aligned}
& \varphi_{a, l}(t)=\varphi_{a, 0}(t-l T), \\
& \left\langle\varphi_{a, l} \mid \varphi_{a^{\prime}, l^{\prime}}\right\rangle=\delta_{l, l^{\prime}} \delta_{a, a^{\prime}} .
\end{aligned}
$$

Although a decomposition of the form of Eq. (4) is known [47] for a $T$-periodic train of Lorentzian voltage pulses of unit charge at zero temperature, realistic sources are, in general, not ideal. Even the forms given by Eqs. (6) and (8) are too simple to describe the excess single-electron coherence of all experimentally realistic sources. First of all, even at very low temperature, they correspond to ideal operating regimes, which are only asymptotic with respect to the experimental parameters as in the case of the mesoscopic capacitor at $D \simeq D_{\text {opt }}$. Moreover, at nonzero temperature $T_{\mathrm{el}}$, electron-hole pairs are generated from thermal fluctuations and introduce an underlying thermal coherence time $\hbar / k_{B} T_{\text {el }}$ a priori unrelated to the period $T$. It may lead to interperiod coherences not present in Eq. (8). Last but not least, when electronic coherence is measured 
at some distance from such a source, Coulomb interactions alter the electronic coherence in a drastic way [48-50], leading to extra electron-hole pairs [51].

These remarks raise the question of finding a way to express an arbitrary periodic single-electron coherence in terms of suitable electronic atoms of signals. We now present a systematic procedure for obtaining such an expression together with the appropriate electronic atoms of signals from single-electron coherence. This procedure can be applied to data obtained from a numerical computation but also to experimental data obtained from an electronic tomography protocol as recently demonstrated in Ref. [9].

\section{FLOQUET-BLOCH-WANNIER ANALYSIS}

\section{A. Sketch of the method}

Equations (4), (6), and (8) have in common that their purely electron and purely hole parts are very simple. This characteristic is at the heart of our signal processing algorithm for analyzing single-electron coherence. The key idea, which is to exploit time periodicity of single-electron coherence

$$
\mathcal{G}_{\rho, x}^{(e)}\left(t+T \mid t^{\prime}+T\right)=\mathcal{G}_{\rho, x}^{(e)}\left(t \mid t^{\prime}\right),
$$

lies at the heart of Floquet theorem [52], the time-domain counterpart of Bloch's theorem for electronic waves in a periodic crystal [36].

However, in the present situation, we are looking for a simple description of $\mathcal{G}_{\rho}^{(e)}$ in terms of electron and hole excitations with respect to a reference Fermi sea (here $\left|F_{\mu=0}\right\rangle$ ). Consequently, Floquet's theorem has to be adapted in order to be compatible with the decomposition of the single-particle space of states into a direct sum $\mathcal{H}=\mathcal{H}_{+} \oplus \mathcal{H}_{-}$of electron and hole excitations that have positive (respectively, negative) energy with respect to the $\mu=0$ Fermi level. As we see, this can be done and the corresponding eigenvalues have a transparent physical meaning as an occupation number. Finally, as in band theory of solids, localized single-particle states [37] can then be constructed. We show in Sec. III C that these are the electronic atoms of signals suitable for describing the quantum electrical current under consideration.

\section{B. Floquet-Bloch analysis}

Introducing localized single-particle states $|t\rangle$ such that $\left\langle t \mid t^{\prime}\right\rangle=v_{F}^{-1} \delta\left(t-t^{\prime}\right)$, the dimensionless Hermitian operator $\mathbf{G}^{(e)}$ is defined by

$$
\mathbf{G}^{(e)}=v_{F}^{2} \int_{\mathbb{R}^{2}}|t\rangle \mathcal{G}^{(e)}\left(t, t^{\prime}\right)\left\langle t^{\prime}\right| d t d t^{\prime}
$$

in which the $(\rho, x)$ index is dropped out for simplicity. The conjugation relation $\mathcal{G}^{(e)}\left(t \mid t^{\prime}\right)^{*}=\mathcal{G}^{(e)}\left(t^{\prime} \mid t\right)$ for singleelectron coherence translates into $\mathbf{G}^{(e)}$ being Hermitian.
Furthermore, if we introduce the single-particle state $|\varphi\rangle$ corresponding to an excitation described by a normalized wave function $\varphi$

$$
|\varphi\rangle=v_{F} \int_{\mathbb{R}} \varphi(t)|t\rangle d t
$$

its occupation probability is a real number between 0 and 1 given by

$$
p[\varphi]=\left\langle\varphi\left|\mathbf{G}^{(e)}\right| \varphi\right\rangle
$$

thereby ensuring that $\mathbf{G}^{(e)}$ is a positive operator, bounded by 1 . For a $T$-periodic source, time periodicity of singleelectron coherence translates into the commutation of $\mathbf{G}^{(e)}$ with the time-translation operator $\mathbf{T}_{T}$ defined by $\mathbf{T}_{T}|t\rangle=$ $|t+T\rangle$.

As explained before, our analysis has to be performed separately on the electron and hole subspaces $\mathcal{H}_{ \pm}$. The adapted Floquet-Bloch theorem proven in Appendix B provides us with a basis of single-particle states, which partially diagonalizes the single-electron coherence operator while being compatible with the decomposition into electron and hole excitations.

More precisely, this result states that there exists an orthonormal basis $\left|\psi_{a, \nu}^{(e)}\right\rangle$ of the positive-energy Hilbert space $\mathcal{H}_{+}$and an orthonormal basis $\left|\psi_{b, v}^{(h)}\right\rangle$ of the negativeenergy Hilbert space $\mathcal{H}_{-}$, which are, respectively, indexed by band indexes $a$ (respectively, $b$ ) and quasienergies $0 \leq v<2 \pi f$, which are all eigenvectors of the timetranslation operator $\mathbf{T}_{T}$ with eigenvalue $e^{-i v T}$ and satisfy the normalization condition

$$
\left\langle\psi_{a, v}^{(e)} \mid \psi_{a^{\prime}, v^{\prime}}^{(e)}\right\rangle=2 \pi \delta_{a, a^{\prime}} \delta_{\mathbb{R} / 2 \pi f \mathbb{Z}}\left(v-v^{\prime}\right),
$$

where $\delta_{\mathbb{R} / 2 \pi f \mathbb{Z}}$ is a Dirac comb of period $2 \pi f$. A similar relation is obtained for the hole states $\left|\psi_{b, v}^{(h)}\right\rangle$. In these bases, the projections of the single-electron operators on the electron and hole quadrants (see Fig. 3) are diagonalized and their eigenvalues can be expressed as the occupation numbers of the corresponding single-electron states. Finally, the full electronic coherence $\mathbf{G}^{(e)}$ also contains the information on electron-hole coherences (see Fig. 3), which also commutes with $\mathbf{T}_{T}$. As explained in Appendix B, all this leads to the following form of single-electron coherence:

$$
\begin{aligned}
\mathbf{G}^{(e)}= & \int_{0}^{2 \pi f}\left\{\sum_{a} g_{a}^{(e)}(v)\left|\psi_{a, \nu}^{(e)}\right\rangle\left\langle\psi_{a, \nu}^{(e)}\right|\right. \\
& \left.+\sum_{b}\left[1-g_{b}^{(h)}(v)\right]\left|\psi_{b, \nu}^{(h)}\right\rangle\left\langle\psi_{b, v}^{(h)}\right|\right\} \frac{d v}{2 \pi}
\end{aligned}
$$




$$
\begin{aligned}
& +\sum_{a, b} \int_{0}^{2 \pi f}\left[g_{a b}^{(e h)}(v)\left|\psi_{a, \nu}^{(e)}\right\rangle\left\langle\psi_{b, v}^{(h)}\right|\right. \\
& \left.+g_{b a}^{(h e)}(v)\left|\psi_{a, \nu}^{(e)}\right\rangle\left\langle\psi_{b, v}^{(h)}\right|\right] \frac{d v}{2 \pi}
\end{aligned}
$$

where $g_{a b}^{(e h)}(v)=g_{b a}^{(h e)}(v)^{*}$ in order to ensure Hermiticity of $\mathbf{G}^{(e)}$. Let us note that the Floquet-Bloch wave functions being quasiperiodic, these are extended states, which are not localized on a specific period. This is not yet the description in terms of electronic atoms of signals that is discussed in the forthcoming subsection. Before moving to this description, let us recall that the outcome of the electronic tomography protocol originally proposed in Ref. [38] is an experimental determination of $\Delta_{0} \mathbf{G}^{(e)}$ to which the diagonalization procedure can be applied, therefore, leading to a Floquet-Bloch electronic and hole eigenstate and spectrum as was done in Ref. [9].

As discussed in Appendix B, the restriction $\mathbf{G}_{++}^{(e)}$ of the coherence operator $\mathbf{G}^{(e)}$ to the electron quadrant, is also positive and bounded by 1 . This leads to $0 \leq g_{a}^{(e)}(v) \leq 1$ for all $(a, v)$, thus showing that they can interpreted as the occupation number for the Floquet-Bloch electronic states $\left|\psi_{a, \nu}^{(e)}\right\rangle$. In the same way, $0 \leq g_{b}^{(h)}(v) \leq 1$ since $1-g_{b}^{(h)}(v)$ is the occupation number of the hole state $\left|\psi_{b, v}^{(h)}\right\rangle$.

\section{Electronic atoms of signal}

\section{Floquet-Wannier states}

Since we are interested in finding a description of the excess single-electron coherence in terms of electronic atoms of signal [29], which are normalized localized single-electron states, we consider Floquet-Wannier states, which are analogous to localized orbitals in solid-state band theory [37]. They are defined for $l \in \mathbb{Z}$ as

$$
\left|\varphi_{a, l}\right\rangle=\frac{1}{\sqrt{f}} \int_{0}^{2 \pi f} e^{-i v l t}\left|\psi_{a, \nu}\right\rangle \frac{d v}{2 \pi}
$$

and form an orthonormal family as implied by Eq. (14). Moreover, for a given band, all the states $\left(\left|\varphi_{a, l}\right\rangle\right)_{l \in \mathbb{Z}}$ are related by time translations since $\mathbf{T}_{T}\left|\psi_{a, \nu}\right\rangle=e^{-i \nu T}\left|\psi_{a, \nu}\right\rangle$ and Eq. (16) imply that

$$
\mathbf{T}_{T}\left|\varphi_{a, l}\right\rangle=\left|\varphi_{a, l+1}\right\rangle .
$$

Exactly as in solid-state band theory [53], there are ambiguities in the determination of electronic atoms of signals coming from the possibility to redefine the Floquet-Bloch eigenvectors at a given quasienergy $v$ within each degenerate eigenspace of the projection of $\mathbf{G}^{(e)}$ on the electron or the hole subspace. These ambiguities are extensively discussed in Appendix C. To circumvent these difficulties, we focus here on the electronic atoms of signal that have the smallest spreading in time. This minimal spreading principle [53], detailed in Appendix C2, has the advantage of producing maximally localized electronic atoms of signal. This provides a clear view of single-electron coherence within the electronic fluid in terms of single-particle states that are associated with a given period.

To understand the meaning of such a description, a musical analogy is convenient: the sound signal associated with a music instrument can be described in terms of elementary units, which are "music notes" arranged along a "music score," which specifies the notes to be emitted at a given time. The electronic atoms of signal can indeed be viewed as the electron quantum optics counterparts of "notes" and the expression of the excess single-electron coherence in the basis of "notes" can be viewed as its "quantum coherence score." We now discuss the specific form of the "quantum coherence score" of a $T$-periodic single-electron coherence.

\section{Quantum coherence score}

The single-electron coherence restricted to the electronic quadrant $\mathbf{G}_{++}^{(e)}$ can then be rewritten as

$$
\mathbf{G}_{++}^{(e)}=\sum_{a} \sum_{l_{+}, l_{-}} g_{a}^{(e)}\left(l_{+}-l_{-}\right)\left|\varphi_{a, l_{+}}^{(e)}\right\rangle\left\langle\varphi_{a, l_{-}}^{(e)}\right|,
$$

where

$$
g_{a}^{(e)}(l)=\int_{0}^{2 \pi f} g_{a}^{(e)}(v) e^{i v T l} \frac{d v}{2 \pi f}
$$

For $l \neq 0, g_{a}^{(e)}(l)$ represents the interperiod coherence over $|l|$ periods whereas $g_{a}^{(e)}(0)$ is the emission probability for the $\varphi_{a}$ electronic atom of signal at each period. Note that there is no coherence between electronic atoms of signals associated with different bands. However, electronic coherence may extend over more than one time period: a flat band $\left[g_{a}^{(e)}(v)\right.$ constant] will not lead to interperiod coherences whereas a nonflat band will. The typical scale over which $g_{a}^{(e)}(v)$ varies is nothing but the inverse time scale over which interperiod coherence exists. The same considerations apply to hole bands. Finally, using these Floquet-Wannier states, the electron-hole coherences $g_{a b}^{(e h)}\left(l_{+}-l_{-}\right)=\left\langle\varphi_{b, l_{-}}^{(h)}\left|\mathbf{G}^{(e)}\right| \varphi_{a, l_{+}}^{(e)}\right\rangle$ in this basis are given by

$$
g_{a b}^{(e h)}(l)=\int_{0}^{2 \pi f} g_{a b}^{(e h)}(v) e^{i l v T} \frac{d v}{2 \pi f} .
$$

Because electron-hole coherence couples different bands, different choices of electronic atoms of signal lead to different values for $g_{a b}^{(e h)}(\Delta l)$. This is not the case for the coherence between purely electronic or purely hole wave packets given by Eq. (19). 
Note that interperiod and electron-hole coherences make the "quantum coherence score" richer than an ordinary (classical) music score, which specifies only the note that has to be played at a given time and its intensity. The electronic atoms of signal and the associated "quantum coherence score" are the natural language to describe an arbitrary excess single-electron coherence. The "quantum coherence score" could in principle be used to encode some quantum information within a quantum electrical current.

Exactly as a tight-binding quadratic Hamiltonian in a specific Wannier orbital basis is a natural way to describe electron hopping within a condensed-matter system, the "quantum coherence score" is the first step in characterizing the many-body state of the electronic system. The excess second-order electronic coherence [28] can also be expressed in terms of electronic atoms of signal, thereby providing a view of the first nontrivial electronic correlations within the electronic fluid. Understanding the manybody state of the electronic fluid in terms of these discrete representations of first- and higher-order excess electronic coherences is a very interesting perspective for electron quantum optics. Although its simplest aspect is discussed in Sec. IV, a full discussion would go way beyond the scope of the present paper.

Positivity of the electronic and hole coherences $\mathbf{G}^{(e)}$ and $\mathbf{G}^{(h)}$ leads to Cauchy-Schwarz inequalities. Within the electron and hole quadrants, it leads to

$$
\begin{aligned}
& \left|g_{a}^{(e)}(l)\right| \leq \min \left[g_{a}^{(e)}, 1-g_{a}^{(e)}\right], \\
& \left|g_{b}^{(h)}(l)\right| \leq \min \left[g_{b}^{(h)}, 1-g_{b}^{(h)}\right],
\end{aligned}
$$

with $g_{a}^{(e)}=g_{a}^{(e)}(l=0)$ and $g_{b}^{(h)}=g_{b}^{(h)}(l=0)$ denoting the respective averages of $g_{a}^{(e)}(v)$ and $g_{b}^{(h)}(v)$ over $0 \leq v<$ $2 \pi f$. In the electron-hole quadrants, the Cauchy-Schwarz inequalities bound the electron-hole coherences:

$$
\begin{aligned}
& \left|g_{a b}^{(e h)}(l)\right|^{2} \leq g_{a}^{(e)}\left[1-g_{b}^{(h)}\right], \\
& \left|g_{a b}^{(e h)}(l)\right|^{2} \leq g_{b}^{(h)}\left[1-g_{a}^{(e)}\right] .
\end{aligned}
$$

These inequalities immediately show that, in the absence of electron $\left[g_{a}^{(e)}(v)=0\right.$ for all $v$ and $\left.a\right]$ or hole $\left[g_{b}^{(h)}(v)=\right.$ $0]$ excitations, there are no electron-hole coherences $\left[g_{a b}^{(e h)}(l)=0\right]$ as well as no coherence between the missing excitations as noted in Ref. [40].

\section{Martin-Landauer wave packets}

Let us illustrate these ideas on the example of a stationary electronic state. In this case, the single-electron coherence only depends on $t-t^{\prime}$ and is the Fourier transform of the electronic distribution function $f_{e}(\omega)$. Such a state can be viewed as $T$ periodic for any period $T$ so, let us choose one and perform the corresponding Floquet-Bloch analysis. The excess single-electron coherence being already diagonal in the plane-wave basis, the Floquet-Bloch waves are plane waves $\psi_{m, v}(t)=v_{F}^{-1 / 2} e^{-i(v+2 \pi m f) t}(0 \leq$ $v<2 \pi f)$. The bands are then indexed by an integer $m \in$ $\mathbb{N}$ and the corresponding eigenvalues are given by

$$
g_{m}^{(e)}(v)=f_{e}(v+2 \pi m f)
$$

The corresponding electronic atoms of signal are obtained by summing plane waves over an energy band of width $h f$, centered at energies $(m+1 / 2) h f$ with $m$ integer. These are the Martin-Landauer wave packets [41]:

$$
\varphi_{\mathrm{ML}_{m, 0}}(t)=\frac{1}{\sqrt{v_{F} T}} \frac{\sin (\pi f t)}{\pi f t} e^{-2 i \pi(m+1 / 2) f t},
$$

which are known in the signal-processing community as the Shannon wavelets. Their Wigner representation $W_{\mathrm{ML}_{m, l}}(t, \omega)=W[t-l T, \omega-2 \pi(m+1 / 2) f]$, defined for a single-electron wave packet $\varphi$ by using $\varphi(t+\tau / 2) \varphi(t-$ $\tau / 2)^{*}$ instead of $\mathcal{G}^{(e)}(t+\tau / 2, t-\tau / 2)$ in Eq. (2), are time and frequency translated from

$$
W(t, \omega)=\mathrm{H}[\pi f-|\omega|]\left(1-\frac{|\omega|}{\pi f}\right) \operatorname{sinc}[2(\pi f-|\omega|) t] .
$$

where $\mathrm{H}$ is the Heaviside step function. The Wigner representation $W_{\mathrm{ML}_{0,0}}$, depicted on Fig. 4, is clearly localized in the $0 \leq \omega \leq 2 \pi f$ energy band and decays as $1 / t$ in time.

Because of Eq. (23), the bands are generically not flat thereby implying the existence of interperiod coherences and, therefore, of an associated coherence time. The idea is then to choose the period $T$ so that, the electron distribution function is as flat as possible over energy bands of width $h f$.

For example, at zero temperature, the nonequilibrium distribution function generated by a dc biased QPC with $V_{\mathrm{dc}}<0$, is a step function that jumps from 1 for $\omega<0$

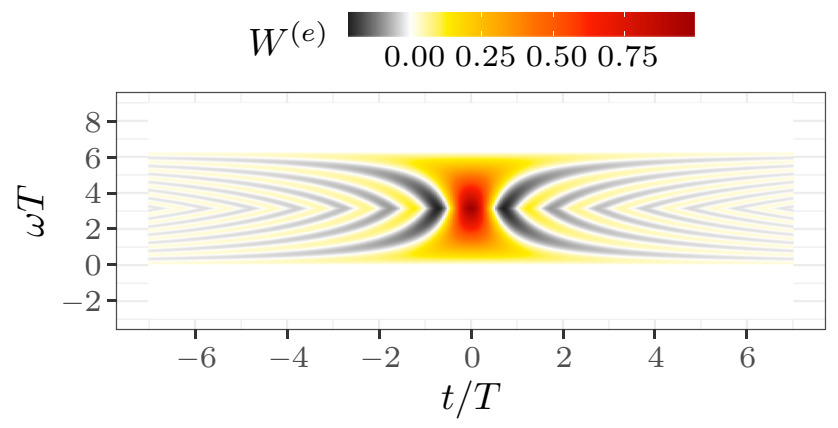

FIG. 4. Wigner representation of the Martin-Landauer wave packet $\mathrm{ML}_{0,0}$ as a function of $t / T$ and $\omega T$. 
to the transmission probability $0<\mathcal{T}<1$ from the biased incoming electrode to the outgoing one we are considering for $0 \leq \omega \leq-e V_{\mathrm{dc}} / \hbar$. It then abruptly falls to zero $\omega>-e V_{\mathrm{dc}} / \hbar>0$. The natural choice of $T$ is then $T=$ $h / e\left|V_{\mathrm{dc}}\right|$. The excess coherence has only one nontrivial band corresponding to $g_{0}^{(e)}(v)=\mathcal{T}$ for $0 \leq v<2 \pi f=$ $\left|e V_{\mathrm{dc}}\right| / \hbar$. The excess electronic coherence is then naturally described in terms of Martin-Landauer wave packets associated with period $T=h / e\left|V_{\mathrm{dc}}\right|$ :

$$
\Delta_{0} \mathbf{G}^{(e)}=\mathcal{T} \sum_{l \in \mathbb{Z}}\left|\mathrm{ML}_{0, l}\right\rangle\left\langle\mathrm{ML}_{0, l}\right| .
$$

Consequently, this excess single-electron coherence corresponds to a train of Martin-Landauer wave packets without interperiod coherences and each of them being emitted with probability $\mathcal{T}$. Stationarity is visible through the invariance of $\Delta_{0} \mathbf{G}^{(e)}$ through translation by $\mathbf{T}_{\Delta t}$ for any $\Delta t$ : time shifting all the Martin-Landauer wave packets in the rhs of Eq. (26) $\left|\mathrm{ML}_{m, l}\right\rangle \mapsto \mathbf{T}_{\Delta t}\left|\mathrm{ML}_{m, l}\right\rangle$ still gives $\Delta_{0} \mathbf{G}^{(e)}$.

At finite temperature $T_{\mathrm{el}}>0 \mathrm{~K}$, the electronic distribution function is smeared over a scale $k_{B} T_{\mathrm{el}} / \hbar$, thus leading to a nonflat band spectrum. Therefore, there are always interperiod coherences over the thermal coherence time $h / k_{B} T_{\mathrm{el}}$. It might seem surprising that when $T_{\mathrm{el}}=0 \mathrm{~K}$, the interperiod coherences go to zero whereas the thermal coherence time goes to infinity. This comes from the fact that when decreasing the temperature, as the offdiagonal coherences spread over more and more period, their modulus decreases and ultimately vanishes at zero temperature.

\section{Relation to experimentally relevant quantities}

Let us now explain how experimental signals are related to these spectral quantities. We first discuss the value of the dip in an HOM experiment, a simple HOM-based repeated-detection scheme of a given electronic excitation and finally a time-dependent energy filter based on a driven QPC [54].

\section{The Hong-Ou-Mandel dip}

In the case of an HOM experiment with two identical sources $S_{1}$ and $S_{2}$ on the incoming channels of a beam splitter with reflection and transmission probabilities $\mathcal{R}$ and $\mathcal{T}$, the depth of the HOM dip, obtained by synchronizing the sources, can be related to the Floquet-Bloch spectral properties of single-electron coherence. This comes from the expression of the two-particle interference contribution to low-frequency noise in an HOM experiment as [38]

$$
\mathcal{Q}\left(t, t^{\prime}\right)=-e^{2} v_{F}^{2} \mathcal{R} \mathcal{T}\left(\mathcal{G}_{1}^{(e)}\left(t, t^{\prime}\right) \mathcal{G}_{2}^{(h)}\left(t, t^{\prime}\right)+[1 \leftrightarrow 2]\right) .
$$

We consider the low-frequency noise defined by integrating over $\tau=t-t^{\prime}$ and averaging over $\bar{t}=\left(t+t^{\prime}\right) / 2$.
Expanding both contributions in the rhs of Eq. (27) in terms of $\Delta_{0} \mathbf{G}^{(e)}$ leads to three distinct contributions. Two of them involve only one of the incoming excess singleelectron coherences and correspond to the partitioning of single-particle excitations from one of the two incoming channels at the QPC [Hanbury Brown and Twiss (HBT) contribution] whereas the third one involves the excess single-electron coherence of both sources and accounts for two-particle interferences between them (HOM contribution).

At zero temperature and with identical and synchronized sources on two incoming channels the excess noise in the HBT (only one source on) and HOM experiments (both sources on) are obtained as sums of a background, which comes from the transmitted or reflected excess noise $\Delta S_{S}$ of the sources and of two-excitation interference contributions denoted by $\Delta S_{\mathrm{HBT}}$ and $\Delta S_{\mathrm{HOM}}$ :

$$
\begin{aligned}
\Delta S_{11}^{\left(\mathrm{HBT}_{1}\right)} & =\mathcal{R}^{2} \Delta S_{\mathrm{S}}+\Delta S_{\mathrm{HBT}}, \\
\Delta S_{11}^{\left(\mathrm{HBT}_{2}\right)} & =\mathcal{T}^{2} \Delta S_{\mathrm{S}}+\Delta S_{\mathrm{HBT}}, \\
\Delta S_{11}^{(\mathrm{HOM})} & =\left(\mathcal{R}^{2}+\mathcal{T}^{2}\right) \Delta S_{S}+\Delta S_{\mathrm{HOM}} .
\end{aligned}
$$

As shown in Appendix D, $\Delta S_{\mathrm{HBT}}$ and $\Delta S_{\mathrm{HOM}}$ are given by

$$
\Delta S_{\mathrm{HBT}}=e^{2} \mathcal{R} \mathcal{T} \int_{0}^{2 \pi f}\left[\sum_{a} g_{a}^{(e)}(v)+\sum_{b} g_{b}^{(h)}(v)\right] \frac{d \nu}{2 \pi},
$$

$$
\begin{aligned}
\Delta S_{\mathrm{HOM}}= & 2 e^{2} \mathcal{R} \mathcal{T}\left\{\int_{0}^{2 \pi f} \sum_{a}\left[1-g_{a}^{(e)}(v)\right] g_{a}^{(e)}(v) \frac{d \nu}{2 \pi}\right. \\
& +\int_{0}^{2 \pi f} \sum_{b} g_{b}^{(h)}(v)\left[1-g_{b}^{(h)}(v)\right] \frac{d v}{2 \pi} \\
& \left.-2 \int_{0}^{2 \pi f} \sum_{a, b}\left|g_{a b}^{(e h)}(v)\right|^{2} \frac{d v}{2 \pi}\right\}
\end{aligned}
$$

In Appendix D 2, we show that the depth of the HOM dip, which is the difference $\Delta S_{\mathrm{dip}}=2 \Delta S_{\mathrm{HBT}}-\Delta S_{\mathrm{HOM}}$ at this operating point can be expressed simply in terms of the fluctuation of the total charge emitted per period $(\Delta Q)_{W}^{2}$ :

$$
\frac{\left[\Delta S_{\mathrm{dip}}\right]}{\left[\Delta S_{\mathrm{dip}}^{(\max )}\right]}=1-\frac{(\Delta Q)_{W}^{2}}{\bar{N}_{\text {tot }}}
$$

in which $\bar{N}_{\text {tot }}$ is the sum of the average number of electron and hole excitations [see Eq. (D11)] and $(\Delta Q)_{W}^{2}$ is computed from first-order coherences using Wick's theorem (see Appendix D 2). If the many-body state does satisfy Wick's theorem, which is the case whenever interactions 
can be neglected, then a maximal dip depth corresponds to the actual vanishing of charge fluctuations. Under this hypothesis, an ideal single-electron [see Eq. (4)] or singleelectron and single-hole [see Eq. (6)] source would reach this bound and, therefore, under the assumption that interactions can be neglected, would lead to a maximally deep HOM dip at zero temperature. Another important example is the state with a single coherent electron-hole pair obtained by the action of an operator of the form

$$
\sqrt{1-g} \mathbf{1}+e^{i \Theta} \sqrt{g} \psi^{\dagger}\left[\varphi_{e}\right] \psi\left[\varphi_{h}\right]
$$

where $0<g<1$, acting on the Fermi sea $\left|F_{\mu=0}\right\rangle$. This state belongs to the null eigenstate of the excess charge operator (with respect to $\left|F_{\mu=0}\right\rangle$ ). This explains that the excess charge fluctuation $(\Delta Q)_{w}^{2}$ as well as the average excess charge is exactly zero. But it is not an eigenstate of the electron (or hole) excitation number operators: the average number of electron (as well as hole) excitations is $g$ and its fluctuation $g(1-g)$. It follows from Eq. (30) that the dip reaches its maximum value. As we see in Sec. $\mathrm{IV}$, this is the case for all states obtained by acting with a $T$-periodic time-dependent scatterer on $\left|F_{\mu=0}\right\rangle$.

However, let us recall that this is not true when interactions, for example between the sources and the beam splitter are present [50]: the depth of the dip is decreased by electronic decoherence. The dip may also not be maximally deep, at zero temperature, when the emission process involves some classical randomness, one example being the randomized train of levitons considered in Sec. V B 2.

\section{Repeated HOM detections}

Because electronic atoms of signals are localized in time, they are suitable single-particle states to discuss repeated detection protocols. Let us discuss such a protocol based on two-particle interferometry using an ideal beam splitter with energy-independent transmission probability $\mathcal{T}$ (HOM interferometry).

On one incoming channel, we consider a $T$-periodic source $S$ whereas on the other incoming channel, we have a specific ideal electronic source $S_{a}$, which emits a periodic train of electronic atoms of signals $\left|\varphi_{a, l}\right\rangle$, not necessarily related to the ones emitted by $S$. Its excess single-electron coherence is thus

$$
\Delta \mathcal{G}_{S_{a}}^{(e)}\left(t, t^{\prime}\right)=\sum_{l=0}^{N} \varphi_{a, l}(t) \varphi_{a, l}^{*}\left(t^{\prime}\right)
$$

The resulting outgoing current noise contains a HOM contribution proportional to the overlap between $\Delta \mathcal{G}_{S}^{(e)}$ and $\Delta \mathcal{G}_{S_{a}}^{(e)}$ [40]. Using the $T$ periodicity of $\Delta \mathcal{G}_{S}^{(e)}$, the experimental signal scales as $N \gg 1$, which quantifies the total acquisition time $N T$ :

$$
\int_{[-N T / 2, N T / 2]^{2}} \Delta \mathcal{G}_{S_{a}}^{(e)}\left(t, t^{\prime}\right)^{*} \Delta \mathcal{G}_{S}^{(e)}\left(t, t^{\prime}\right) d t d t^{\prime} \sim N \bar{p}_{a} .
$$

This overlap counts the number of times an electron in the single-particle state $\left|\varphi_{a, l}\right\rangle$ is scattered against an electronic excitation in the same single-particle state for $N$ periods of duration $T$. Since $S_{a}$ is an ideal source sending a train of $N$ identical excitations shifted by multiples of $T$, the quantity $\bar{p}_{a}$ should be interpreted as the average number of times, the single-particle state $\varphi_{a}$ is emitted per period. If the $\varphi_{a, l}$ are among the electronic atoms of signal emitted by $S$, then when the emission of $S_{a}$ is synchronized with the emission of these atoms of signal by $S, \bar{p}_{a}=g_{a}^{(e)}$ is the probability of emission of these electronic atoms of signal by $S$.

\section{Time-frequency filtering}

A repeated detection protocol can also be realized by scattering the electron flow through a periodically driven energy-dependent scatterer. Recently, such a timefrequency filtering has been demonstrated and used for single-electron tomography [35]. It relies on a quantum point contact with an energy-dependent transmission probability $T(\omega)$ equipped with a top electrostatic gate driven by a time-dependent voltage $V_{d}(t)$. The signal collected by such a device is the total charge transmitted through the QPC, which can be rewritten as a linear filtering of the incident single-electron excess coherence [54]:

$$
Q=-e \int_{\mathbb{R}^{2}} v_{F} \Delta \mathcal{G}^{(e)}\left(t+\frac{\tau}{2}, t-\frac{\tau}{2}\right) \mathcal{F}_{d}(t, \tau)^{*} d t d \tau,
$$

with the filter's kernel being given by

$$
\mathcal{F}_{d}(t, \tau)=\int_{\mathbb{R}} T(\omega) e^{i \omega \tau+(i e / \hbar) \int_{t-\tau / 2}^{t+\tau / 2} \mathcal{V}_{d}\left(t^{\prime}\right) d t^{\prime}} \frac{d \omega}{2 \pi}
$$

in which $\mathcal{V}_{d}\left(t^{\prime}\right)$ is proportional to the driving voltage $V_{d}$ [55].

For a time-periodic driving at frequency $f=1 / T$, the linear filter is also $T$ periodic. Since $\mathcal{F}_{d}(t, \tau)^{*}=\mathcal{F}_{d}(t,-\tau)$, the Floquet-Bloch analysis can be applied to the filter. Besides the example considered in Ref. [54], the case of a driven quantum dot $[56,57]$ corresponding to a Lorentzian transmission probability $T(\omega)$ centered at $\omega_{0}>0$ with width $\gamma_{0} \ll \omega_{0}$ is worth considering since it corresponds to a dot filtering mostly electronic excitations around the energy $\hbar \omega_{0}$. Provided the drive is such that $\omega_{0}-$ $e \mathcal{V}_{d}(t) / \hbar \gg \gamma_{0}$, we expect that only purely electronic excitations are transmitted. We should, therefore, be able to 
diagonalize the (dimensionless) filtering operator

$$
\mathbf{F}_{d}=v_{F} \int_{\mathbb{R}^{2}}\left|t_{+}\right\rangle \mathcal{F}_{d}\left(\frac{t_{+}+t_{-}}{2}, t_{+}-t_{-}\right)\left\langle t_{-}\right| d t_{+} d t_{-}
$$

within the electronic quadrant, thus leading to

$$
\mathbf{F}_{d}=\sum_{\alpha} \int_{0}^{2 \pi f} \mathcal{F}_{\alpha}(\nu)\left|\psi_{\alpha, \nu}^{(d)}\right\rangle\left\langle\psi_{\alpha, \nu}^{(d)}\right| \frac{d \nu}{2 \pi}
$$

in which the eigenstates $\left|\psi_{\alpha, \nu}^{(d)}\right\rangle$ are electronic FloquetBloch waves of the filter. The eigenvalues $\mathcal{F}_{\alpha}(v)$ are real but (as far as we know) are not restricted. In practice, acquisition of the experimental signal requires a $T$-periodic single-electron excess coherence $\Delta_{0} \mathbf{G}^{(e)}$ and measurement over $N \gg 1$ periods. Then, the total transmitted charge increases linearily with time. The average charge transmitted per period $Q_{T}$, and thereby the transmitted dc current $\left\langle I_{\mathrm{tdc}}\right\rangle=Q_{T} / T$, can then be expressed in terms of the electronic atoms of signals $\left|\varphi_{\alpha, l}^{(d)}\right\rangle$ arising from the Floquet-Bloch waves of the filter. Decomposing

$$
\mathbf{F}_{d}=\sum_{\alpha} \sum_{\left(l_{+}, l_{-}\right) \in \mathbb{Z}^{2}} \mathcal{F}_{\alpha}\left(l-l^{\prime}\right)\left|\varphi_{\alpha, l_{+}}^{(e)}\right\rangle\left\langle\varphi_{\alpha, l_{-}}^{(e)}\right|
$$

in which $\mathcal{F}_{\alpha}\left(l_{+}-l_{-}\right)$is related to $\mathcal{F}_{\alpha}(v)$ by Eq. (19), leads to the average transmitted dc current:

$$
\left\langle I_{\mathrm{tdc}}\right\rangle=-e f \sum_{\alpha} \sum_{l \in \mathbb{Z}} \mathcal{F}_{\alpha}(l)\left\langle\varphi_{\alpha, 0}^{(d)}\left|\Delta_{0} \mathbf{G}^{(e)}\right| \varphi_{\alpha, l}^{(d)}\right\rangle .
$$

Therefore, the driven QPC studied in Ref. [54] appears as a linear filter acting on linear coherence, which generalizes to the time-dependent case, the quantum-dot energy filter originally used to study electronic relaxation in quantum Hall edge channels [58]. The average current is then directly proportional to the overlap between $\Delta_{0} \mathbf{G}^{(e)}$ and the time-dependent filter's "quantum coherence score" introduced in Sec. III C. Generically, several bands may be present and, therefore, several electronic atoms of signal may be needed. However, we can hope that suitable drives may lead to filtering by mostly one band, or equivalently one type of electronic atom of signal thereby enabling us to probe the presence of a specific atom of signal within $\Delta_{0} \mathbf{G}^{(e)}$.

\section{MANY-BODY PROPERTIES}

Until now, we have focused on the properties of the electronic fluid at the single-particle level, assuming nothing more than $T$ periodicity. However, when interactions within the electronic fluid can be neglected, the singleparticle description actually gives us access to the whole many-body state. This is notably the case when the singleelectron source is modeled by single-particle scattering processes.
In this section, we explain how the Floquet-Bloch analysis allows us to give a simple many-body description and unravel some of the symmetries hidden within the band structure. We are also able to rederive the single-particle scattering operator leading to such a single-electron coherence, thereby exploring the path followed in Ref. [44] the other way around. Furthermore, by giving a direct insight on electron-hole entanglement, the Floquet-Bloch analysis is well suited to quantify the quality of electron sources. We use it in Sec. V to identify the best operating experimental parameters for a given source.

\section{A. Many-body state at zero temperature}

For the sake of simplicity but without loss of generality, we focus on a $T$-periodic coherence corresponding to a vanishing average dc current so that the chemical potential of the electron fluid is exactly zero, a specific case also considered in Ref. [59]. We assume that single-electron coherence is the result of $T$-periodic single-particle scattering $\mu=0$ Fermi sea $\left(T_{\mathrm{el}}=0 \mathrm{~K}\right)|F\rangle$, which thereby generally describes a $T$-periodic ac source whenever interactions can be neglected.

In order to derive the many-body state at zero temperature, the method consists of finding an expression of the many-body Floquet operator from the Floquet-Bloch decomposition (see Appendix E) thereby inverting the procedure described in Refs. [44,59]. Applying this operator to the Fermi sea leads to the general form of many-body state $|\Psi\rangle$ emitted by the source:

$$
\prod_{\substack{v \in[0,2 \pi f[ \\a \in \mathbb{N}}}\left\{\sqrt{1-g_{a}^{(e)}(v)}+\sqrt{g_{a}^{(e)}(v)} \psi^{\dagger}\left[\psi_{a, \nu}^{(e)}\right] \psi\left[\psi_{a, \nu}^{(h)}\right]\right\}|F\rangle .
$$

From this expression, we notice an important symmetry on the spectrum: because electrons and holes are emitted in pairs, we have $g_{a}^{(e)}(v)=g_{a}^{(h)}(v)$. It is worth noting that this relation is different from electron-hole symmetry, which reverses frequencies and, as such, would be $g_{a}^{(e)}(v)=$ $g_{a}^{(h)}(2 \pi f-v)$. Consequently, when electron-hole symmetry is satisfied in state (40), the Floquet-Bloch spectrum exhibits the symmetry: $g_{a}^{(e)}(\nu)=g_{a}^{(e)}(2 \pi f-v)$.

\section{The case of flat bands}

When the bands are flat, we can go further in the analysis and re-express the many-body state in terms of FloquetWannier wave functions. The flat band case $\left[g_{a}(v)=g_{a}\right]$ happens for a purely ac voltage drive at zero temperature. The case of ac voltage drives have been studied previously in Refs. [60-62]. In this case, as shown in Appendix E, 
Eq. (40) can be rewritten as

$$
|\Psi\rangle=\prod_{a \in \mathbb{N}} \prod_{l \in \mathbb{Z}}\left\{\sqrt{1-g_{a}}+\sqrt{g_{a}} \psi^{\dagger}\left[\varphi_{a, l}^{(e)}\right] \psi\left[\varphi_{a, l}^{(h)}\right]\right\}|F\rangle .
$$

This is the formula for a classical voltage drive found in Ref. [61]. Since there is no relative phase between $\sqrt{1-g_{a}}$ and $\sqrt{g_{a}} \psi^{\dagger}\left[\varphi_{a, l}^{(e)}\right] \psi\left[\varphi_{a, l}^{(h)}\right]$, once a determination for the electronic Floquet-Wannier wave functions has been chosen, it determines also the wave functions for holes, up to a global phase. As such, we do not expect the Floquet-Wannier wave functions to be minimally spread for both electrons and holes. This also allows us to come back to the ansatz guessed in Eq. (8). This ansatz works only in the case of flat bands. While this is the case for a classical drive, we see that it is usually not the case for the mesoscopic capacitor.

\section{B. Electron-hole entanglement entropy}

Accessing the many-body state allows us to quantify the quality of a single-electron (or more generally, a $n$ electron) source. Such a source would emit electrons and holes independently, without correlations besides Fermi statistics. Furthermore, because our sources are described as noiseless single-particle scattering from an equilibrium state, quantifying outgoing correlations gives the amount of correlation generated during the scattering process. When the global state is pure $\left(T_{\mathrm{el}}=0 \mathrm{~K}\right)$, the correlations only come from entanglement.

Although the question of entanglement is a complicated problem in a many-body system [63], the very definition of electron and hole provides us with a natural way to split the many-body Hilbert space in two orthogonal components, thereby enabling us to fall back on a more familiar description. Thanks to the parity superselection rule for fermions [22-24] and to the absence of superconducting correlations in a metallic conductor, the many-body density operator is block diagonal, only exhibiting coherences between states having the same number of electron and hole excitations with respect to a reference Fermi sea. Quantifying the electron-hole entanglement could, in principle, be done by looking separately into all these superselection sectors but this would require knowing electronic coherences to all orders.

Fortunately, when Wick's theorem is satisfied, the full many-body state depends only on first-order coherence. This is also true for the partial trace on positive or negative energy states since higher-order correlations functions expressed in frequency basis are just correlation functions of the whole state taken in the simplex of positive frequencies and thereby, they also obey Wick's theorem. From this and the superselection rule follows that the many-body state associated to the electronic quadrant corresponds to filling noncoherently each Floquet-Bloch mode $\left|\psi_{a, v}^{(e)}\right\rangle$ with its probability $g_{a}^{(e)}(v)$ :

$$
\begin{aligned}
\widehat{\rho}_{++}= & \bigotimes_{\substack{v \in[0,2 \pi f] \\
a \in \mathbb{N}}}\left\{\left[1-g_{a}^{(e)}(v)\right]|0\rangle\langle 0|\right. \\
& \left.+g_{a}^{(e)}(v) \psi^{\dagger}\left[\psi_{a, \nu}^{(e)}\right]|0\rangle\langle 0| \psi\left[\psi_{a, \nu}^{(e)}\right]\right\} .
\end{aligned}
$$

Its form is reminiscent of a the thermal state with a modedependent temperature.

An important property of an ideal $n$-electron source is that there are no correlations between the electron and hole excitations it emits. Namely, we expect the full many-body state associated to positive and negative frequencies to factorize as $\widehat{\rho}_{\mathrm{SES}}=\widehat{\rho}_{++} \otimes \widehat{\rho}_{--}$. In the present case of a pure many-body state for the whole electronic fluid, the departure from such a factorized form is measured by the von Neumann entanglement entropy of the electrons (or the holes), a quantitative measure of entanglement in this case [64]. Starting from Eq. (42) for the many-body state, it is given by

$$
\begin{aligned}
S_{\mathrm{VN}}=- & \sum_{a \in \mathbb{N}} \int_{0}^{2 \pi f}\left(g_{a}^{(e)}(v) \log _{2}\left[g_{a}^{(e)}(v)\right]\right. \\
& \left.+\left[1-g_{a}^{(e)}(v)\right] \log _{2}\left[1-g_{a}^{(e)}(v)\right]\right) \frac{d v}{2 \pi f} .
\end{aligned}
$$

Therefore, the entanglement entropy can be inferred from the properties of the Floquet-Bloch spectrum for electrons (or holes), which thereby appears as an entanglement spectrum [65]. This connection has been exploited to quantify entanglement between spatially separated regions in manybody fermionic systems or generated by a quantum point contact [66] but can indeed be applied to more general decompositions of the full single-particle state in a sum of two orthogonal components [67].

Finally, at nonzero temperature, the von Neumann entropy is no longer the sole measure of entanglement since the global state is not pure any more. For practical use in experiments [9], the departure of the full many-body state from a pure state can be quantified using a purity indicator, which can also be expressed in terms of the Floquet-Bloch electron and hole spectra $\left[g_{a}^{(e)}(v), g_{b}^{(h)}(v)\right]$ and of electron hole coherences $g_{a b}^{(e h)}(v)$ for $0 \leq v<2 \pi f$ as detailed in Appendix F.

\section{ELECTRON SOURCE ANALYSIS}

We now apply our signal-processing technique to numerical data coming from a Floquet modelization of periodic electron sources. Our goal is to use the FloquetBloch spectrum to assess the quality of sources as singleelectron sources, along the lines discussed in the previous section. Because of their experimental importance, the mesoscopic capacitor [1] and the Leviton source $[3,68]$ 
are discussed. The former offers the possibility to emit single-electron excitations well separated from the Fermi surface. The Leviton source exploits the fact that a suitable rearrangement of an infinite Fermi sea can lead to the generation of purely electronic excitations [69] in a simple way.

\section{A. The mesoscopic capacitor}

\section{Model}

The mesoscopic capacitor depicted on Fig. 2 is modeled using Floquet scattering theory [70], since, in most experimentally relevant regimes, interaction effects within the capacitor itself can be neglected. Within this framework [38,71], the mesoscopic capacitor is characterized by the level spacing of the dot $\Delta$, the transparency $D$ of the QPC (see Fig. 2) as well as by the voltage drive $V_{g}(t)$. The Floquet scattering matrix relating the outgoing fermionic field to the incoming one is then expressed as $[38,71]$

$$
S\left(t, t^{\prime}\right)=\exp \left[\frac{i e}{\hbar} \int_{t^{\prime}}^{t} V_{g}(\tau) d \tau\right] \mathcal{S}_{0}\left(t-t^{\prime}\right)
$$

where $\mathcal{S}_{0}$ denotes the scattering matrix of the dot, which, in the frequency domain, is given by

$$
\mathcal{S}_{0}(\omega)=\frac{\sqrt{1-D}-e^{2 i \pi \hbar\left(\omega-\omega_{0}\right) / \Delta}}{1-\sqrt{1-D} e^{2 i \pi \hbar\left(\omega-\omega_{0}\right) / \Delta}},
$$

where $\omega_{0}$ comes from a dc bias applied to the dot. Adjusting it so that $\omega_{0}=0$ ensures that a peak in the density of states of the dot is located at the Fermi level in the absence of external drive.

We now discuss the operating regimes of the mesoscopic capacitor operated by a sinusoidal drive $V_{g}(t)=$ $V \sin (2 \pi f t)$ at frequency $f$ by computing the electron and hole entanglement from the Floquet-Bloch spectrum for the electronic excitations at fixed $\Delta$ and driving frequency $f$ in terms of the experimentally controlled parameters $D$ and $V$, the latter being the amplitude of the drive applied to the mesoscopic capacitor. In the present case, we work at fixed drive frequency and dot geometry so that $\Delta / h f \simeq$ 20 , which corresponds to experimentally realistic conditions. Having in mind the original experiments [1,72], results for a square drive are given in Appendix G.

\section{Electron-hole entanglement entropy}

Figure 5 presents a density plot of the entropy defined by Eq. (43) as a function of $D$ and $e V / \Delta$ at fixed $\Delta / h f=$ 20. There are shallow zones with minima in each square $e V / \Delta \in] n, n+1](n \in \mathbb{N})$ and $0<D \leq 1$. A global minimum can be found at $e V_{\text {opt }} / \Delta$ slightly less than 0.24 and $D_{\text {opt }} \approx 0.38$ and the corresponding entropy is very low: 0.06 bit. This is a regime where the mesoscopic capacitor behaves almost ideally, emitting exactly one electron

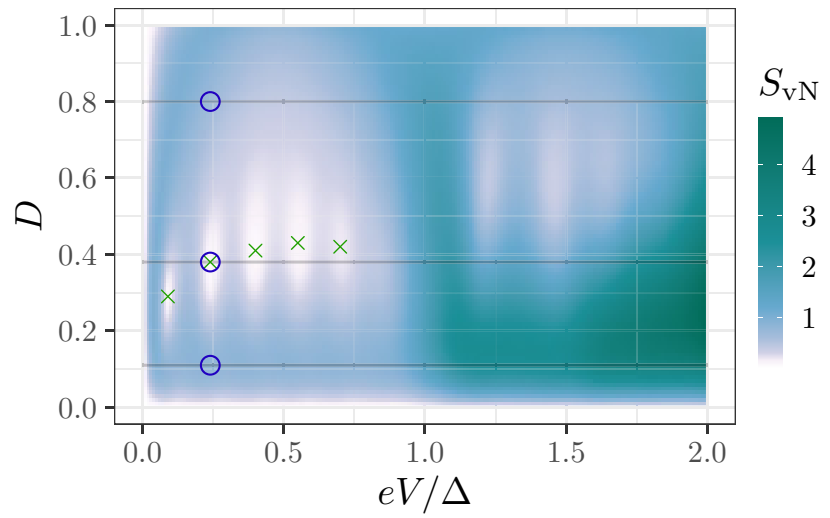

FIG. 5. Density plot of the electron-hole entanglement entropy at zero temperature for the mesoscopic capacitor operated with a sine drive at frequency $f$ such that $\Delta / h f=20$ as a function of $\mathrm{eV} / \Delta$ and $D$. Crosses correspond to the five local minima of the entropy (see Table I), where the source is the closest to an ideal single-electron source. For the second local minimum, we choose three values of $D$ : the optimal one, one above, and one below (round points) to discuss the effect of varying $D$ for a fixed drive.

and one hole excitation per period. Decreasing $D$ from this value leads to a local maximum of the entropy (for $D \simeq 0.11$ ) before a decrease to zero when $D \rightarrow 0$, corresponding to a regime where the source emits nothing. For each of the three points located at the same value of $\mathrm{eV} / \Delta$ and corresponding to $D=0.8, D=D_{\text {opt }}$, and $D \simeq 0.11$, the full electronic Wigner distribution function is depicted on Fig. 6. As for the square drive case, interference fringes, characteristic from interperiod coherences as well as for electron-hole coherences, are visible for $D \simeq 0.11$ and to a lesser extent for $D=0.8$ whereas they are much more discrete for $D=D_{\mathrm{opt}}$.

The local minima on Fig. 5 correspond to quite low values of the electron-hole entanglement entropy. They can also be seen on Fig. 7 presenting cuts for a fixed value of

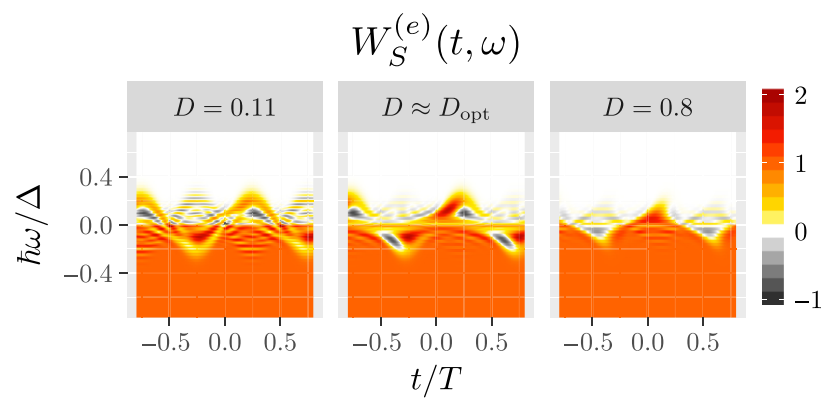

FIG. 6. Density plots of the full Wigner distribution function $W_{S}^{(e)}(t, \omega)$ for the sine-drive case as a function of $t / T$ and $\hbar \omega / \Delta$ for the three round points appearing on Fig. 5. 


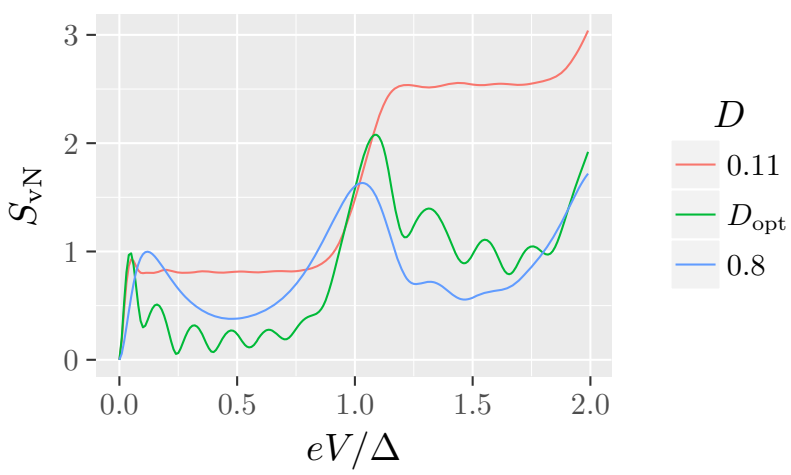

FIG. 7. Cuts of the entropy $S_{\mathrm{vN}}$ in the sine-drive case for the three horizontal lines corresponding to $D=0.11, D=D_{\text {opt }}$, and $D=0.8$ on Fig. 5 as functions of $e V / \Delta$.

$D$ of $S_{\mathrm{vN}}$ as functions of $e V / \Delta$. By running a simplex minimization algorithm, we can find position and entropy value at each minimum as summarized on Table I.

There are also local minima in the second square where $1<e V / \Delta \leq 2$ but the corresponding entropy values are higher (above $0.3 \mathrm{bit}$ ). In this zone, we send three electrons and three holes per period. As such, it is not surprising that the purity of the source is lower, since we expect to excite more electron-hole pairs.

\section{The Floquet-Bloch spectrum}

Let us review the Floquet-Bloch spectra for the three round points marked on Fig. 5. The middle and right panels of Fig. 8 depict flat bands. The middle panel corresponds to the absolute minimum of the entropy, showing one band with an average very close to 1 . This corresponds to the best operating point as a single-electron source. Opening the $\operatorname{dot}(D=0.8$, right panel) also leads to flat bands as expected but we note that the eigenvalues for the first band (which is the only one that is non-negligible) is only 0.83 .

Going to a closed dot $(D=0.11$, left panel) leads to a curved first band with average 0.5 . This point corresponds to the local maximum of the entropy between

TABLE I. Positions in the $(D, e V / \Delta)$ plane and values of $S_{\mathrm{vN}}$ (in bits) for the entropy minima - crosses on Fig. 5 - in the sinedrive case for $\mathrm{eV} / \Delta \leq 1$ (when about one electron per period is emitted). At each of these operating points, the source emits a single electronic atom of signal per period whose Wigner representation is depicted Fig. 10. The associated hole atom of signal is charge conjugated and shifted by a half-period.

\begin{tabular}{cccc}
\hline \hline & $D$ & $e V / \Delta$ & $S_{\mathrm{VN}}$ \\
\hline 1 & 0.29 & 0.09 & 0.10 \\
2 & 0.38 & 0.24 & 0.06 \\
3 & 0.41 & 0.40 & 0.06 \\
4 & 0.43 & 0.55 & 0.10 \\
5 & 0.42 & 0.70 & 0.18 \\
\hline \hline
\end{tabular}

$D=0$ and $D=D_{\text {opt }}$ along $e V=e V_{\text {opt }}$. At this point, the entropy is equal to 0.85 bit. Starting from the optimal point, decreasing $D$ increases the escape time of the electron and hole excitations. In previous publications $[29,38]$, we had argued that, in a specific regime, the mesoscopic capacitor emits a quantum superposition of nothing and of an elementary electron-hole pair on top of the Fermi sea. Decreasing $D$ would increase the amplitude of the emission of the electron-hole pair from modulus very close to one to modulus zero and this explains the behavior of the entropy with decreasing $D$ at fixed $e V / \Delta$. However, when $D$ is decreased, interperiod coherences (or equivalently band curvature) appear due electron and hole delocalization over more than one half-period. This shows how our analysis unravels what happens more precisely than the previously used simple picture.

\section{Electronic atoms of signal and coherences}

Let us now discuss the electronic atoms of signal as well as their coherence properties at the same three round points. As shown on Fig. 9, for a widely open dot, there is still one type of electronic atom of signal with no interperiod correlations that is emitted per half-period, although it is emitted with a probability less than 1 . When closing the dot, we first encounter an optimal point $(D \sim$ $D_{\text {opt }}$ ) where only one is emitted almost certainly: the mesoscopic capacitor behaves like an almost ideal singleelectron source [see Eq. (5)] and there are no interperiod electronic coherences [see Eq. (6)]. Finally, when closing the dot, the electronic escape time from the dot increases beyond $T / 2$ and, consequently, the elementary electron and hole excitations emitted by the capacitor tends to delocalize over more than one period. Moreover, electron-hole coherences are generated and we encounter a point with a local maximum of electron-hole entanglement $(D \simeq 0.11)$. Analyzing the shape of the Wannier wave packets confirms that closing the dot leads to longer wave packets.

Figure 10 presents the dominant electronic atoms of signal for the local optimal points in the quadrant $0<D<1$

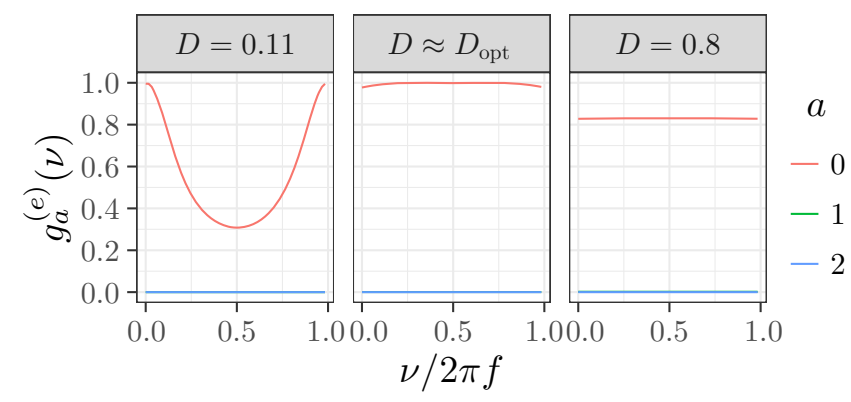

FIG. 8. Floquet-Bloch spectra for the three selected round points in the sine-drive case appearing on Fig. 5. Only the first three bands are represented, all the other ones being even closer to zero. 


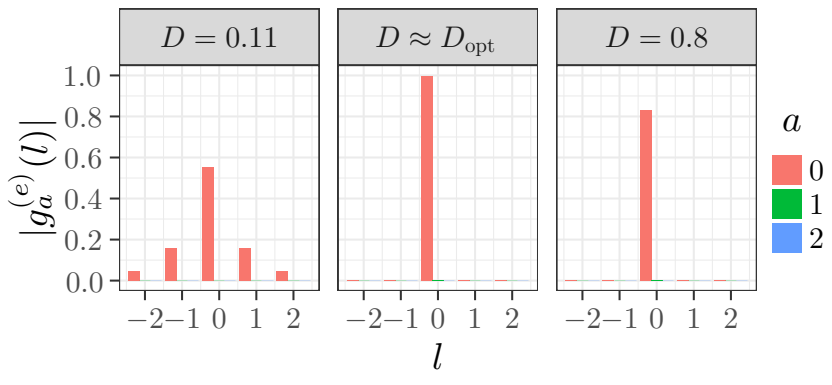

FIG. 9. Modulus of the interperiod coherences $\left|g_{a}^{(e)}(l)\right|$ between the electronic atoms of signal of the $a=0,1$ and 2 Floquet-Bloch bands given by Eq. (19) as a function of $l$ for the three round points on Fig. 5 (sine-drive case).

and $0<e V / \Delta<1$. As we raise the drive amplitude, the Wannier wave functions explore higher energies. For each minimum, there is a corresponding number of negative bumps in the Wigner representation. This suggests that these optimal regimes correspond to a resonance between the rising time of the drive voltage, the period and the energy gap of the cavity.

In conclusion, this analysis demonstrates how the Floquet-Bloch analysis can be used to find optimal operating points of single-electron sources and, more generally, to characterize what is emitted by the source and to optimize wave packets shaping strategies [73]. As such, it can help improving the quantitative modeling of many electron quantum optics experiments such as, for example, electronic decoherence experiments [50]. Because the electronic excitations emitted by the mesoscopic capacitor are quite sensitive to this phenomenon, and also for practical reasons, the Leviton sources built from a suitably driven Ohmic contact [68] are very relevant. We now apply our analysis to this very important source.

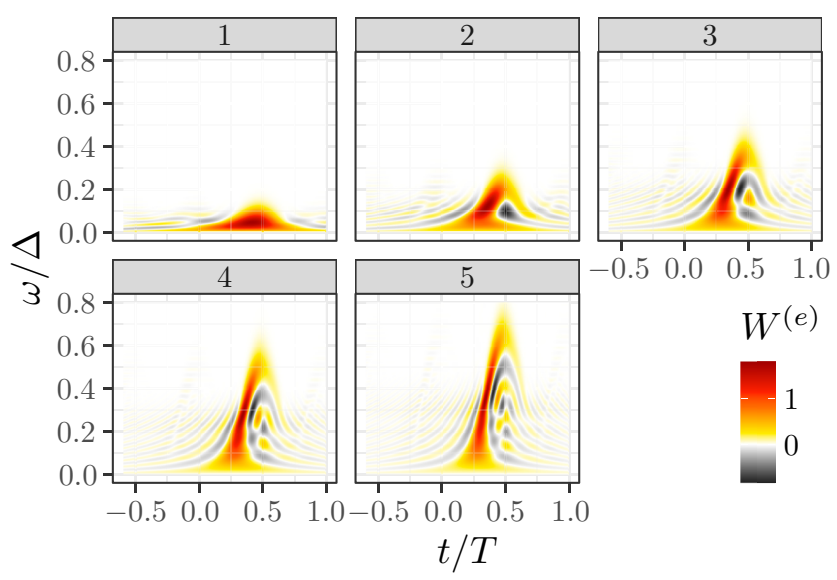

FIG. 10. Dominant electronic atoms of signals emitted by the mesoscopic capacitor for the local minima of $S_{\mathrm{vN}}$ appearing on Fig. 5 (crosses) in the domain $0<D<1$ and $0<\mathrm{eV} / \Delta<1$.

\section{B. Leviton trains}

Let us now consider an Ohmic contact driven by time-dependent voltage, which is a $T$-periodic train of Lorentzian pulses of width $\tau_{0}$, each of them carrying an electric charge $q=-\alpha e$. The resulting time-dependent voltage

$$
V(t)=\frac{\alpha h f}{2 e} \frac{\sinh \left(2 \pi f \tau_{0}\right)}{\sinh ^{2}\left(\pi f \tau_{0}\right)+\sin ^{2}(\pi f t)}
$$

has a dc component $V_{\mathrm{dc}}=\alpha h f / e$ and an ac part $V_{\mathrm{ac}}(t)=$ $V(t)-V_{\mathrm{dc}}[68]$.

To understand the underlying physics, let us remember what happens in the case of a single Lorentzian pulse of duration $\tau_{0}$ and integer charge $\alpha=n>0$ at zero temperature. In this case, the emitted many-body state is a Slater determinant built by adding on the Fermi sea $n$ mutually orthogonal electronic single-electron excitations whose wave functions are given in the frequency domain by [74]

$$
\varphi_{n}(\omega)=\sqrt{4 \pi v_{F} \tau_{0}} H(\omega) L_{n-1}\left(2 \omega \tau_{0}\right) e^{-\omega \tau_{0}},
$$

where $L_{n}$ denotes the $n$th Laguerre polynomial and $H$ is the Heaviside distribution. In the limit where Lorentzian pulses are well separated $\left(f \tau_{0} \ll 1\right)$, we expect the electronic atoms of signal, which we call Levitonoids, to have a strong overlap with these mutually orthogonal wave functions.

For a Leviton train $(\alpha=1)$, one could naively expect each Lorentzian voltage pulse to carry exactly one Leviton excitation. Although the Levitonoid may tend to the isolated Leviton in the limit $\tau_{0} \ll T$, in the case where the Lorentzian pulses start to overlap $\left(f \tau_{0} \gtrsim 1\right)$, the relation between the Levitonoid and the Leviton is nontrivial because of the Pauli principle since single-Leviton wave functions of width $\tau_{0}$ separated by $T \ll \tau_{0}$ are not orthogonal.

To gain a better understanding of the way information is encoded in such a compact electronic train, besides the periodic train of Levitons, we consider in Sec. V B 2 a randomized train of Lorentzian voltage pulses [45], obtained by randomly choosing whether or not each Lorentzian pulse is present in the drive or not. The single-electron coherence associated with this statistical ensemble of voltage drives is still $T$-periodic and our analysis can be applied.

\section{Levitonic atoms of signals}

Figure 11 shows the full Wigner distribution function of the $T$-periodic train of unit-charge Lorentzian pulses for different values of $f \tau_{0}$. Varying this parameter swipes from a dilute train in which each Leviton is well separated from each other, to a compact train in which the pulses are so 


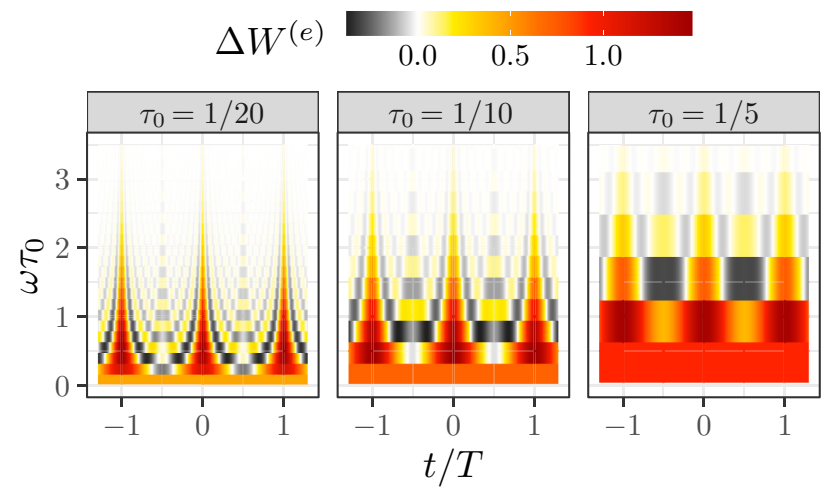

FIG. 11. Wigner distribution function of a Leviton train for different ratio $\tau_{0} / T$ for increasing values of $\tau_{0} / T$. As we raise $\tau_{0} / T$, the duration of each Leviton becomes longer and longer and, compared to the energy scale $\hbar / \tau_{0}, h f$ becomes larger. Once $\tau_{0}$ is greater than $T$, the first band of width $2 \pi f$, which will have no time dependance, is the only one to remain. We are thus left with an almost stationary situation due to the raise of chemical potential by $\delta \mu=h f$.

spread over multiple periods that we see only the variation of the chemical potential due to the dc part.

In the $\alpha=1$ case, Moskalets has obtained explicit expressions for electronic atoms of signal associated with such a Leviton train [47]. Each of them leads to a Lorentzian current pulse of width $\tau_{0}$. This is manifestly not the case for the electronic atoms of signal obtained numerically whose Wigner representations are depicted on Fig. 12. Our numerical algorithm produces wave packets having the smallest spreading in time whereas the analytical expressions obtained by Moskalets possess a Lorentzian current pulse of width $\tau_{0}$. As shown in Appendix $\mathrm{H}$, an analytical expression for the minimally spread wave packets can be obtained:

$$
\varphi_{\mathrm{Lev}}(\omega)=\frac{1}{\sqrt{\mathcal{N}}} H(\omega) e^{-\omega_{\mathrm{int}} \tau_{0}}
$$

where $\mathcal{N}$ is a normalization factor and $\omega_{\text {int }}=2 \pi f$ $\lfloor\omega / 2 \pi f\rfloor$ is the frequency counted in multiples of $2 \pi f$. This minimally spread Levitonoid is the following linear combination of Martin-Landauer's wave packets

$$
|\mathrm{Lev}\rangle=\sqrt{1-e^{-4 \pi f \tau_{0}}} \sum_{n=0}^{+\infty} e^{-2 \pi n f \tau_{0}}\left|\mathrm{ML}_{n, 0}\right\rangle
$$

The details of this derivation can be found in Appendix H. We check that this analytical form and the one found by the algorithm match perfectly.

As can be seen from Fig. 12, the minimally spread Levitonoid $|\mathrm{Lev}\rangle$ tends, in the $f \tau_{0} \rightarrow 0$ limit, to the singleLeviton state $\left|\varphi_{1}\right\rangle$ obtained from Eq. (47): when lowering $f \tau_{0}$, the steps of width $2 \pi f$ in Eq. (48) become smaller,

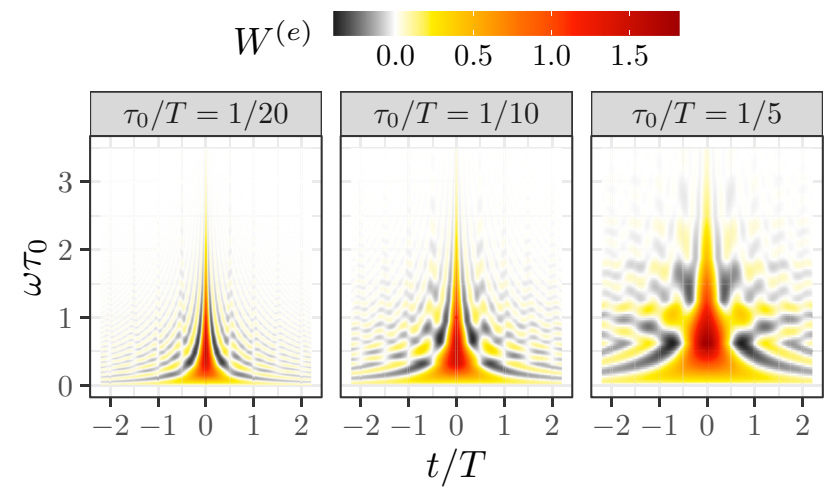

FIG. 12. Electronic atoms of signal of a train of charge $\alpha$ Lorentzian pulses for $\alpha=1$ and different values of $f \tau_{0}$. When $f \tau_{0} \ll 1$, the wave packet we obtain is very similar to a Leviton. When $f \tau_{0} \simeq 1$, we recover a Martin-Landauer wave packet. The atoms of signal found by our algorithm (lower panel) fit perfectly the ones predicted analytically (upper panel).

thereby corresponding to an increasingly closer staircase approximation of the decaying exponential in Eq. (47). A measure of the distance between the minimal Levitonoid $|\mathrm{Lev}\rangle$ and the single-Leviton state $\left|\varphi_{1}\right\rangle$ is given by the overlap between these two single-particle states:

$$
\left|\left\langle\operatorname{Lev} \mid \varphi_{1}\right\rangle\right|^{2}=\frac{\tanh \left(\pi f \tau_{0}\right)}{\pi f \tau_{0}}
$$

which, for $f \tau_{0} \ll 1$, departs quadratically from unity.

In the regime where $f \tau_{0} \simeq 1$, the overlap between the minimal Levitonoid and the single Leviton tends to zero as $1 / \pi f \tau_{0}$. In this regime, it seems natural to compare our Levitonoids to the Martin-Landauer wave packet $\left|\mathrm{ML}_{1,0}\right\rangle$ (compare the right panel of Fig. 12 to Fig. 4). This overlap goes exponentially to one as $f \tau_{0}$ goes to infinity

$$
\left|\left\langle\operatorname{Lev} \mid \varphi_{\mathrm{ML}_{0,0}}\right\rangle\right|^{2}=1-e^{-4 \pi f \tau_{0}}
$$

For the examples discussed above, when $f \tau_{0}=1 / 5$, the overlap is around $92 \%$. At $f \tau_{0}=1$, the overlap is unity up to the sixth significant digit, making the differentiation between a minimally spread Levitonoid and a MartinLandauer impossible in practice.

These behaviors shed light on the difference in terms of typical temporal width between the minimally spread Levitonoids and the wave packets introduced by Moskalets. For Moskalets' wave packets, the typical duration is always $\tau_{0}$. In our case, the typical duration is $\tau_{0}$ when $\tau_{0} \lesssim T$. However, when $\tau_{0} \gtrsim T$, the minimal Levitonoid will have a duration of the order of $T$ and, ultimately, in the $f \tau_{0} \rightarrow \infty$ limit, tends to a Martin-Landauer wave packet. 


\section{The random train}

In order to distinguish between the electronic wave packets used to carry the information and the way they are injected, we elaborate on the recent idea [45] of randomizing the emission process itself. We consider nonperiodic trains of electrons associated with infinite random binary chains $b_{k}(k \in \mathbb{Z})$, which determines whether a Lorentzian pulse centered at $t_{k}=k T$ is added to the driving voltage $\left(b_{k}=1\right.$ with probability $\left.p\right)$ or not $\left(b_{k}=0\right.$ with probability

$$
\mathcal{G}_{R_{p}}^{(e)}\left(t+\frac{\tau}{2} \mid t-\frac{\tau}{2}\right)=\frac{\sin \left\{\pi\left[f t-\theta_{p}(f \tau)\right]\right\} \sin \left\{\pi\left[f t+\theta_{p}(f \tau)\right]\right\}}{\sin \left[\pi f\left(t-i \tau_{0}+\frac{\tau}{2}\right)\right] \sin \left[\pi f\left(t+i \tau_{0}-\frac{\tau}{2}\right)\right]} \mathcal{G}_{F}^{(e)}(\tau)
$$

in which the index $R_{p}$ stands for "randomly emitted with probability $p$ ", $\mathcal{G}_{F}^{(e)}$ denotes the Fermi sea's single-electron coherence and

$$
\theta_{p}(x)=\sqrt{\frac{x^{2}}{4}-\left(f \tau_{0}\right)^{2}-i(1-2 p) f \tau_{0} x} .
$$

Eqs. (53) and (54) are the starting point for applying our Floquet-Bloch analysis for finding the electronic atoms of signals underlying the randomized train of Lorenztian pulses. Remarkably, the analysis can be performed numerically but also analytically, as explained in Appendix I.

The first important result is that the excess singleelectron coherence can be described in terms of minimal Levitonoids, which are the appropriate electronic atoms of signal for the nonrandom $T$-periodic train. This illustrates quantitatively the motivation put forward in Ref. [45]: randomization enables us to separate what is emitted from the way it is emitted. Single-electron coherence is thus described in terms of the same electronic atoms of signals but with a different "quantum coherence score."

More precisely, when the pulses are widely spaced $\left(f \tau_{0} \ll 1\right)$, we can associate a single-electron excitation (the minimal Levitonoid), which is very close to the Leviton wave packet [see Eq. (50)] with each Lorentzian pulse. Lowering $p$ then just lowers the emission probability of the corresponding single-electron excitation (see the left panel of Fig. 13). In this regime, randomization just lowers the intensity of the emission, as would be expected with a classical (incoherent) ensemble of musicians choosing to play, or not to play each note of the score, so that the total intensity of each of them is decreased.

However, for the random Leviton train, lowering the emission probability of each Lorentzian pulses can lead to subtle effects when the Pauli principle starts to enter the game, in the $f \tau_{0} \sim 1$ regime or above. This is the second important result from our detailed analysis: although, the excess single-electron coherence is still described in terms of minimal Levitonoids, lowering $p$ also introduces interperiod coherences depicted on Fig. 13. At fixed $p$, they increase with $f \tau_{0}$ as seen by comparing the three panels of Fig. 13. In this regime, the modification of the "quantum coherence score" induced by lowering $p$ is not naively classical as in the $f \tau_{0} \ll 1$ regime: interperiod coherences are revealed. This can be understood as follows: the limit of a dense $T$-periodic train is recovered for $p \rightarrow 1$ : one Levitonoid is then emitted per period without any interperiod coherences. But then, decreasing $p$ opens some space on the adjacent periods: among all the classical drives building the statistical ensemble underlying $R_{p}$, the weight of those containing pulses separated by more than

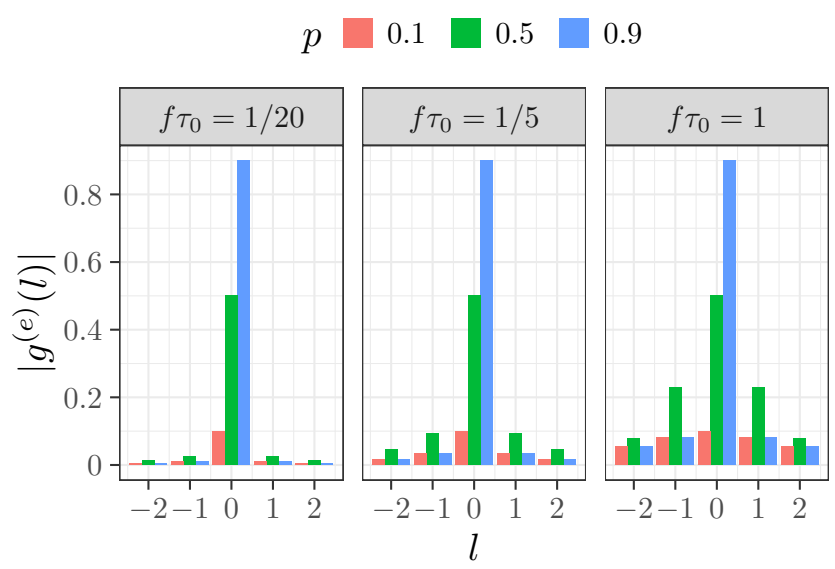

FIG. 13. Coherence between time-shifted Levitonoids for the random train with emission probabilities $p=0.1,0.5,0.9$ and a width $f \tau_{0}=1 / 20,1 / 5,1$. The central peak has value $p$ and we clearly see the increase of interperiod coherences when increasing $f \tau_{0}$ at fixed $p$ and their spreading when decreasing $p$ at fixed $f \tau_{0}$. 


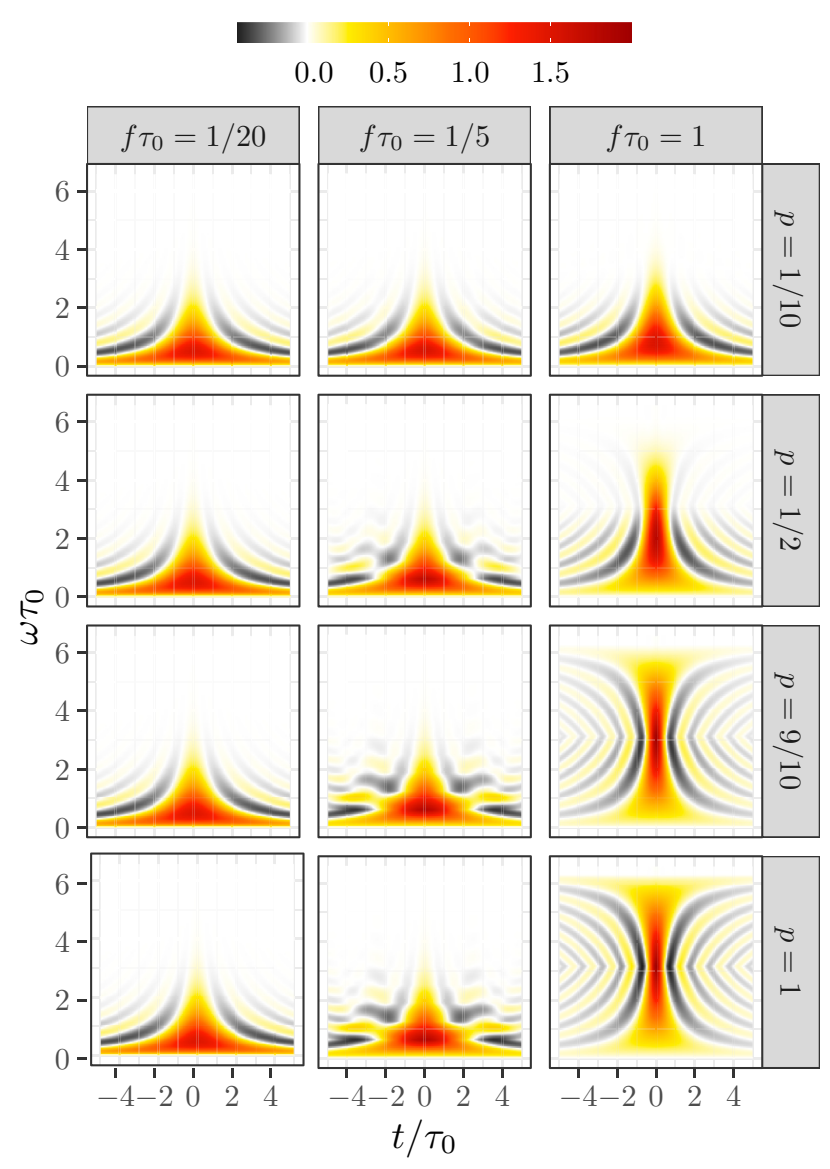

FIG. 14. Wigner representations of $p$-Glattlions for $p=0.1$, $0.5,0.9,1$ and a width $f \tau_{0}=1 / 20,1 / 5$ and 1 . The case $p=1$ corresponds to the minimal Levitonoids introduced in Sec. V B 1. Note that, on this figure, time is counted in units of $\tau_{0}$ so that we clearly see how the $p$-Glattlion interpolates between a Levitonlike Wigner representation of width $\tau_{0}$ for $p=1 / 10$ to a minimal Levitonoid one for $p=9 / 10$.

$T$ increases and this contributes to the increasing weight of interperiod coherences. In the limit $p \rightarrow 0$, we thus expect to recover an excess electronic coherence spreading over $|\tau| \lesssim \tau_{0}$, very similar to the one of an isolated Leviton because, in this limit, the weight of trajectories for which an emitted Lorentzian pulse is separated from the nearest other emitted pulses by more than $\tau_{0}$ goes to unity. This explains the increasing interperiod coherences in the low $p$, high $f \tau_{0}$ regime.

Remarkably, and this is the third result from our in-depth analysis, these interperiod coherences can be recasted in terms of normalized single-electron states, which we call the $p$-Glattlion and which are

$$
\left|\mathrm{Gla}_{p}\right\rangle=\int_{0}^{+\infty} \sqrt{\frac{1-e^{-4 \pi f \tau_{0}}}{p f}} e^{-\omega_{\mathrm{int}} \tau_{0}} \sqrt{g^{(e)}(\omega)}|\omega\rangle d \omega
$$

Using these single-particle states, the excess singleelectron coherence can be written as

$$
\Delta_{0} \mathbf{G}_{R p}^{(e)}=p \sum_{l \in \mathbb{Z}}\left|\mathrm{Gla}_{p, l}\right\rangle\left\langle\mathrm{Gla}_{p, l}\right|
$$

in which $\left|\mathrm{Gla}_{p, l}\right\rangle=\mathbf{T}_{T}^{l}\left|\mathrm{Gla}_{p}\right\rangle$ is the $p$-Glattlion translated by $l T$. This rewriting resums the interperiod coherences in the minimal Levitonoid basis into pure single-electron states. Ultimately, such a rewriting reflects the coherence of the voltage pulse trains used to build the random ensemble $R_{p}$. The price to pay is that these states cannot be viewed as electronic atoms of signals since they are not mutually orthogonal between different periods [see Eq. (I18)]. The Wigner representations of $\mathrm{Gla}_{p}$ for various $\left(p, f \tau_{0}\right)$ are plotted on Fig. 14. These single-particle states interpolate between Leviton wave functions of width $\tau_{0}$ in the limit $f \tau_{0} \ll 1$ or $p \ll 1$ and the minimal Levitonoid obtained for $p=1$.

\section{CONCLUSION AND PERSPECTIVES}

In this work, we introduce a representation of the singleelectron coherence of a periodic electron source in terms of perfectly distinguishable normalized single-particle wave functions associated with each period, which we call "electronic atoms of signals" [29]. This description, which is the counterpart of the Karhunen-Loève decomposition for classical signals [75], enables us to obtain a simple description of the single-particle content emitted by the source in discrete terms. The electronic atoms of signal are the building blocks of the single-electron coherence, which are emitted according to their emission probabilities and quantum coherences. Such a decomposition is very reminiscent of the way music can be described in terms of notes arranged along a specific score: the emission probability being analogous of the strength at which the note is played whereas the coherences are specifically quantum. This type of decomposition, generalized to nonperiodic quantum electrical currents is a convenient way to represent general single-electron quantum signals exactly as a music score represents a generically nonperiodic piece of music. This is the appropriate framework to discuss the encoding and decoding of the quantum information embedded within a quantum electrical current.

Being able to access the single-particle content of a quantum electric current suggests that a very high degree of control may be envisioned in the near future. This is particularly important for the potential applications of electron quantum optics to quantum sensing of electromagnetic fields on a submicrometric space and subnanosecond time scale.

In particular, our study of electron sources also shows that, generically, an electron source emits several electron 
or hole wave functions. The multiplicity of emitted excitations is enhanced by nonzero temperature as shown in the recent experimental study [9]. From a signal-processing point of view, this means that in general, electron quantum optics sources and detectors, respectively, emit and detect many different electron and hole excitations. In this sense, they are quantum counterparts to MIMO multiple-input multiple-output (MIMO) classical microwave devices such as advanced radars and WiFi routers, which make use of many (spatial) modes to improve transmission or detection performances. In the long run, the representation of electronic coherence in terms of electronic atoms of signal will be instrumental for characterizing and improving the performances of quantum-sensing devices based on quantum electric currents, exactly as MIMO is now used in radar technology [76]. It may as well help quantifying and maybe improving quantum-information flow within these devices, as was done in classical signal processing [77].

This decomposition may also bring new insights on physical phenomena such as electron fractionalization $[74,78]$, the effect of temperature on trains of multiparticle states [79] and interaction-induced electronic decoherence $[50,80]$. Since the Floquet-Bloch decomposition provides a zero-order guess for the many-body state from single-particle coherence in the absence of interactions, it is the perfect starting point for a more refined description of the many-body states based, for example, on the adaptation to electron quantum optics of the unitary coupled cluster method now used in variational quantum eigensolvers [81]. Such an ansatz would reproduce deviations from Wick's theorem at higher and higherorder coherences, thereby providing a clear insight of the electronic coherences in terms of the many-body state.

Finally, our quantum analyzer may also offer a way to access to the recently studied electron-hole entanglement [82-84] and, supplemented by other measurements [28], to quantify more precisely the importance of interactioninduced higher-order quantum correlations as well as of thermal fluctuations $[85,86]$.

The general quantum signal-processing method presented here is also directly relevant for electron quantum optics in other systems such as topological insulators [87] and, with some adaptation, in strongly correlated 1D quantum edge channels such as fractional quantum Hall edges [88] where it might shed some light on recently predicted correlation effects within trains of Lorentzian pulses [89]. Finally, it can establish a bridge between electron and microwave quantum optics [90-93], by probing the electronic content of microwave photons injected from a transmission line into a quantum conductor. However, this requires establishing a bridge between the coherence properties of electrons and the quantum optical coherence of the emitted radiation extending the work of Ref. [94].

\section{ACKNOWLEDGMENTS}

We thank E. Bocquillon, P. Borgnat, and P. Flandrin for useful discussions. This work is supported in part by the Agence Nationale de la Recherche (ANR) Grant "1shot reloaded" (ANR-14-CE32-0017), the European Research Council (ERC) Consolidator Grant "EQuO" and by the Joint Research Project "SEQUOIA" (17FUN04) within the European Metrology Programme for Innovation and Research (EMPIR) co-financed by the Participating States and from the European Union's Horizon 2020 research and innovation programme.

\section{APPENDIX A: NORMALIZATIONS}

The single-particle states $|t\rangle$ and $|\omega\rangle$, normalized as

$$
\begin{aligned}
& \left\langle t \mid t^{\prime}\right\rangle=v_{F}^{-1} \delta\left(t-t^{\prime}\right), \\
& \left\langle\omega \mid \omega^{\prime}\right\rangle=\delta\left(\omega-\omega^{\prime}\right)
\end{aligned}
$$

are related by

$$
\begin{gathered}
|t\rangle=\frac{1}{\sqrt{2 \pi v_{F}}} \int d \omega e^{i \omega t}|\omega\rangle, \\
|\omega\rangle=\sqrt{\frac{v_{F}}{2 \pi}} \int d t e^{-i \omega t}|t\rangle .
\end{gathered}
$$

Using the expression of the fermion field operator

$$
\psi(t)=\int_{\mathbb{R}} c(\omega) e^{-i \omega t} \frac{d \omega}{\sqrt{2 \pi v_{F}}}
$$

in terms of fermionic annihilation and creation operators $c(\omega)$ and $c^{\dagger}(\omega)$ obeying the canonical anticommutation relations $\left\{c(\omega), c^{\dagger}\left(\omega^{\prime}\right)\right\}=\delta\left(\omega-\omega^{\prime}\right)$, the $\mathbf{G}^{(e)}$ operator is expressed in the $|\omega\rangle$ base as

$$
\mathbf{G}^{(e)}=\int_{\mathbb{R}^{2}}\left|\omega_{+}\right\rangle\left\langle c^{\dagger}\left(\omega_{-}\right) c\left(\omega_{+}\right)\right\rangle_{\rho}\left\langle\omega_{-}\right| d \omega_{+} d \omega_{-} .
$$

\section{APPENDIX B: FLOQUET-BLOCH THEORY}

\section{Diagonalizing the electron part}

Let us introduce the projectors $\Pi_{ \pm}$on the space of positive (respectively, negative) energy single-particle states. The projections $\mathbf{G}_{\varepsilon, \varepsilon}^{(e)}=\Pi_{\varepsilon} \mathbf{G}^{(e)} \Pi_{\varepsilon}(\varepsilon= \pm 1)$ of the singleelectron coherence operator contain information on electronic excitations for $\varepsilon=+$ and on hole excitations for $\varepsilon=-$. These correspond to the electron and hole quadrants of Fig. 3. In the same way, the off-diagonal parts $\mathbf{G}_{\varepsilon,-\varepsilon}^{(e)}=\Pi_{\varepsilon} \mathbf{G}^{(e)} \Pi_{-\varepsilon}$ couple the electron and hole parts of the single-particle state and encode electron-hole coherences. 
Note that $\mathbf{G}_{++}^{(e)}$ contains all the electronic excitations, even the thermal ones that are present at nonzero temperature when the source is switched off. Keeping these is essential for having positive operators to diagonalize. Denoting by $\Delta_{0} \mathbf{G}_{(e)}=\mathbf{G}^{(e)}-\mathbf{G}_{F}^{(e)}=\mathbf{G}^{(e)}-\Pi_{-}$, we have

$$
\mathbf{G}^{(e)}=\Pi_{-}+\mathbf{G}_{++}^{(e)}+\Delta_{0} \mathbf{G}_{--}^{(e)}+\mathbf{G}_{+-}^{(e)}+\mathbf{G}_{-+}^{(e)},
$$

where $\mathbf{G}_{--}^{(e)}=\Pi_{-}+\Delta_{0} \mathbf{G}_{--}^{(e)}$ and $\mathbf{G}_{++}^{(e)}=\Delta_{0} \mathbf{G}_{++}^{(e)}$ (same for +- and -+ ).

The first step consists in diagonalizing the electron part of the excess single-electron coherence $\mathbf{G}_{++}^{(e)}$. Since $\mathbf{G}^{(e)}$ is Hermitian as well as $\Pi_{+}, \mathbf{G}_{++}^{(e)}$ is also Hermitian. Since $\left[\Pi_{+}, \mathbf{T}_{T}\right]=0, \mathbf{G}_{++}^{(e)}$ commutes with $\mathbf{T}_{T}$ and we also know that it is a positive operator bounded by 1 . Therefore, $\mathbf{G}_{++}^{(e)}$ and $\mathbf{T}_{T}$ can be diagonalized simultaneously. Exactly as in solid-state theory, the diagonalization is performed on each of the eigenspaces of $\mathbf{T}_{T}$, which consist in quasiperiodic single-particle states associated with a quasienergy $0 \leq$ $v<2 \pi f(f=1 / T)$ and corresponding to the eigenvalue $e^{-i \nu T}$ of $\mathbf{T}_{T}$. The spectrum for $\mathbf{G}_{++}^{(e)}$ has a band structure with eigenvalues $g^{(e)}(v) \in[0,1]$ for $0 \leq v<2 \pi f$. We can, therefore, find an orthogonal basis of eigenvectors $\left|\psi_{a, v}^{(e)}\right\rangle \in \mathcal{H}_{+}$such that

$$
\begin{gathered}
\mathbf{T}_{T}\left|\psi_{a, \nu}^{(e)}\right\rangle=e^{-i v T}\left|\psi_{a, \nu}^{(e)}\right\rangle, \\
\mathbf{G}_{++}^{(e)}\left|\psi_{a, \nu}^{(e)}\right\rangle=g_{a}^{(e)}(\nu)\left|\psi_{a, \nu}^{(e)}\right\rangle .
\end{gathered}
$$

These eigenvectors are called the electronic Floquet-Bloch vectors and we can choose them to satisfy the normalization conditions

$$
\left\langle\psi_{a, v}^{(e)} \mid \psi_{a^{\prime}, v^{\prime}}^{(e)}\right\rangle=2 \pi \delta_{a, a^{\prime}} \delta\left(v-v^{\prime}\right),
$$

which is the same as the $\sqrt{2 \pi}|\omega\rangle$ states. The explicit form of the eigenvalue equations used in the numerical computation is discussed in Appendix B 4. It relies on the decomposition of each Floquet-Bloch state as a sum of plane waves whose energies differ by a multiple of $h f$ :

$$
\left|\psi_{a, \nu}^{(e)}\right\rangle=\sum_{n=0}^{+\infty} u_{a, v}^{(n)}|v+2 \pi n f\rangle .
$$

The main difference with the usual Bloch theory in solidstate physics comes from the fact that, here, the sum is restricted to $n \in \mathbb{N}$ because we are considering electronic excitations.

\section{Hole excitations and electron-hole coherences}

Having discussed the electronic part of the singleelectron coherence, let us discuss the hole part as well as the electron-hole part. We can introduce a hole operator $\mathbf{G}^{(h)}$ defined by replacing $\mathcal{G}_{\rho, x}^{(e)}\left(t, t^{\prime}\right)$ in Eq. (11) by

$$
\mathcal{G}_{\rho, x}^{(h)}\left(t, t^{\prime}\right)=\operatorname{tr}\left[\psi^{\dagger}(x, t) \rho \psi\left(x, t^{\prime}\right)\right]
$$

This operator satisfies the same mathematical properties as $\mathbf{G}^{(e)}$. This can be easily shown by using the anticommutation relations of fermionic operators to relate electron and hole coherence operators:

$$
\mathbf{G}^{(h)}=\mathbf{1}-\mathbf{C G}^{(e)} \mathbf{C}^{\dagger},
$$

where $\mathbf{C}$ is the antiunitary involution that transforms electrons in holes and vice versa, defined in the time basis by complex conjugation: $\langle t \mid \mathbf{C} \psi\rangle=\langle t \mid \psi\rangle^{*}$. We then have $\mathbf{G}^{(h)}=\boldsymbol{\Pi}_{-}-\mathbf{C} \Delta_{0} \mathbf{G}^{(e)} \mathbf{C}^{\dagger}$ and, therefore, holes can be dealt with along the same lines as electronic excitations.

However, rather than focusing on the restriction of $\mathbf{G}^{(h)}$ to the positive frequencies quadrant, it turns out to be more convenient to focus on $\Delta_{0} \mathbf{G}_{--}^{(e)}$ defined as the restriction to the negative frequencies' quadrant of $\Delta_{0} \mathbf{G}^{(e)}=\mathbf{G}^{(e)}-$ $\boldsymbol{\Pi}_{-}$. Taking differences with respect to the $\mu=0$ Fermi sea ensures that all excitations, including thermal ones, are taken into account. Then $\Delta_{0} \mathbf{G}_{--}^{(e)}$ contains eigenfunctions of holes at negative frequencies, with eigenvalues that are the opposite of hole occupation numbers.

Exactly as $\mathbf{G}_{++}^{(e)}, \mathbf{G}_{--}^{(e)}$ can be diagonalized simultaneously with $\mathbf{T}_{T}$. We thus introduce an eigenbasis of hole single-particle states $\left|\psi_{b, v}^{(h)}\right\rangle \in \mathcal{H}_{-}$such that

$$
\Delta_{0} \mathbf{G}_{--}^{(e)}=-\sum_{b} \int_{0}^{2 \pi f} g_{b}^{(h)}(\nu)\left|\psi_{b, v}^{(h)}\right\rangle\left\langle\psi_{b, v}^{(h)}\right| \frac{d \nu}{2 \pi} .
$$

Using the completion relation

$$
\Pi_{-}=\sum_{b} \int_{0}^{2 \pi f}\left|\psi_{b, v}^{(h)}\right\rangle\left\langle\psi_{b, v}^{(h)}\right| \frac{d \nu}{2 \pi},
$$

the hole part $\mathbf{G}_{--}^{(e)}$ is then diagonal in the $\left|\psi_{b, v}^{(h)}\right\rangle$ basis with respective eigenvalues $1-g_{b}^{(h)}(v)$ :

$$
\mathbf{G}_{--}^{(e)}=\sum_{b} \int_{0}^{2 \pi f}\left[1-g_{b}^{(h)}(\nu)\right]\left|\psi_{b, \nu}^{(h)}\right\rangle\left\langle\psi_{b, \nu}^{(h)}\right| \frac{d \nu}{2 \pi} .
$$

This convention for the hole Floquet-Bloch spectrum ensures that $\mathbf{G}_{++}^{(h)}$ is diagonalized by the eigenvectors $\mathbf{C}\left|\psi_{b, v}^{(h)}\right\rangle$ with respective eigenvalue $g_{b}^{(h)}(v)$. Let us notice 
that, for $0 \leq v<2 \pi f$, the hole eigenstate decomposition into plane wave takes the form

$$
\left|\psi_{b, \nu}^{(h)}\right\rangle=\sum_{n=1}^{+\infty} v_{b, v}^{(n)}|v-2 \pi f n\rangle
$$

in order to include only negative-energy plane waves. The final step for deriving Eq. (15) is to introduce the electron-hole coherences in the basis of electronic-hole Floquet-Bloch eigenstates:

$$
\begin{aligned}
& \left\langle\psi_{a, \nu}^{(e)}\left|\mathbf{G}^{(e)}\right| \psi_{b, v^{\prime}}^{(h)}\right\rangle=2 \pi \delta\left(v-v^{\prime}\right) g_{a b}^{(e h)}(v), \\
& \left\langle\psi_{b, v}^{(h)}\left|\mathbf{G}^{(e)}\right| \psi_{a, v^{\prime}}^{(e)}\right\rangle=2 \pi \delta\left(v-v^{\prime}\right) g_{b a}^{(h e)}(v) .
\end{aligned}
$$

\section{Floquet-Bloch eigenvalues as occupation numbers}

The normalization condition, Eq. (14), for the eigenstates $\left|\psi_{a, v}^{(e)}\right\rangle$ is the same as the one of plane waves except for the fact that, in the present case, $v$ is a quasimomentum living in $\mathbb{R} / 2 \pi f \mathbb{Z}$. The destruction operator associated with such an excitation is thus defined by direct analogy with the operator $c(\omega)$ :

$$
\psi\left[\psi_{a, \nu}^{(e)}\right]=\frac{v_{F}}{\sqrt{2 \pi}} \int_{-\infty}^{+\infty} \psi_{a, \nu}^{(e)}(t)^{*} \psi(t) d t,
$$

where the normalization factor ensures the canonical anticommutation relation

$$
\left\{\psi\left[\psi_{a, v}\right], \psi^{\dagger}\left[\psi_{a^{\prime}, v^{\prime}}\right]\right\}=\delta_{a, a^{\prime}} \delta\left(v-v^{\prime}\right) .
$$

It then follows that

$$
\left\langle\psi^{\dagger}\left[\psi_{a^{\prime}, v^{\prime}}^{(e)}\right] \psi\left[\psi_{a, v}^{(e)}\right]\right\rangle=\delta_{a, a^{\prime}} \delta\left(v-v^{\prime}\right) g_{a}^{(e)}(v) .
$$

This equation is analogous to the expression of the singleelectron coherence of a stationary state in the basis of fixed-energy single-particle states $|\omega\rangle$ in terms of the electron distribution function $f_{e}(\omega)$ :

$$
\left\langle c^{\dagger}\left(\omega^{\prime}\right) c(\omega)\right\rangle=\delta\left(\omega-\omega^{\prime}\right) f_{e}(\omega) .
$$

The eigenvalues $g_{a}^{(e)}(v)$ can thus be interpreted as the occupation numbers of the single-particle states $\left|\psi_{a, v}^{(e)}\right\rangle$. We can, therefore, interpret the spectrum of $\mathbf{G}_{++}^{(e)}$ as bands of occupation numbers for the Floquet-Bloch states $\left|\psi_{a, v}^{(e)}\right\rangle$ as a function of their quasienergy $v \in \mathbb{R} / 2 \pi f \mathbb{Z}$. In the same way, the bands $v \mapsto g^{(h)}(v)$ can be interpreted as giving the occupation numbers for the hole excitations $\mathbf{C}\left|\psi_{b, v}^{(h)}\right\rangle$, which are quantum superpositions of single-particle states with energies $h f-\hbar v$ shifted by positive multiples of $h f$.

Of course, this raises the question of the band structure that can occur in this type of problem. In order to get a hint on this question, we must have a closer look at the underlying eigenvalue problems.

\section{Eigenvalue equations}

The diagonalization problem that leads to the spectrum $\left[g_{a}(v)\right]_{a, v}$ and to the Floquet-Bloch eigenfunctions is best expressed in the frequency domain $[38,40]$. Exactly as in Bloch's theory, we introduce $T$-periodic dimensionless functions $u_{a, v}$ such that $\psi_{a, v}(t)=e^{-i v t} v_{F}^{-1 / 2} u_{a, v}(t)$. Choosing a representative of the quasienergy $v \in[0,2 \pi f$ [, we decompose $u_{a, v}(t)$ in Fourier series

$$
u_{a, \nu}(t)=\sum_{n=0}^{+\infty} u_{a, \nu}^{(n)} e^{-2 i \pi n f t}
$$

where the sum goes from $n=0$ to $n=+\infty$ since we are looking for purely electronic wave functions so each $v+$ $2 \pi n f$ is positive. The eigenvalue equation $\mathbf{G}_{++}^{(e)}\left|\psi_{a, \nu}\right\rangle=$ $g_{a}^{(e)}(v)\left|\psi_{a, v}\right\rangle$ can then be rewritten in terms of the singleelectron coherence projected onto the electron quadrant. With our choice of a representative $v \in[0,2 \pi f$ [ for the quasienergy, the eigenvector equation for $g_{a}^{(e)}(v)$ is

$$
\sum_{p \in \mathbb{N}} W_{++, n-p}^{(e)}[v+\pi f(n+p)] u_{a, v}^{(p)}=g_{a}^{(e)}(v) u_{a, v}^{(n)},
$$

where, because of $T$ periodicity, we decompose the Wigner distribution function $W_{++}^{(e)}(t, \omega)$ associated with $\mathbf{G}_{++}^{(e)}$ as a Fourier series:

$$
W_{++}^{(e)}(t, \omega)=\sum_{n \in \mathbb{Z}} e^{-2 \pi i n f t} W_{++, n}^{(e)}(\omega) .
$$

Note that, because we are considering the projection onto the electronic quadrant, $W_{++, n}^{(e)}(\omega)=0$ for $\omega-\pi|n| f<0$ and equal to $W_{n}^{(e)}(\omega)$ the $n$th harmonic of the full Wigner function, for $\omega-\pi|n| f \geq 0$. Equation (B17) is solved numerically to determine the spectrum of the singleelectron coherence restricted to the electronic quadrant. We can also see it as the diagonalization of the matrix $M(v)$, defined for each $v \in[0,2 \pi f$ [ as

$$
M_{n p}(\nu)=W_{++, n-p}^{(e)}[v+\pi f(n+p)]
$$

for $(n, p) \in \mathbb{N}^{2}$ This matrix is thus derived from the energy representation of the first-order coherence as graphically pictured on Fig. 15.

The eigenvalues for the hole Floquet-Bloch matrix are obtained in the same way starting from Eq. (B10) and following the same step very precisely. This leads to the eigenvalue equation $\left(0 \leq v<2 \pi f\right.$ and $\left.n \in \mathbb{N}^{*}\right)$ :

$$
\sum_{p=1}^{+\infty} W_{--, p-n}^{(e)}[v-\pi f(n+p)] v_{b, v}^{(p)}=\left[1-g_{b}^{(h)}(v)\right] v_{b, v}^{(n)}
$$

in which 


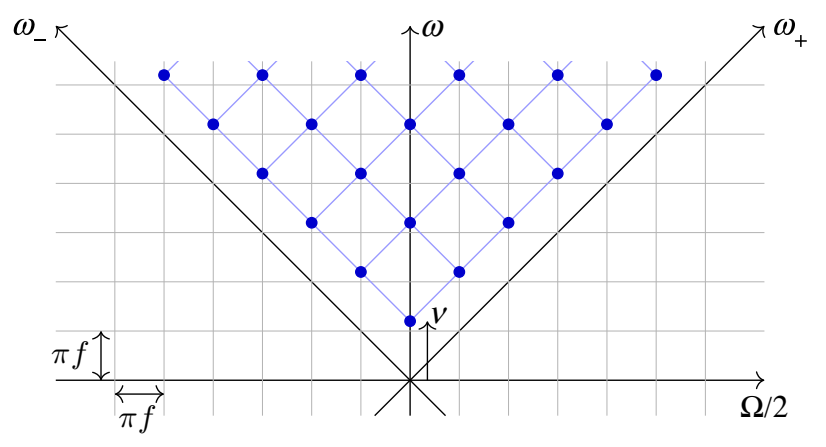

FIG. 15. Graphical representation of the matrix $M(v)$. The first-order coherence in energy representation takes values for $\delta v$ being an integer multiple of $\pi f$. The matrix we extract at a given frequency $v$ is the one given by the value of the blue dots, that are spaced by integer multiples of $2 \pi f$ in both $\omega_{ \pm}$directions. Shifting the frequency $v$ by $\delta v$ corresponds to vertically translating all blue dots by $\delta v$.

$$
W_{--}^{(e)}(t, \omega)=\sum_{n \in \mathbb{Z}} e^{-2 \pi i n f t} W_{--, n}^{(e)}(\omega)
$$

is the Wigner distribution function associated with $\mathbf{G}_{--}^{(e)}$ and, therefore, $W_{--, n}^{(e)}(\omega)=0$ for $\omega+\pi|n| f>0$ and is equal to $W_{n}^{(e)}(\omega)$ as soon as $\omega+\pi|n| f \leq 0$.

\section{The case of a voltage drive at zero temperature}

A specific feature of the case of a time-dependent classical drive is that, at zero temperature, the energy coherence is piecewise constant, the width of each step being $2 \pi f$. If we consider a purely ac drive, the discontinuities do not appear when we extract the matrix $M(v)$ for $v \in[0,2 \pi f[$ (see Fig. 16, left). As such, the eigenvalues will be independent on the quasienergy, and the eigenvectors of different quasienergy can be deduced by a frequency translation. It implies notably that it is possible to find a set of FloquetWannier functions that are piecewise constant in energy, with discontinuities happening every $2 \pi f$. Consequently, the electronic atoms of signal are linear combinations of Martin-Landauer wave packets, a point already noticed in
Ref. [3], and there are no interperiod coherences due to band flatness.

If we add a dc part to the voltage, then it will shift the whole energy coherence by $\mu / \hbar$ (see Fig. 16 , right). In this case, there are two possibilities:

(a) If $\mu / h f$ is an integer, we are back to the ac case, since the discontinuity will not happen for $v \in$ $[0,2 \pi f[$.

(b) If $\mu / h f$ is not an integer, then the matrix $M(v)$ will be piecewise constant, with a step at $v=$ $\mu / \hbar[2 \pi f]$. Similarly, the eigenvectors for $v \in$ $\left[0, \omega_{s}[\right.$ can be deduced by translating the eigenvectors at $v=0$ in energy. The eigenvectors for $v \in$ $\left[\omega_{s}, 2 \pi f[\right.$ can be deduced by translating the eigenvectors at $\omega_{s}$. In this case, we can find a set of Floquet-Wannier functions that are piecewise constant in energy, with steps happening at $2 \pi n f$ and $2 \pi n f+\omega_{s}$.

If we consider a small, nonzero temperature, such that $k_{B} T_{\text {el }} \ll h f$, the steps will be smoothed out over a scale $k_{B} T_{\mathrm{el}} / \hbar$. We can thus expect that the property mentioned above remains true, except at the neighborhood of discontinuities.

This example demonstrates that, contrary to the case of bands in solid-state physics, the Floquet-Bloch bands we are considering here may exhibit discontinuities that, indeed, may play a crucial role. For example, this is the case when applying a dc-voltage bias corresponding to a noninteger multiple of $-e f \mathrm{dc}$ current to an ac voltage drive or, more generally, to a purely ac source. An example is a periodic train of Lorentzian voltage pulses carrying a noninteger charge in units of $-e$.

\section{APPENDIX C: FLOQUET-WANNIER FUNCTION AMBIGUITIES}

In this appendix, we discuss the ambiguities in the determination of electronic atoms of signals and propose a

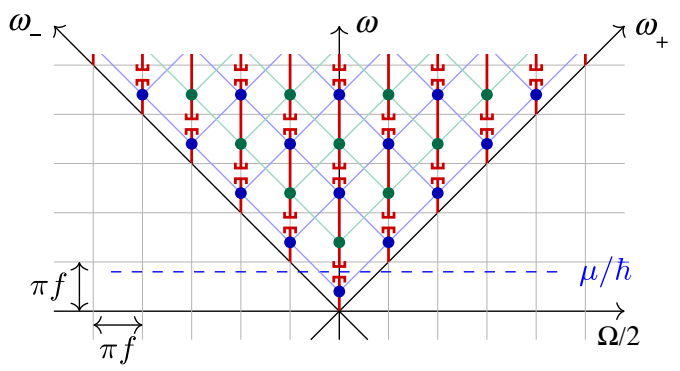

FIG. 16. Matrices for a voltage drive at zero temperature. On the left, the case of an ac voltage drive. In this case, the coherence is constant for all $v \in[0,2 \pi f$ [. The eigenvalue problem does not depend any more on the quasienergy. On the right, we consider that there is a dc part on top of the ac voltage. In this case, the matrix $M(v)$ will be piecewise constant, with a step at $\nu=\mu / \hbar(\bmod 2 \pi f)$. 
minimal-spreading principle for selecting a specific choice of electronic atoms of signals.

\section{Origin of the ambiguities}

Ambiguities in the choice of Floquet-Wannier wave functions can always be traced back to degenerate common eigenspaces for $\mathbf{G}_{++}^{(e)}$ and $\mathbf{T}_{T}$. Let us introduce a unitary transformation $\mathbf{U}$ that keeps $\mathbf{G}_{++}^{(e)}$ eigenspaces stable: $\left[\mathbf{U}, \mathbf{G}_{++}^{(e)}\right]=0$, then using the $\mathbf{U}\left|\psi_{a, \nu}\right\rangle$ states in Eq. (16), we obtain a new orthonormal family of Wannier functions, which we denote by $\left|\varphi_{a, l}^{[U]}\right\rangle$. Equation (17) becomes

$$
\mathbf{T}_{T}\left|\varphi_{a, l}^{[\mathbf{U}]}\right\rangle=\left|\varphi_{a, l+1}^{\left[\mathbf{T}_{T} \mathbf{U} \mathbf{T}_{T}^{\dagger}\right]}\right\rangle .
$$

In order to satisfy the time-translation property of Wannier wave functions, Eq. (17), we require that $\mathbf{U}$ preserves each eigenspace of $\mathbf{T}_{T}$ and we then discuss what happens depending on the structure of the common eigenspaces of $\mathbf{T}_{T}$ and $\mathbf{G}_{++}^{(e)}$.

Preserving the eigenspaces of $\mathbf{T}_{T}$ immediately implies that $\mathbf{U}$ preserves quasienergy eigenspaces. Assuming that it leaves each of them invariant, this means that it reduces to a unitary transformation operating on the space generated by all the Floquet-Bloch states at a given quasienergy. Let us now analyze what happens depending on the eigenspaces of $\mathbf{G}_{++}^{(e)}$ at fixed quasienergy.

In the case where the Floquet-Bloch bands are nondegenerate and do not cross, each common eigenspace is one dimensional and the only possibility for redefining the Floquet-Bloch eigenstates is to introduce quasienergydependent phases:

$$
\left|\psi_{a, v}\right\rangle \mapsto e^{i \theta_{a}(v)}\left|\psi_{a, \nu}\right\rangle .
$$

Such quasienergy-dependent phases $\theta(v)$ fall into different topological sectors, which are labeled by the winding number

$$
n_{W}=\frac{1}{2 \pi} \int_{0}^{2 \pi f} \frac{d \theta(\nu)}{d \nu} d \nu .
$$

For example $\theta_{n}(v)=n T \nu$ has winding number $n \in \mathbb{Z}$ and Eq. (16) implies that

$$
\left|\varphi_{a, l}^{\left[e^{i \theta_{n}}\right]}\right\rangle=\left|\varphi_{a, l+n}\right\rangle .
$$

Consequently, a topologically nontrivial phase has the same effect as combining a translation by an integer number of periods with a topologically trivial energydependent phase.

In the case of $n$ degenerate Floquet-Bloch bands over the whole quasienergy interval, the above phases are replaced by a quasienergy-dependent unitary transformation $U(v) \in$ $\mathrm{U}(n)$ for $0 \leq v<2 \pi f$ so that, considering $A_{\alpha}$ the set of $n$ band indexes, the new Wannier functions are defined by

$$
\left|\varphi_{a, l}^{[U]}\right\rangle=\frac{1}{\sqrt{f}} \int_{0}^{2 \pi f} \sum_{b \in A_{\alpha}} U_{a, b}(\nu)\left|\psi_{b, \nu}\right\rangle \frac{d \nu}{2 \pi} .
$$

Such transformations are directly relevant when a source emits $n$ single-electron excitations on top of the Fermi sea. In this case $g_{a}(v)=1$ for several values of $a$. The topological sectors of such quasienergy-dependent unitaries are classified by the topological sectors of the overall phase since all groups $\mathrm{SU}(n \geq 2)$ are simply connected. Let us now discuss in more detail the properties of the FloquetBloch band structure to see which situation is more likely to be encountered.

A first observation is that bands may have discontinuities. From our observations for classical voltage drives and for the mesoscopic capacitor driven by a sinusoidal or square voltage, it seems that such discontinuities appear when temperature is nonzero, for purely ac sources.

Finally, since physical states are defined up to a phase, we have an extra possibility for defining electronic atoms of signals in the case of flat bands. For example, one could replace Eq. (17) by its projective version, that is introducing a phase in front of $\left|\varphi_{a, l+1}\right\rangle$. Combining this with Eq. (C1) leads to

$$
U_{\Omega}\left|\psi_{a, v}\right\rangle=\left|\psi_{a, v+\Omega}\right\rangle,
$$

where the addition is considered modulo $2 \pi f(\Omega \in \mathbb{R} /$ $2 \pi f \mathbb{Z}$ ). Substituting this into Eq. (16) leads to

$$
\left|\varphi_{a, l}^{\left[U_{\Omega}\right]}\right\rangle=e^{i \Omega T}\left|\varphi_{a, l+1}\right\rangle .
$$

The time-translation property, Eq. (17), is satisfied up to a phase.

\section{Minimal-spreading principle}

Let us now discuss the general method used to determine suitable electronic atoms of signals. Exactly as in solid-state physics, a natural idea is to look for maximally localized Wannier functions [53]. Let us consider $\varphi_{a}$ such a wave function, the spreading $\left\langle(\Delta t)^{2}\right\rangle_{\varphi_{a}}$ is defined as

$$
\begin{aligned}
\left\langle(\Delta t)^{2}\right\rangle_{\varphi_{a}}= & v_{F} \int_{\mathbb{R}} t^{2}\left|\varphi_{a, 0}(t)\right|^{2} d t \\
& -\left[v_{F} \int_{\mathbb{R}} t\left|\varphi_{a, 0}(t)\right|^{2} d t\right]^{2} .
\end{aligned}
$$

Let us consider directly the case of $n$ degenerated bands $g_{a}(v)=p_{\alpha}(v)$ for all $0 \leq v<2 \pi f$ and $a \in A_{\alpha}$. 
We then have a quasienergy-dependent unitary transformation ambiguity described by Eq. (C5). Maximally localized Wannier wave functions are now found by minimizing the quadratic functional

$$
\mathcal{S}[U]=\sum_{a \in A_{\alpha}}\left\langle(\Delta t)^{2}\right\rangle_{\left|\varphi_{a}^{[U]}\right\rangle}
$$

over $U(v) \in \mathcal{U}(n)$ for $0 \leq v<2 \pi f$. Note that the righthand side of Eq. (C8) may be divergent due to the large time behavior of $\left|\varphi_{a, 0}(t)\right|^{2}$ as, for example, in the case of a Leviton train. In such a case, we should, therefore, regularize it by subtracting the same quantity for a reference choice of the unitary operator such as $U(v)=\mathbf{1}$.

Numerically, the implementation of the minimization process is straightforward in the case of a nondegenerate band. Since there is a natural cutoff for the length of the wave packet, in this case it is easy to compute the functional, Eq. (C8), from an arbitrary phase, Eq. (C2). More importantly, it is also easy to compute the gradient, giving access to all efficient gradient-based minimization algorithms. In our case, we rely on the GNU Scientific Library (GSL) implementation of the Fletcher-Reeves algorithm [95]. It consists in a succession of line minimizations. We begin at a given point (which can either be a random phase or a null phase), and the first direction of minimization is given by the gradient. Then, at each iteration, a new direction is chosen, depending on the previous search direction, the gradient of current iteration and the norm of the gradient of previous iteration. The iteration ends when the gradient is orthogonal to the line of search.

For the degenerate case, Eq. (C5), there are several difficulties. First, we need to parametrize the unitary matrices $U(v)$. For this, we introduce $\Theta(v)$, Hermitian matrices such that

$$
U(v)=\exp [i \Theta(v)]
$$

The main difficulty here is that, since $\mathrm{U}(n \leq 2)$ is a noncommutative group, it becomes hard to compute the gradients of the functional $\mathcal{S}[U]$. However, it is still easy to compute them if we consider a starting point at $U=\mathbb{1}$. In the following, we denote $\left|\psi_{A, v}\right\rangle$ the vector containing every wave function $\left|\psi_{a, v}\right\rangle$ with $a \in A, A$ being the degenerate set of bands we want to minimize on. The matrix $U(v)$ acts on this vector space, mixing wave functions. At each iteration $n>1$ of the algorithm, we now replace the wave functions $\left|\psi_{A, v}^{(n-1)}\right\rangle$ by the wave functions

$$
\left|\psi_{A, v}^{(n)}\right\rangle=e^{i x_{n} H_{n}(v)}\left|\psi_{A, v}^{(n-1)}\right\rangle,
$$

$H_{n}$ being the search direction and $x_{n}$ the real parameter that minimize this search direction. This allows us to always start the line-minimization process from $U=\mathbb{1}$.
To determine the minimum, we check whether our search direction is orthogonal to the local gradient computed by shifting $e^{i x_{n} H_{n}(v)}$ to identity. What makes everything work is that all quantities needed to compute the new direction of minimization are either invariant on the point of the $\mathrm{U}(n)$ group we consider them (norm of the previous gradient), computed locally (the new gradient) or trivially transported (previous search direction, which is parallel to the transport). After $N$ iterations, we end up with

$$
\left|\psi_{A, v}^{(N)}\right\rangle=e^{i \Theta_{N}(v)} \cdots e^{i \Theta_{1}(v)}\left|\psi_{A, v}^{(0)}\right\rangle
$$

emphasizing the noncommutative character of the group we are minimizing on.

\section{APPENDIX D: HBT AND HOM CURRENT NOISE}

\section{Explicit expressions}

The outgoing current correlation $S_{11}^{(\text {out })}\left(t, t^{\prime}\right)=\left\langle i_{1 \text { out }}(t)\right.$ $\left.i_{1 \text { out }}\left(t^{\prime}\right)\right\rangle-\left\langle i_{\text {1out }}(t)\right\rangle\left\langle i_{\text {1out }}\left(t^{\prime}\right)\right\rangle$ after a quantum point contact whose scattering matrix is

$$
\left(\begin{array}{ll}
\sqrt{\mathcal{R}} & i \sqrt{\mathcal{T}} \\
i \sqrt{\mathcal{T}} & \sqrt{\mathcal{R}}
\end{array}\right)
$$

has been computed in Ref. [38] in terms of the incoming current correlators and single-electron coherences:

$$
S_{11}^{(\text {out })}\left(t, t^{\prime}\right)=\mathcal{R}^{2} S_{11}^{(\mathrm{in})}\left(t, t^{\prime}\right)+\mathcal{T}^{2} S_{22}^{(\mathrm{in})}\left(t, t^{\prime}\right)+\mathcal{R} \mathcal{T} \mathcal{Q}\left(t, t^{\prime}\right)
$$

in which

$$
\mathcal{Q}\left(t, t^{\prime}\right)=e^{2} v_{F}^{2}\left\{\mathcal{G}_{1}^{(e)}\left(t^{\prime}, t\right) \mathcal{G}_{2}^{(h)}\left(t^{\prime}, t\right)+[1 \leftrightarrow 2]\right\}
$$

encodes two-particle interference effects between the two incoming channels. The excess current noise is, therefore, given by

$$
\begin{gathered}
\Delta S_{11}^{\text {(out) }}\left(t, t^{\prime}\right)=\mathcal{R}^{2} \Delta S_{11}^{(\mathrm{in})}\left(t, t^{\prime}\right)+\mathcal{T}^{2} \Delta S_{22}^{(\mathrm{in})}\left(t, t^{\prime}\right) \\
+\mathcal{R} \mathcal{T} \Delta \mathcal{Q}\left(t, t^{\prime}\right)
\end{gathered}
$$

Hanbury Brown and Twiss experiments correspond to one of the sources being switched on and the other one being switched off. From now on, let us assume that both sources $S_{1}$ and $S_{2}$ are identical and synchronized. Under this hypothesis, $\Delta S_{11}^{(\mathrm{in})}=\Delta S_{22}^{(\mathrm{in})}=\Delta S_{S}$ is the excess noise generated by the source.

At zero temperature, the HBT excess zero frequency current noise can be expressed using the Floquet-Bloch 
spectrum as $\mathcal{R} \mathcal{T} \Delta \mathcal{Q}_{\mathrm{HBT}}$ where

$$
\Delta \mathcal{Q}_{\mathrm{HBT}}=e^{2} \int_{0}^{2 \pi f}\left[\sum_{a} g_{a}^{(e)}(v)+\sum_{b} g_{b}^{(h)}(v)\right] \frac{d v}{2 \pi}
$$

in which $\Delta \mathcal{Q}_{\text {HBT }}$ [see Eq. (D3b)] arises from the partitioning of electron and hole excitations at the QPC not contained in the partitioning of the incoming current noises [rhs of Eq. (D3a) at zero frequency]. This leads to Eq. (29a).

When both sources are switched on, a Hong-Ou-Mandel experiment is performed. Using Eq. (D3), the corresponding excess noise is the sum of the excess noise of the two possible HBT experiments

$$
\Delta S_{11}^{(\text {out })}=\Delta S_{1}^{(\mathrm{HBT})}+\Delta S_{2}^{(\mathrm{HBT})}+\mathcal{R} \mathcal{T} \Delta \mathcal{Q}_{\mathrm{HOM}}
$$

and of a two-excitations interference contribution involving the two sources $S_{1}$ and $S_{2}$, which can be expressed as

$$
\begin{aligned}
\Delta \mathcal{Q}_{\mathrm{HOM}}= & -2 e^{2} \int_{0}^{2 \pi f}\left[\sum_{a} g_{a}^{(e)}(v)^{2}+\sum_{b} g_{b}^{(h)}(v)^{2}\right] \frac{d \nu}{2 \pi} \\
& -4 e^{2} \sum_{a, b} \int_{0}^{2 \pi f}\left|g_{a b}^{(e h)}(v)\right|^{2} \frac{d \nu}{2 \pi}
\end{aligned}
$$

using the Floquet-Bloch analysis of the single-electron coherence emitted by the source $S_{1}$, identical to $S_{2}$ here. Adding twice the rhs of Eq. (D4) (one for each source) to the rhs of Eq. (D6) leads to the total contribution $\left(\mathcal{R}^{2}+\right.$ $\left.\mathcal{T}^{2}\right) \Delta S_{S}$ to the zero-frequency current noise that comes on top of the partitioning of the sources intrinsic excess current noise $\Delta S_{S}$. In the end, as shown on Fig. 17, the final expression (28c) for the excess zero frequency current noise at the HOM dip is the sum of the excess current noise of the sources transmitted by the two sources (which is always positive), to which is added the total two-excitation interference contribution given by Eq. (29b).

\section{The HOM dip}

Let us now use this to discuss the depth of the HOM dip defined as the difference between the HOM excess noise given by Eq. (D5) and the sum of the two HBT noises. Counting the dip's depth positively, its expression is

$$
\left[\Delta S_{\text {dip }}\right]=-\mathcal{R} \mathcal{T} \Delta Q_{\mathrm{HOM}}
$$

where $\Delta Q_{\mathrm{HOM}}$ is given by Eq. (D6).

It is interesting to rewrite these expressions in terms of physically more appealing quantities. For this, let us

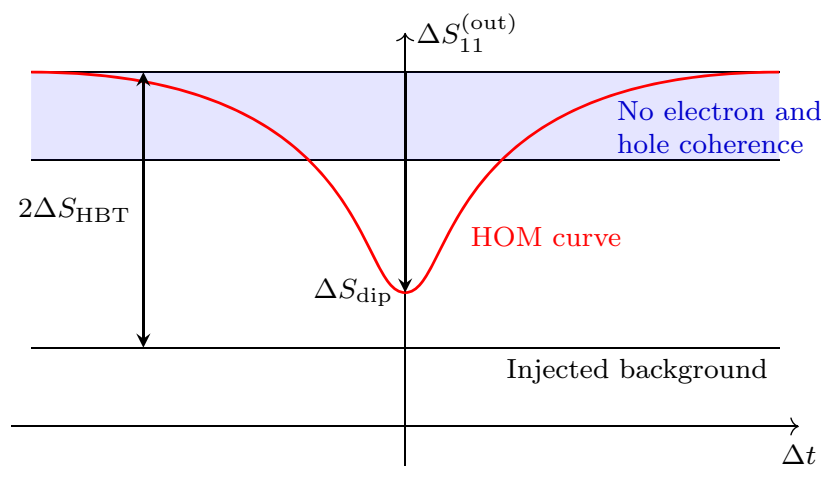

FIG. 17. Depth of the HOM dip at zero temperature: in a HOM experiment, the excess low-frequency current noise is above the background noise $\left(\mathcal{R}^{2}+\mathcal{T}^{2}\right) \Delta S_{S}$ injected by the sources. When the sources, which emit localized excitations, are sufficiently desynchronized, the current noise is expected to reach $\Delta S_{\mathrm{HBT}}=e^{2} f \mathcal{R} \mathcal{T}\left(\bar{N}_{e}+\bar{N}_{h}\right)$ exceeding this background noise by $2 \Delta S_{\mathrm{HBT}}$. At fixed Floquet-Bloch spectra $\left[g_{a}^{(e)}(v), g_{b}^{(h)}(v)\right]$, the depth of the HOM dip has a lower bound, which translates into an upper bound for the excess noise equal to $2 e^{2} f \mathcal{R} \mathcal{T}\left[\left(\Delta N_{e}\right)_{W}^{2}+\right.$ $\left(\Delta N_{h}\right)_{W}^{2}$ ] above the injected background noise (light blue zone). This bound is reached for vanishing electron-hole coherences.

introduce an infrared regularization to compute traces of $T$-periodic operators. Let

$$
\mathbf{X}=\sum_{a} \int_{0}^{2 \pi f} X_{a}(v) \frac{d v}{2 \pi}\left|\psi_{a}^{(e)}(v)\right\rangle\left\langle\psi_{a}^{(e)}(v)\right|
$$

be a diagonal operator in the basis of electronic FloquetBloch eigenstates. The trace of this operator, which is defined and acts on $\mathcal{H}_{+}$is divergent. Nevertheless, we can regularize it. Inverting Eq. (16), its expression in the basis formed by the electronic atoms of signals is

$$
\mathbf{X}=\sum_{a} \sum_{\left(l_{+}, l_{l} \in \mathbb{Z}^{2}\right.} X_{a}\left(l_{+}-l_{-}\right)\left|\varphi_{a, l_{+}}\right\rangle\left\langle\varphi_{a, l_{-}}\right| .
$$

in which $X_{a}(l)$ is related to $X_{a}(v)$ by Eq. (19). Taking the trace over a subspace generated by the electronic atoms of signal over a range of $N$ periods gives

$$
\operatorname{Tr}_{N}(\mathbf{X})=N \sum_{a} \int_{0}^{2 \pi f} X_{a}(v) \frac{d v}{2 \pi f} .
$$

This leads to the definition of the per-period regularized trace as $\bar{X}=\operatorname{Tr}_{N}(\mathbf{X}) / N$. With this definition, the average number of electronic and hole excitations emitted per period are given by

$$
\bar{N}_{e}=\sum_{a} \int_{0}^{2 \pi f} g_{a}^{(e)}(v) \frac{d \nu}{2 \pi f},
$$




$$
\bar{N}_{h}=\sum_{b} \int_{0}^{2 \pi f} g_{b}^{(h)}(v) \frac{d \nu}{2 \pi f}
$$

The quadratic terms in the eigenvalues or in the electronhole coherences appearing in Eq. (D6) correspond to what would be obtained, assuming Wick's theorem be valid. Therefore, we denote these quantities with a " $W$ " index. Using Wick's theorem with $\mathbf{G}^{(e)}$ as single-electron coherence, the second moments of the numbers of excitations emitted per periods would be given by

$$
\begin{aligned}
& \left(\Delta N_{e}\right)_{W}^{2}=\sum_{a} \int_{0}^{2 \pi f} g_{a}^{(e)}(v)\left[1-g_{a}^{(e)}(v)\right] \frac{d v}{2 \pi f} \\
& \left(\Delta N_{h}\right)_{W}^{2}=\sum_{b} \int_{0}^{2 \pi f} g_{b}^{(h)}(v)\left[1-g_{b}^{(h)}(v)\right] \frac{d v}{2 \pi f} \\
& \operatorname{Cov}\left(N_{e}, N_{h}\right)_{W}=\sum_{a, b} \int_{0}^{2 \pi f}\left|g_{a b}^{(e h)}(v)\right|^{2} \frac{d v}{2 \pi f}
\end{aligned}
$$

Let us stress that, in the presence of interactions, these are not the actual moments of the numbers of electronic and hole excitations. The actual values differ from these Wick values by a contribution arising from the difference between the intrinsic excess second-order coherence introduced in Ref. [28] and its expected value from Wick's theorem.

The absolute upper bound of the depth of the HOM dip is given by the HBT contribution $\left[\Delta S_{\text {dip }}^{(\max )}\right]=$ $2 e^{2} f \mathcal{R} \mathcal{T} \bar{N}_{\text {tot }}$, where $\bar{N}_{\text {tot }}=\bar{N}_{e}+\bar{N}_{h}$ represents to average total number of excitations emitted per period. Using the above notations, the difference between the maximum dip and the actual dip is then equal to

$$
\begin{aligned}
& {\left[\Delta S_{\mathrm{dip}}^{(\max )}\right]-\left[\Delta S_{\mathrm{dip}}\right]} \\
& =2 e^{2} f \mathcal{R} \mathcal{T}\left[\left(\Delta N_{e}\right)_{W}^{2}+\left(\Delta N_{e}\right)_{W}^{2}-2 \operatorname{Cov}\left(N_{e}, N_{h}\right)_{W}\right] .
\end{aligned}
$$

The rhs is, therefore, directly proportional to the fluctuation $(\Delta Q)_{W}^{2}$ of the excess charge emitted per period by the source. In units of $-e$, the excess charge operator is given by

$$
\widehat{Q}=\int_{\mathbb{R}}: c^{\dagger}(\omega) c(\omega): d \omega,
$$

where the fermionic normal ordering is relative to the reference Fermi sea at chemical potential $\mu=0$. Consequently, the ratio of the dip to its absolute upper bound is given by

$$
\frac{\left[\Delta S_{\mathrm{dip}}\right]}{\left[\Delta S_{\mathrm{dip}}^{(\max )}\right]}=1-\frac{(\Delta Q)_{W}^{2}}{\bar{N}_{\text {tot }}}
$$

If the many-body state does satisfy Wick's theorem, which is the case whenever interactions can be neglected, then having a maximally deep HOM dip corresponds to the actual vanishing charge fluctuations.

\section{APPENDIX E: MANY-BODY STATE AND FLOQUET SCATTERING THEORY}

\section{The floquet-bloch many-body state}

In this appendix, we discuss the connection between our approach and the $T$-periodic single-electron scattering theory that transforms the Fermi sea at chemical potential $\mu=0$ into a pure many-body state. This corresponds to writing down the explicit form of the many-body operator $\mathcal{S}$ whose action corresponds to single-particle scattering

$$
\psi_{\text {out }}(t)=\mathcal{S} \psi_{\text {in }}(t) \mathcal{S}^{\dagger}=\int S\left(t, t^{\prime}\right) \psi_{\text {in }}\left(t^{\prime}\right) d t
$$

where $S\left(t, t^{\prime}\right)$ denotes the $T$-periodic single-particle scattering matrix in the time-domain representation. Without lack of generality, we consider here the case of an ac Floquet source, that is a single-particle scattering operator $\mathcal{S}$ leading to a vanishing average dc current.

Finding an expression for $\mathcal{S}$ is important for two reasons: first it gives insights on the action of Floquet sources on the incoming equilibrium state at the many-body level, then is enables us to connect the form of the many-body operator to the Floquet-Bloch spectrum and eigenstates and electron-hole coherences between them.

\section{a. Two modes}

Let us first discuss the simple two-mode case where only one electron mode $\varphi_{e}$ and one hole mode $\varphi_{h}$ are considered. At zero temperature, the incoming hole mode $\varphi_{h}$ is filled and contains exactly one electron whereas the incoming electron mode $\varphi_{e}$ is empty. The Floquet source will scatter the mode $\varphi_{h}$ into a linear combination of $\varphi_{e}$ and $\varphi_{h}$.

We show that the operator $\mathcal{S}$ can be written as

$$
\begin{gathered}
\mathcal{S}=\mathcal{S}_{d} \mathcal{S}_{p}, \\
\mathcal{S}_{d}=\exp \left(\lambda \psi^{\dagger}\left[\varphi_{e}\right] \psi\left[\varphi_{h}\right]-\lambda^{*} \psi^{\dagger}\left[\varphi_{h}\right] \psi\left[\varphi_{e}\right]\right), \\
\mathcal{S}_{p}=\exp \left(i\left\{\theta_{e}\left(\psi^{\dagger} \psi\right)\left[\varphi_{e}\right]+\theta_{h}\left(\psi^{\dagger} \psi\right)\left[\varphi_{h}\right]\right\}\right) .
\end{gathered}
$$

In this decomposition, $\mathcal{S}_{d}$ is a displacement-like operator, with complex parameter $\lambda$. It corresponds to the scattering 
processes of between the electron and hole modes. $\mathcal{S}_{p}$ is a phase-shifting operator, that independently the phases of the incoming electron and hole modes. Since we can reabsorb the phase of $\lambda$ by changing the relative phase between the wave functions $\varphi_{e}$ and $\varphi_{h}$, we consider $\lambda \in \mathbb{R}^{+}$.

Let us first focus on the displacement-like operator. The exponential can be expanded using the following identity:

$$
\begin{aligned}
& -\left(\psi^{\dagger}\left[\varphi_{e}\right] \psi\left[\varphi_{h}\right]-\psi^{\dagger}\left[\varphi_{h}\right] \psi\left[\varphi_{e}\right]\right)^{2} \\
& =n_{e}\left(1-n_{h}\right)+n_{h}\left(1-n_{e}\right)=\Pi_{\text {odd }}
\end{aligned}
$$

where $n_{e / h}=\left(\psi^{\dagger} \psi\right)\left[\varphi_{e / h}\right]$ denotes the number operator for the corresponding $\varphi_{e / h}$ mode and $\Pi_{\text {odd }}$ is the projector on the one-particle sector. If we also introduce the orthogonal projector $\Pi_{\text {even }}$ that projects on the zero or two-particle sector, a simple expression for the many-body scattering operator follows:

$$
\begin{aligned}
\mathcal{S}_{d}= & \Pi_{\text {even }}+\Pi_{\text {odd }}\{\cos \lambda \\
& \left.+\sin \lambda\left(\psi^{\dagger}\left[\varphi_{e}\right] \psi\left[\varphi_{h}\right]-\psi^{\dagger}\left[\varphi_{h}\right] \psi\left[\varphi_{e}\right]\right)\right\} .
\end{aligned}
$$

Applying the operator $\mathcal{S}$ to the creation operators leads to

$$
\begin{aligned}
\mathcal{S} \psi^{\dagger}\left[\varphi_{e}\right] \mathcal{S} & =\cos \lambda e^{i \theta_{e}} \psi^{\dagger}\left[\varphi_{e}\right]-\sin \lambda e^{i \theta_{h}} \psi^{\dagger}\left[\varphi_{h}\right], \\
\mathcal{S} \psi^{\dagger}\left[\varphi_{h}\right] \mathcal{S} & =\cos \lambda e^{i \theta_{h}} \psi^{\dagger}\left[\varphi_{h}\right]+\sin \lambda e^{i \theta_{e}} \psi^{\dagger}\left[\varphi_{e}\right] .
\end{aligned}
$$

The outgoing creation operators are thus linear combinations of the incoming creation operators. The corresponding linear operator involved is indeed unitary and any unitary operator can be brought in that form by tuning the phase between the wave functions $\varphi_{e}$ and $\varphi_{h}$.

\section{b. The many-mode case}

To understand the full many-body case, we first recast our Floquet-Bloch analysis in terms of the scattering operator. For this, we split the scattering operator into two parts. The first one rearranges electrons and holes inde-

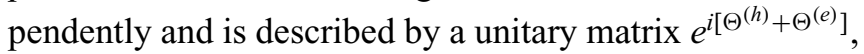
where $\Theta^{(h)}$ and $\Theta^{(e)}$ are Hermitian matrices acting on the hole and electron subspaces, respectively. These operators generalize the phases $\theta_{h}$ and $\theta_{e}$ of the previously discussed two-mode example. Their action on the Fermi sea is, as we see, to add a global phase to the many-body state. The second part involves a two-mode scattering process, in which each pair of modes is scattered according such that

$$
\begin{aligned}
& \mathcal{S} \psi^{\dagger}\left[\varphi_{e}\right] \mathcal{S}^{\dagger}=u \psi^{\dagger}\left[\varphi_{e}\right]+v \psi^{\dagger}\left[\varphi_{h}\right], \\
& \mathcal{S} \psi^{\dagger}\left[\varphi_{h}\right] \mathcal{S}^{\dagger}=u \psi^{\dagger}\left[\varphi_{h}\right]-v \psi^{\dagger}\left[\varphi_{e}\right],
\end{aligned}
$$

with $u, v \in \mathbb{R}_{+}$. The mathematical details for such a decomposition of general unitary operators can be found in Appendix E 2.

The final result of this procedure is an expression of the full many-body scattering operator as a product of uncoupled elementary two-mode operators, with a prefactor that scatters electron and hole subspaces independently:

$$
\begin{aligned}
\mathcal{S}= & \exp \left(\sum_{a \in \mathbb{N}} \int_{0}^{2 \pi f} \lambda_{a}(\nu)\left\{\psi^{\dagger}\left[\psi_{a, \nu}^{(e)}\right] \psi\left[\psi_{a, \nu}^{(h)}\right]-\psi^{\dagger}\left[\psi_{a, \nu}^{(h)}\right] \psi\left[\psi_{a, \nu}^{(e)}\right]\right) \frac{d \nu}{2 \pi}\right) \\
& \times \exp \left(i \sum_{a, b \in \mathbb{N}} \int_{0}^{2 \pi f}\left\{\Theta_{a b}^{(e)}(\nu) \psi^{\dagger}\left[\psi_{a, \nu}^{(e)}\right] \psi\left[\psi_{b, \nu}^{(e)}\right]+\Theta_{a b}^{(h)}(\nu) \psi^{\dagger}\left[\psi_{a, \nu}^{(h)}\right] \psi\left[\psi_{b, \nu}^{(h)}\right]\right\} \frac{d \nu}{2 \pi}\right) .
\end{aligned}
$$

Furthermore, when the bands are flat, we can reorganize a combination of Floquet-Bloch modes as a combination of electronic atoms of signals directly at the many-body level. Of course the choice of Floquet-Wannier functions in the electron quadrant will constrain the choice of FloquetWannier functions in the hole quadrant. Namely, we have

$$
\int_{0}^{2 \pi f} \psi^{\dagger}\left[\psi_{a, \nu}^{(e)}\right] \psi\left[\psi_{a, \nu}^{(h)}\right] \frac{d \nu}{2 \pi}=\sum_{l \in \mathbb{Z}} \psi^{\dagger}\left[\varphi_{a, l}^{(e)}\right] \psi\left[\varphi_{a, l}^{(h)}\right]
$$

\section{Splitting unitary matrices}

In this appendix, we introduce a decomposition for unitary matrices useful when we partition equally the Hilbert space in two. In what remains, we consider a matrix $S \in$ $\mathrm{U}(2 n)$, acting on a Hilbert space $\mathcal{H}=\mathcal{H}_{+} \oplus \mathcal{H}_{-}$, where $\operatorname{dim} \mathcal{H}_{+}=\operatorname{dim} \mathcal{H}_{-}=n$. The goal here is to show that there exists an orthogonal change of basis $P=P_{-} P_{+}$that acts independently on $\mathcal{H}_{+}$and $\mathcal{H}_{-}$in which we can write

$$
P S P^{\dagger}=\left(\begin{array}{c|c}
u & v \\
\hline v & -u
\end{array}\right) e^{i\left(\Theta_{-}+\Theta_{+}\right)},
$$


where $u, v \in \mathcal{M}_{n}$ are positive real diagonal matrices, $\Theta_{ \pm}$ are Hermitian matrices of size $n$. The first block column corresponds to the Hilbert space $\mathcal{H}_{-}$and the second one corresponds to $\mathcal{H}_{+}$.

Generically, one can write the $S$ matrix as

$$
S=\left(\begin{array}{l|l}
S_{--} & S_{-+} \\
\hline S_{+-} & S_{++}
\end{array}\right) .
$$

For the sake of simplicity, we consider that each submatrix is invertible. Other cases would correspond to either fully scattered modes or fully reflected modes, which can be separated from the start without many problems.

We first introduce the polar decomposition of $S_{--}=$ $H_{--} e^{i \theta_{-}}$, where $\theta_{-}$is Hermitian and $H_{--}$is a positive semidefinite Hermitian matrix. This allows us to rewrite $S$ as

$$
S=\left(\begin{array}{c|c}
H_{--} & S_{-+} \\
\hline S_{+-} e^{-i \theta_{-}} & S_{++}
\end{array}\right) e^{i \theta_{-} .}
$$

$H_{--}$being positive semidefinite, we can write it as $H_{--}=$ $P_{-}^{\dagger} u P_{-}$, where $u$ is a diagonal, real-valued, positive matrix. This leads us to

$$
P_{-} S P_{-}^{\dagger}=\left(\begin{array}{c|c}
u & S_{-+}^{\prime} \\
\hline S_{+-}^{\prime} & S_{++}
\end{array}\right) e^{i \Theta},
$$

where $\Theta_{-}=P_{-} \theta_{-} P_{-}^{\dagger}, S_{+-}^{\prime}=P_{-} S_{+-} e^{-i \theta_{-}}$and $S_{-+}^{\prime}=$ $S_{-+} P_{-}^{\dagger}$. The first matrix of the rhs must be unitary. Since $u$ is diagonal, it imposes that each column of $S_{+-}^{\prime}$ is orthogonal to each other. As such, we can rewrite this matrix as a product of a unitary matrix and a diagonal positive real matrix, $S_{+-}^{\prime}=P_{+}^{\dagger} v$. Noting $P=P_{-} P_{+}$, we show

$$
P S P^{\dagger}=\left(\begin{array}{c|c}
u & S_{-+}^{\prime \prime} \\
\hline v & S_{++}^{\prime}
\end{array}\right) e^{i \Theta-},
$$

where $S_{-+}^{\prime \prime}=S_{-+}^{\prime} P_{+}^{\dagger}, S_{++}^{\prime}=P_{+} S_{++} P_{+}^{\dagger}$.

We can now use the Hermitian properties of unitary matrices to build explicitly the constraints between $u, v$, $S_{++}^{\prime}$, and $S_{-+}^{\prime \prime}$. The orthogonality constraint gives us

$$
S_{++}^{\prime}=-(u / v) S_{-+}^{\prime \prime},
$$

where $u / v$ is the diagonal matrix formed by $u v^{-1}$. Conversely, the normalization conditions give us

$$
S_{++}^{\prime}\left[\mathbb{1}_{n}+(u / v)^{2}\right] S_{++}^{\prime}=\mathbb{1}
$$

Since $u^{2}+v^{2}=\mathbb{1}$, this shows that $v^{-1} S_{++}^{\prime}=e^{i \Theta_{+}}$, where $\Theta_{+}$is a Hermitian matrix. Putting everything together, we have

$$
P S P^{\dagger}=\left(\begin{array}{c|c}
u & v \\
\hline v & -u
\end{array}\right) e^{i\left(\Theta_{-}+\Theta_{+}\right)} .
$$

This is the property we want to show.

\section{Many-body state at nonzero temperature}

At nonzero temperature, all terms of Eq. (E9) will play a role. The contribution Eq. (E9b), arising from the separate rearrangement of electron and hole modes will have a nontrivial contribution to the total state. This contribution may scatter electrons deep into the Fermi sea compared to the thermal scale into the thermal fluctuations. It will as well scatter holes from the thermal fluctuations deeper into the Fermi sea. It is also possible to rearrange wave functions inside the thermal band. Similar processes appear in the electron subspace. Notably, this term will explicitly couple different bands. The contribution Eq. (E9a) will also act differently, since sectors of even parities are expected if one of the Floquet-Bloch waves possesses thermal fluctuations at this point. We expect that the atoms of signal, as well as their respective coherences to be modified by this term.

Remarkably, the description in terms of Floquet-Bloch waves at zero temperature allows us to give a manybody description up to the two Hermitian operators $\Theta^{(e)}$ and $\Theta^{(h)}$. This is interesting since it gives a way to see which processes will occur when "heating" an ideal single-electron source. Our approach may thus lead to new insights on the effect of nonzero temperatures on electronic correlations studied in Refs. $[85,86]$.

\section{APPENDIX F: THE PURITY INDICATOR}

Wick's theorem is valid whenever the many-body state $\rho$ of the electron fluid is Gaussian

$$
\rho=\frac{e^{-\psi^{\dagger} \cdot \mathbf{K} \cdot \psi}}{Z_{K}},
$$

where $Z_{\mathbf{K}}=\operatorname{Tr}\left(e^{-\psi^{\dagger} \cdot \mathbf{K} \cdot \psi}\right)$ is the corresponding partition function. The $\mathbf{K}$ operator is related to the single-electron coherence through

$$
\mathbf{G}^{(e)}=\left(\mathbf{1}+e^{\mathbf{K}}\right)^{-1} .
$$

The many-body "unregularized" purity indicator $\mathbb{P}_{\rho}^{(\text {un })}=$ $\operatorname{Tr}\left(\rho^{2}\right)$ can then be formally rewritten as a quotient on infinite-dimensional determinants over the single-particle space of states $\mathcal{H}_{1 \mathrm{p}}$. Using Eq. (F2), it can then be conveniently expressed in terms of the total single-electron coherence:

$$
\begin{gathered}
\mathbb{P}_{\rho}^{(\mathrm{un})}=\frac{\operatorname{Det}\left(\mathbf{1}+e^{2 \mathbf{K}}\right)}{\operatorname{Det}\left(\mathbf{1}+e^{\mathbf{K}}\right)^{2}} \\
=\operatorname{Det}\left(\mathbf{1}+2\left\{\left[\mathbf{G}^{(e)}\right]^{2}-\mathbf{G}^{(e)}\right\}\right) .
\end{gathered}
$$

We can now use the decomposition of the total single-electron coherence $\mathbf{G}^{(e)}=\Pi_{h}+\Delta_{0} \mathbf{G}^{(e)}$, where $\Pi_{h}$ 
denotes the projection onto the space of hole excitations and

$$
\Delta_{0} \mathbf{G}^{(e)}=\left(\begin{array}{cc}
\mathbf{g}_{e} & \mathbf{g}_{e h} \\
\mathbf{g}_{h e} & -\mathbf{g}_{h}
\end{array}\right)
$$

is the excess single-electron coherence with respect to the Fermi sea to obtain an expression for the purity indicator only in terms of data that can be reconstructed by the single-electron tomography protocol. These are $\mathbf{g}_{e}, \mathbf{g}_{h}$ and the off-diagonal parts $\mathbf{g}_{e h}$ and $\mathbf{g}_{h e}$. This finally leads to

$$
\mathbb{P}_{\rho}^{(\mathrm{un})}=\left|\begin{array}{cc}
\mathbf{1}-2\left[\mathbf{g}_{e}\left(\mathbf{1}-\mathbf{g}_{e}\right)-\mathbf{g}_{e h} \mathbf{g}_{h e}\right] & 2\left[\mathbf{g}_{e h} \mathbf{g}_{h}-\mathbf{g}_{e} \mathbf{g}_{e h}\right] \\
2\left[\mathbf{g}_{h} \mathbf{g}_{h e}-\mathbf{g}_{h e} \mathbf{g}_{e}\right] & \mathbf{1}-2\left[\mathbf{g}_{h}\left(\mathbf{1}-\mathbf{g}_{h}\right)-\mathbf{g}_{h e} \mathbf{g}_{e h}\right]
\end{array}\right| .
$$

The final step involves using the fact that, for a $T$ periodic sources, all these operators are block diagonal with respect to the decomposition of the single-particle state into subspaces indexed by the quasienergy $v \in$ $[0,2 \pi f[$. Let us consider a block-diagonal one-particle operator $\mathbf{M}$ block diagonal with respect to the quasienergy $v$. Its determinant can be approximated by discretizing the first Floquet-Brillouin zone $[0,2 \pi f[$ :

$$
\ln \left[\prod_{n=0}^{N-1} \operatorname{Det}\left(\mathbf{M}_{\frac{2 \pi n f}{N}}\right)\right] \simeq N \int_{0}^{2 \pi f} \operatorname{Tr}\left[\ln \left(\mathbf{M}_{\nu}\right)\right] \frac{d \nu}{2 \pi f}
$$

in which $\mathbf{M}_{v}$ precisely denotes the restriction of $\mathbf{M}$ to the subspace of single-particle state with quasienergy $v$. Such a discretization corresponds to juxtaposing $N$ periods of duration $T$ and considering states with periodic boundary conditions on this interval, thereby introducing a formal IR regularization.

This finally gives us the following compact expression for the many-body purity indicator:

$$
\begin{aligned}
\mathbb{P}_{\rho}= & \exp \left(\int_{0}^{2 \pi f} \ln \{1-2 A(v)[1-A(v)]\right. \\
& \left.-2 B(v)[1-B(v)]\} \frac{d v}{2 \pi f}\right)
\end{aligned}
$$

in which

$$
\begin{aligned}
& A(v)=g^{(e e)}(v)\left[1-g^{(h h)}(v)\right]-\left|g^{(e h)}(v)\right|^{2} \\
& B(v)=g^{(h h)}(v)\left[1-g^{(e e)}(v)\right]-\left|g^{(e h)}(v)\right|^{2}
\end{aligned}
$$

are computed in terms of the eigenvalues $g^{(e e)}(v)$ and $g^{(h h)}(v)$ obtained from our diagonalization algorithm (see Sec. III) and of the corresponding electron-hole coherences $g^{(e h)}(v)$. Discussion of the conditions for unit purity can be found in Ref. [9]: it corresponds to a pure many-body state of the form

$$
|\Psi\rangle=\prod_{0 \leq v<2 \pi f}\left\{u(v)+v(v) \psi^{\dagger}\left[\varphi_{\nu}^{(e)}\right] \psi\left[\varphi_{\nu}^{(h)}\right]\right\}|F\rangle,
$$

where $|u(v)|^{2}+|v(v)|^{2}=1$ for all $0 \leq v<2 \pi f$.

\section{APPENDIX G: MESOSCOPIC CAPACITOR: CASE OF A SQUARE DRIVE}

In the case of a square drive used to demonstrate singleelectron emission by the mesoscopic capacitor [1], the $T$-periodic voltage drive is defined by $V_{g}(t)=-V / 2$ for $-T / 2 \leq t<0$ and $V_{g}(t)=V / 2$ for $0<t<T / 2$.

\section{Electron-hole entanglement}

Figure 18 presents a density plot of the entropy defined by Eq. (43) as a function of $D$ and $e V / \Delta$ at fixed $\Delta / h f=20$. There are shallow zones with minima in each square $e V / \Delta \in] n, n+1](n \in \mathbb{N})$ and $0<D \leq 1$. In the single-electron sector, a global minimum can be found at $e V_{\text {opt }} / \Delta \approx 0.37$ and $D_{\text {opt }} \approx 0.47$ and the corresponding entropy is 0.20 bit. As we see, this is the regime where the mesoscopic capacitor behaves almost ideally, emitting exactly one electron and one hole excitation per period.

The minimal entropy reached here is higher than in the sine-drive case, thereby meaning that we are a bit further away from the ideal single-electron and hole-source regime than for the sine-drive case. However, one should keep in mind that the square and the sine drive emit quite different electronic wave functions as discussed below. This means that these different drives are indeed relevant for different applications.

There is also a minimum in the second square where $1<e V / \Delta \leq 2$ but the zone is further from zero. In this zone, three electrons are emitted during the first half period and three holes during the other one, due to the fact that at zero voltage, there is a level at the Fermi energy. It is not surprising that in this zone the deviation from the ideal regime is greater than in the previous case, since we expect a generation of more electron-hole pairs. 


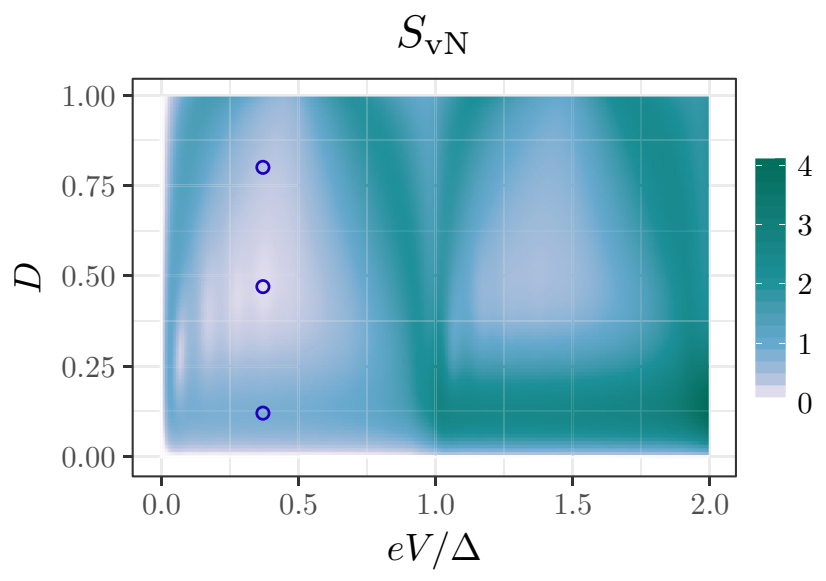

FIG. 18. Density plot of the electron-hole entanglement entropy at zero temperature for the mesoscopic capacitor operated with a square drive of frequency $f$ such that $\Delta / h f=20$ as a function of $\mathrm{eV} / \Delta$ and $D$.

A surprising feature are the substructures that appear within each shallow zone (see Fig. 19). At the time of this writing, we do not yet understand this fact. Further numerical exploration will be necessary, especially to see if the ratio $\Delta / h f$ plays a role in these substructures.

In order to understand more precisely the electron and hole entanglement properties described by this plot, we choose specific points for which we push the analysis further. The corresponding electronic Wigner distribution functions are plotted on Fig. 20.

\section{The Floquet-Bloch spectrum}

Let us review the Floquet-Bloch spectrum for the three points that are marked in Fig. 18. Figure 21 presents the corresponding bands as functions of the dimensionless quasienergy $\nu / 2 \pi f$ and orders them according to their averages, the $a=0$ band being one with the highest average.

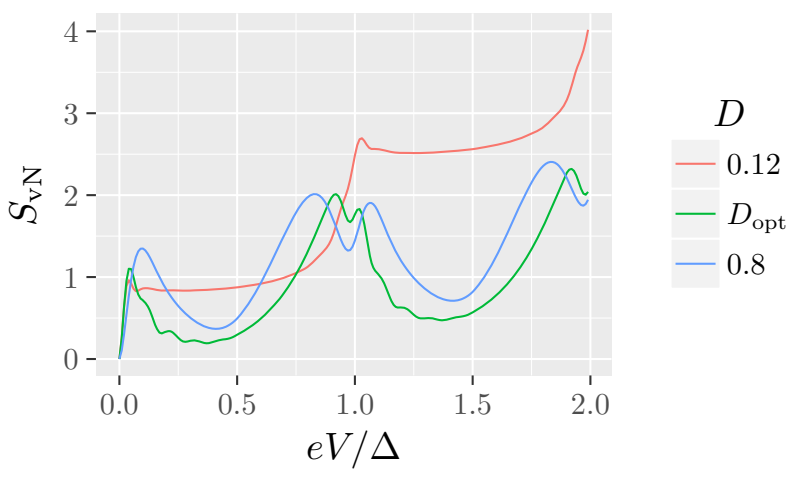

FIG. 19. Cuts of the entropy $S_{\mathrm{vN}}$ for a square voltage drive depicted on Fig. 18 as functions of $e V / \Delta$ for $D=0.12, D=$ $D_{\text {opt }}$, and $D=0.8$.

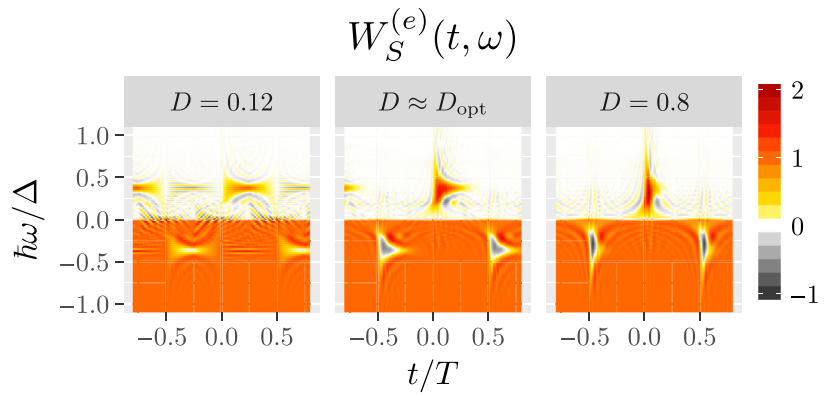

FIG. 20. Density plot of the full Wigner distribution function $W_{S}^{(e)}(t, \omega)$ for the square-voltage driven mesoscopic capacitor as a function of $t / T$ and $\hbar \omega / \Delta$ for the three selected points appearing on Fig. 18.

Opening the dot $(D=0.8$, right panel) leads to flat bands as expected since at $D=1$ it is really what is expected but we note that the eigenvalues are almost unity and that the $a=1$ band has value 0.02 , thus showing that we are departing from the ideal single-electron regime.

Closing the dot $(D=0.12$, left panel) mostly changes the shape of the $a=0$ band, which shows some curvature. Its average is equal to 0.57 , which shows that strong electron-hole coherences are expected.

\section{Electronic atoms of signals and coherences}

In order to get a clearer view of the electronic state emitted by the source, let us now extract the corresponding electronic atoms of signal. Figure 22 presents the electronic atoms of signal associated with the $a=0$ FloquetBloch band for the three operating points considered before.

As expected, the duration of each wave packet increases with decreasing $D$ reflecting the fact that the escaping time from the dot is longer at low QPC transparency. At the optimal value $D_{\text {opt }}$, we expect the source to emit a wave

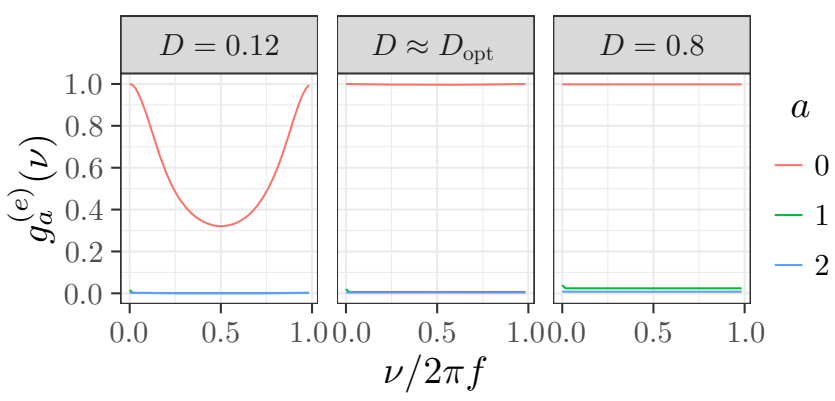

FIG. 21. Floquet-Bloch spectrum for the three selected points appearing on Fig. 18, in the case of a square-voltage mesoscopic capacitor. Only the first three bands are represented, all the other ones being even closer to zero. 


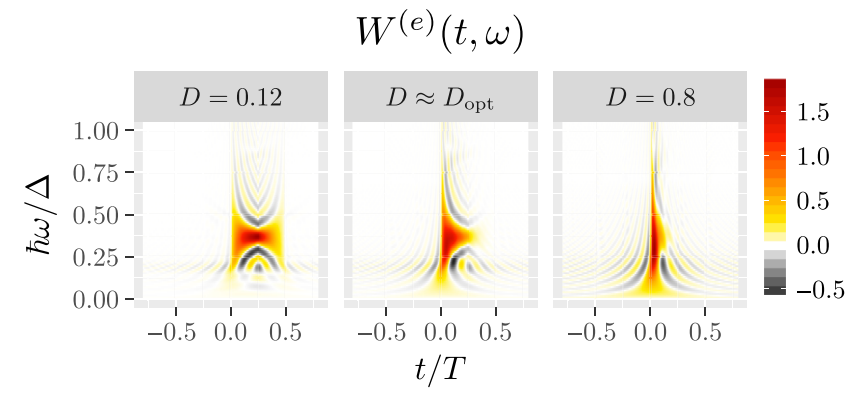

FIG. 22. Wigner distribution functions for the FloquetWannier electronic atoms of signal corresponding to the $a=0$ Floquet-Bloch band represented as a function of $t / T$ and $\hbar \omega / \Delta$ for the three operating points of Fig. 18 in the case of a square voltage drive.

packet of the form

$$
\widetilde{\varphi}_{e}(\omega)=\frac{\mathcal{N}_{e} H(\omega)}{\omega-\omega_{e}-i \gamma_{e} / 2}
$$

where $\mathcal{N}_{e}$ ensures normalization and $\gamma_{e}$ denotes the electron escape rate from the quantum dot, which is given by $\gamma_{e}=D \Delta / h(1-D / 2)[46,96]$. The square drive thus leads, as expected, to wave functions that are better separated from the Fermi sea than the ones emitted with a sine drive. These are better suited for studying electronic decoherence of wave packets with a given average energy since this can be adjusted via a dc voltage applied to the top gate of the dot in the mesoscopic capacitor [72]. On the other hand, the sine-drive wave packets may be relevant for metrological applications but discussing this would go beyond the scope of the present paper.

We note that for $D=0.12$, the electronic wave packet remains limited to the first half period $0 \lesssim t \lesssim T / 2$. At very low $D$, we expect this wave packet to be the projection on the space of single-particle states with positive energy of the dual of the Martin-Landauer wave packet, that is of an electronic wave function constant on a time interval.

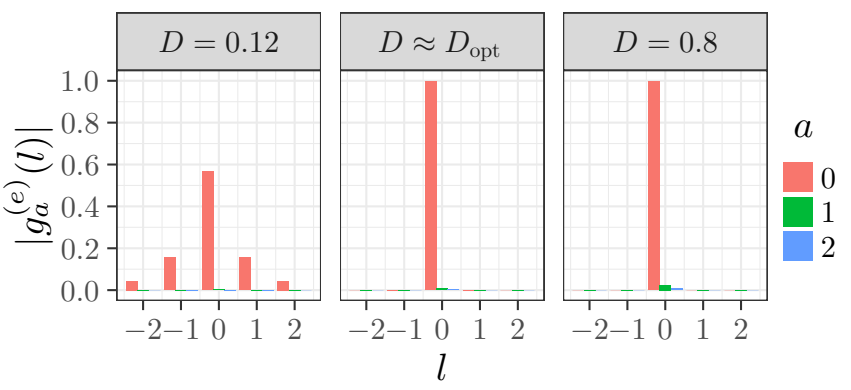

FIG. 23. Temporal coherences $p_{n}(\Delta l)$ between the electronic atoms of signal of the $a=0,1$, and 2 Floquet-Bloch bands given by Eq. (19) as a function of $\Delta l$ for the three operating points of Fig. 18 in the case of a square drive.
Since the bands are flat for $D=D_{\mathrm{opt}}$ and $D=0.8$, no interperiod coherence is expected as can be seen from the middle and right panels of Fig. 23. However, when closing the dot $(D \approx 0.12$, left panel), interperiod coherences for the electronic excitations start to unfold, an expected consequence of the delocalization of the emitted electronic excitations over more than a half period. This shows that the electronic coherence time is given by the electronic escape time which, in this case, exceeds the duration of an electronic atom of signal.

\section{APPENDIX H: WAVE FUNCTIONS WITHIN A LEVITON TRAIN}

We define a Levitonoid as a normalized wave function $\psi(t)$ such that their time translations by multiples of $T$ are mutually orthogonal and $\sum_{l \in \mathbb{Z}} \psi(t-l T) \psi^{*}\left(t^{\prime}-l T\right)$ is the excess electronic first-order coherence generated by a $T$ periodic train of Lorentzian pulses. As we see, this wave function is not unique but, in the case of a $T$-periodic Leviton train, an analytical expression for the minimally spread Levitonoids can be obtained.

\section{The Moskalets atoms of signal}

In a recent work [47], Moskalets has identified one possible Levitonoid as

$$
\psi(t)=\sqrt{\frac{\tau_{0}}{\pi}} \frac{1}{t-i \tau_{0}} \prod_{n=1}^{\infty} \frac{t+n T+i \tau_{0}}{t+n T-i \tau_{0}},
$$

where $T=1 / f$ is the period and $\tau_{0}$ is the typical time width of the excitation. This wave function has a spatial extension given by $\tau_{0}$.

To discuss its energy content, let us use the identity

$$
\Gamma(z)=\frac{1}{z} \prod_{n=1}^{\infty} \frac{\left(1+\frac{1}{n}\right)^{z}}{1+\frac{z}{n}}
$$

to rewrite the infinite product as a ratio of $\Gamma$ functions, up to a global phase

$$
\begin{aligned}
\frac{\Gamma\left[\left(t-i \tau_{0}\right) / T\right]}{\Gamma\left[\left(t+i \tau_{0}\right) / T\right]} & =\frac{t+i \tau_{0}}{t-i \tau_{0}} \prod_{n=1}^{\infty}\left(1+\frac{1}{n}\right)^{-2 i \tau_{0}} \\
& \times \prod_{n=1}^{\infty} \frac{t+n T+i \tau_{0}}{t+n T-i \tau_{0}}
\end{aligned}
$$

Then, up to a global phase factor, we can rewrite

$$
\psi(t)=\sqrt{\frac{\tau_{0}}{\pi}} \frac{1}{t+i \tau_{0}} \frac{\Gamma\left[\left(t-i \tau_{0}\right) / T\right]}{\Gamma\left[\left(t+i \tau_{0}\right) / T\right]} .
$$


Fourier transforming this wave packet gives, up to global phase factor

$$
\psi(\omega)=\frac{1}{\sqrt{\mathcal{N}}} H(\omega)\left(2 \sin \frac{\nu T}{2}\right)^{2 i \tau_{0} / T} e^{-\omega_{\mathrm{int} \tau_{0}}}
$$

where $\omega=\omega_{\text {int }}+v$, with $v \in\left[0,2 \pi f\left[\right.\right.$ and thus $\omega_{\text {int }}=$ $2 \pi f\lfloor\omega / 2 \pi f\rfloor$. Here, $\mathcal{N}=f / v_{F}\left(1-e^{-4 \pi \tau_{0} / T}\right)$ is a normalization factor and $H$ the Heaviside step function. We can then rewrite the wave function as a real part and a periodic phase:

$$
\psi(\omega)=\frac{1}{\sqrt{\mathcal{N}}} H(\omega) e^{i \theta(\omega)} e^{-\omega_{\mathrm{int}} \tau_{0}},
$$

with the phase satisfying the condition $\theta(\omega+2 \pi f)=$ $\theta(\omega)$. This expression shows that the electronic distribution function of this Levitonoid is the staircase approximation of an exponential decay, with step widths given by $2 \pi f$ as expected from $T$ periodicity. Note that the electronic distribution function does not depend on the phase $\theta(\omega)$.

\section{The minimally spread atoms of signal}

The Moskalets Levitonoids having a spreading $\tau_{0}$, they are naturally expected to be among the minimally spread atoms of signals when $f \tau_{0} \ll 1$, that is when the Leviton spacing is large compared to their duration. But in the opposite limit $f \tau_{0} \gtrsim 1$, this is certainly not the case. Let us search for other Levitonoids that could be spread over the period $T$ and clarify the relation between our algorithm and Moskalets work [47].

If a quantum electrical current has a time-reversal symmetry, which is the case for a Leviton train, then there must be a set of Wannier wave functions that possess this symmetry. Consequently, there is a set of real-valued Wannier functions in the frequency domain: $\varphi_{\text {Lev }}(\omega) \in \mathbb{R}$. Assuming that $\varphi_{\text {Lev }}(\omega) \geq 0$, the time-spreading minimization problem becomes trivial and we find that, up to time translation by $T$, the minimal wave functions are the ones that possess the time-reversal symmetry.

In the case of Levitonoids, Eq. (H6) shows that the wave functions can be written as the product of a real part and a phase part, the phase part being periodic in time. Thus the minimization of Eq. (C8) is realized when the minimal wave function has a constant phase. Setting this global phase to zero, we have the following wave function:

$$
\varphi_{\mathrm{Lev}}(\omega)=\frac{1}{\sqrt{\mathcal{N}}} H(\omega) e^{-\omega_{\mathrm{int}} \tau_{0}}
$$

which is time-reversal invariant. In this case, the current of one pulse is different from the current of one Leviton of duration $\tau_{0}$. The time-domain expression for this wave packet is

$$
\varphi_{\mathrm{Lev}}(t)=\frac{i}{\sqrt{\mathcal{N}^{\prime}}} \frac{1}{t} \frac{1-e^{-2 i \pi f t}}{1-e^{-2 \pi f\left(\tau_{0}+i t\right)}}
$$

The corresponding average current $i_{\text {Lev }}(t)=-v_{F}|\varphi(t)|^{2}$ (in units of $-e$ ) is then

$$
i_{\text {Lev }}(t) \propto \frac{\operatorname{sinc}^{2}(\pi f t)}{1-\cos (2 \pi f t) / \cosh \left(2 \pi f \tau_{0}\right)} .
$$

The overlap between this wave packet and a unique Leviton $\varphi_{1}$ is given by

$$
\left|\left\langle\varphi_{\text {Lev }} \mid \varphi_{1}\right\rangle\right|^{2}=\frac{1}{\pi f \tau_{0}} \frac{1-e^{-2 \pi f \tau_{0}}}{1+e^{-2 \pi f \tau_{0}}} .
$$

The behavior when $f \tau_{0} \ll 1$ is approached by

$$
\left|\left\langle\varphi_{\text {Lev }} \mid \varphi_{1}\right\rangle\right|^{2} \simeq 1-\frac{\left(\pi f \tau_{0}\right)^{2}}{6}
$$

making the Leviton approximation a fairly good approximation in this case. When $f \tau_{0} \gg 1$, we have a rather slow decay:

$$
\left|\left\langle\varphi_{\text {Lev }} \mid \varphi_{1}\right\rangle\right|^{2} \simeq \frac{1}{\pi f \tau_{0}} .
$$

\section{Obtention from Martin-Landauer's wave packets}

As noticed in Ref. [68, Appendix 5], at zero temperature, the effect of a $T$-periodic classical drive $V_{d}$ is to reshuffle the Martin-Landauer wave packets associated with a given period through a unitary transformation of the following form:

$$
\left|\mathrm{ML}_{n, l}\right\rangle \mapsto \sum_{k \in \mathbb{Z}} p_{k}\left[V_{d}\right]\left|\mathrm{ML}_{n+k, l}\right\rangle
$$

in which $\left|\mathrm{ML}_{n, l}\right\rangle$ denotes the time translated to period $l$ of the single-particle state defined by Eq. (24) and $p_{k}\left[V_{d}\right]$ is the photoassisted transition amplitude associated with this drive. In the case of a Leviton train, the photoassisted amplitudes $p_{k}$ are known and given by [68]

$$
\begin{aligned}
p_{k<-1} & =0, \\
p_{-1} & =e^{-2 \pi f \tau_{0}}, \\
p_{k \geq 0} & =\left(1-e^{-4 \pi f \tau_{0}}\right) e^{-2 \pi k f \tau_{0}} .
\end{aligned}
$$

Consequently, introducing $\beta=e^{-2 \pi f \tau_{0}}$ for compacity, for all states with the Fermi sea $(n \leq-1)$ :

$$
\begin{aligned}
\left|\mathrm{ML}_{-n, l}\right\rangle \mapsto & -\beta\left|\mathrm{ML}_{-(n+1), l}\right\rangle \\
& +\left(1-\beta^{2}\right) \sum_{k=1}^{n} \beta^{n-k}\left|\mathrm{ML}_{-k, l}\right\rangle
\end{aligned}
$$




$$
+\left(1-\beta^{2}\right) \beta^{n} \sum_{q=0}^{+\infty} \beta^{q}\left|\mathrm{ML}_{q, l}\right\rangle
$$

in which we separate what remains into the Fermi sea (first line) from what emerges from the Fermi sea (second line). This shows that, as expected, the projection of the single-particle scattering from the space generated by the Martin-Landauer wave packets of negative energy and given period onto the space of state generated by the ones of positive energy is of rank one. This is sufficient to obtain an electronic atom of signal for the Leviton associated with the period $l$ is

$$
\left|\operatorname{Lev}_{l}\right\rangle=\sqrt{1-\beta^{2}} \sum_{p=0}^{+\infty} \beta^{p}\left|\mathrm{ML}_{p, l}\right\rangle .
$$

Since the Martin-Landauer wave packet $\mathrm{ML}_{n, l=0}$ has a nonzero constant wave function over $2 \pi n f \leq \omega<$ $2 \pi(n+1) f$ and zero for $\omega \geq 2 \pi(n+1) f$ or $\omega<2 \pi n f$, the wave function of $\left|\mathrm{Lev}_{1}\right\rangle$ in the frequency domain is exactly given by Eq. (H7), therefore, showing that it is indeed the minimally spread Levitonoid!

\section{APPENDIX I: RANDOM EMISSION}

\section{Expression in the frequency domain}

Let us start from the excess coherence of a random train of $-e$ charge Lorentzian pulse

$$
\begin{aligned}
& \Delta \mathcal{G}^{(e)}(t+\tau / 2, t-\tau / 2) \\
& \quad=\mathcal{G}_{F}^{(e)}(\tau) \frac{\cos \left[2 \pi \theta_{p}(f \tau)\right]-\cos \left[2 \pi f\left(\tau / 2-i \tau_{0}\right)\right]}{\cos \left[2 \pi f\left(\tau / 2-i \tau_{0}\right)\right]-\cos (2 \pi f t)} .
\end{aligned}
$$

This expression is periodic in $t$ with a period $T=1 / f$. The $n$th harmonics $A_{n}(\tau)$ of its Fourier series expansion is then given by

$$
\begin{aligned}
A_{n}(\tau)= & -i \mathcal{G}_{F}(\tau) \frac{\cos \left[2 \pi \theta_{p}(f \tau)\right]-\cos \left[2 \pi f\left(\tau / 2-i \tau_{0}\right)\right]}{\sin \left[2 \pi f\left(\tau / 2-i \tau_{0}\right)\right]} \\
& \times e^{-2 \pi|n| f \tau_{0}} e^{-i \pi|n| f \tau} .
\end{aligned}
$$

Performing the Fourier transform along the variable $\tau$ leads to the energy representation of the first-order coherence. Let us notice that the Fourier transform is found to be zero when $\omega<\pi|n|$, meaning that the first-order coherence is nonzero only in the electronic quadrant, as expected. By looking at the electronic quadrant, we derive

$$
\mathcal{G}_{++, n}^{(e)}(\omega)=H(\omega-\pi f|n|) e^{-2 \omega \tau_{0}} \mathcal{F}_{p}(\omega+\pi n f),
$$

where $\mathcal{F}_{p}(\omega)$ is a $2 \pi f$-periodic real-valued function defined by the sum

$$
\mathcal{F}_{p}(\omega)=\frac{i}{\pi} \sum_{k \in \mathbb{Z}} \frac{\cos \left[\chi_{p}(k)\right]-1}{k+2 i f \tau_{0}} e^{i \omega k T},
$$

where $\chi_{p}(x)=2 \pi \theta_{p}\left(2 i f \tau_{0}+k\right)-\pi k$. In order to evaluate numerically this expression, we decompose $\mathcal{F}_{p}=$ $\mathcal{F}_{p}^{\text {(sing) }}+\mathcal{F}_{p}^{\text {(reg) }}$, where $\mathcal{F}_{p}^{\text {(sing) }}$ contains singularities due to the slow decay (approximately $1 / k$ ) at infinity. We have

$$
\mathcal{F}_{p}^{(\text {sing })}(\omega)=\frac{i}{\pi} \sum_{k \in \mathbb{Z}} \frac{\cosh \left(4 \pi p \tau_{0}\right)-1}{k+2 i f \tau_{0}} e^{i \omega k T},
$$

$$
\mathcal{F}_{p}^{(\mathrm{reg})}(\omega)=\frac{i}{\pi} \sum_{k \in \mathbb{Z}} \frac{\cos \left[\chi_{p}(k)\right]-\cosh \left(4 \pi p \tau_{0}\right)}{k+2 i f \tau_{0}} e^{i \omega k T}
$$

The singular part then contributes to the single-electron coherence by

$$
\mathcal{G}_{+, n}^{(e, \text { sing })}(\omega)=4 H(\omega-\pi f|n|) \frac{\sinh ^{2}\left(2 \pi f p \tau_{0}\right)}{e^{4 \pi f \tau_{0}}-1} e^{-2 \omega_{n} \tau_{0}},
$$

where $\omega_{n}=2 \pi f\lfloor\omega / 2 \pi f\rfloor$ if $n$ is even and $\omega_{n}=$ $2 \pi f\lfloor\omega / 2 \pi f-1 / 2\rfloor+\pi f$ if $n$ is odd. The regular part can then be evaluated numerically by direct summation. To be more precise, we can bound the truncation error on the regular part, when using $K$ terms on the sum. Using an asymptotic expansion, we find that the error scales as

$$
\epsilon=16 p(1-p)\left(f \tau_{0}\right)^{2} \frac{\sinh \left(4 \pi p f \tau_{0}\right)}{K} .
$$

On top of the polynomial scaling, we notice an exponential scaling in $p$ and in $f \tau_{0}$. Actually, as we will see later, we can use a symmetry in $p \leftrightarrow 1-p$, to ensure that all the computations are done at $p \leq 1 / 2$. Using this, it is reasonable to compute the sum for $\tau \lesssim 1$.

When $p=1$, the regular contribution cancels out, and we find the usual expression for a Leviton train. When $p \rightarrow 0$, it is the singular contribution that disappears first (scaling as $p^{2}$ ) leaving only the regular contribution (scaling as $p)$. When $p \ll \min \left(1, f \tau_{0}\right), \mathcal{F}_{p}(\omega)=4 \pi f p \tau_{0}$ is constant. Each harmonics of the first-order coherence is thus an exponential decay

$$
\mathcal{G}_{++, n}^{(e)}(\omega)=4 \pi f p H(\omega-\pi|n| f) \tau_{0} e^{-2 \omega \tau_{0}} .
$$




\section{Electronic atoms of signal}

The electronic atoms of signal describing the random train's first-order coherence are obtained by following the method presented in Sec. III. The projection of the singleelectron coherence on the electronic quadrant has nonzero matrix elements only for $\omega_{ \pm}=v+2 \pi n_{ \pm} f$ in which $0 \leq$ $v<2 \pi f$ and $n_{ \pm}$are positive integers. This corresponds to $\omega=\left(\omega_{+}+\omega_{-}\right) / 2=v+\pi f\left(n_{+}+n_{-}\right)$and $\Omega=\omega_{+}-$ $\omega_{-}=2 \pi\left(n_{+}-n_{-}\right) f$. Then, using Eq. (I3) and the periodicity of $\mathcal{F}_{p}(\Omega)$ in $\Omega \rightarrow \Omega+2 \pi f$, the excess first-order electronic coherence can be rewritten as

$$
\begin{gathered}
\Delta_{0} \mathbf{G}^{(e)}=\int_{0}^{2 \pi f} e^{-2 v \tau_{0}} \mathcal{F}_{p}(v) \mathbf{M}(v) \frac{d v}{2 \pi f}, \\
\mathbf{M}(v)=\sum_{n_{ \pm} \in \mathbb{N}} e^{-2 \pi\left(n_{+}+n-\right) f \tau_{0}}\left|\nu+2 \pi f n_{+}\right\rangle\left\langle\nu+2 \pi f n_{-}\right| .
\end{gathered}
$$

We thus have to diagonalize the operator $\mathbf{M}(v)$ for each $0 \leq v<2 \pi f$. We have already seen how to diagonalize it: for each $v$, this operator has rank one and its eigenvector is the one obtained in Eq. (H16). This immediately shows that we have the same Floquet-Bloch states (only one band here) and, therefore, the same electronic atoms of signals than for the periodic Leviton train ( $p=1$, Appendices $\mathrm{H} 2$ and $\mathrm{H} 3$ ).

Only the eigenvalue is modified by randomness:

$$
g^{(e)}(v)=\left(1-e^{-4 \pi f \tau_{0}}\right)^{-1} e^{-2 v \tau_{0}} \mathcal{F}_{p}(\nu)
$$

When $p=1$, the periodic Leviton train with its flat band with $g^{(e)}(v=1)$ is recovered but for $p<1$, the band is not flat anymore. This means that for $p<1$, the emission probability of emission of the Levitonoid is lower than unity but this also leads to interperiod coherences between the Levitonoids. A numerical evaluation of the rhs of Eq. (I11) is shown on Fig. 24, which confirms these features.

Finally, using this last expression, we can rewrite the zeroth-harmonic of the first-order coherence as

$$
\mathcal{G}_{0}^{(e)}(\omega)=\left(1-e^{-4 \pi f \tau_{0}}\right) e^{-2 \pi f\left\lfloor\frac{\omega}{2 \pi f}\right\rfloor \tau_{0}} g^{(e)}(\omega) .
$$

By performing the inverse Fourier transform on this expression, we can thus express the coherences between the time-shifted Levitonoids as $g^{(e)}(l)=T A_{0}(-l T)$. Thus, we find

$$
\begin{gathered}
g^{(e)}(l=0)=p, \\
g^{(e)}(l \neq 0)=\frac{i}{2 \pi l} \frac{\cosh \left(2 \pi f \tau_{0}\right)-(-1)^{l} \cos \left[2 \pi \theta_{p}(-l)\right]}{\sinh 2 \pi f \tau_{0}} .
\end{gathered}
$$

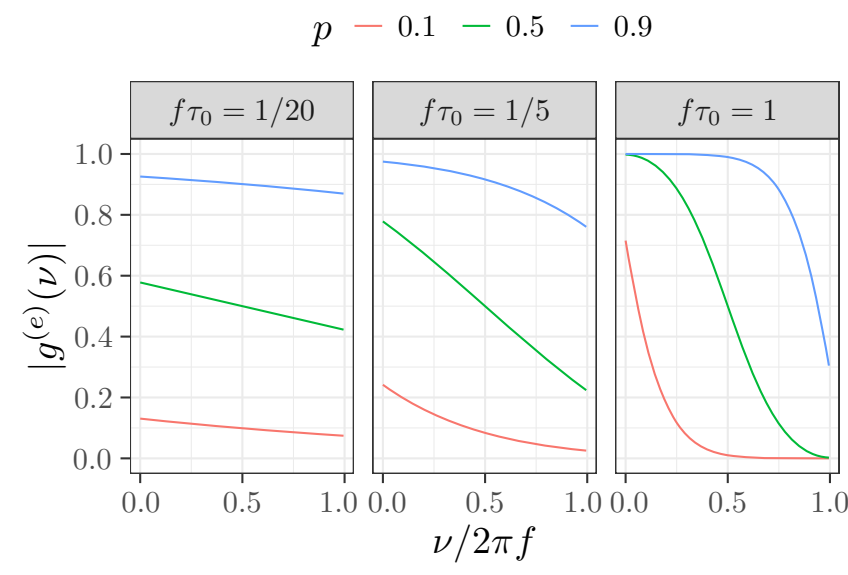

FIG. 24. Floquet-Bloch spectrum for the random train of Lorentzian pulses (it has only one band) for width $f \tau_{0}=1 / 10$, $1 / 5$, and 1 for three different values of $p(1 / 10,1 / 2$, and $9 / 10)$. Bands are more and more flat when decreasing $f \tau_{0}$ as expected since, in this limit, one recovers the random emission of a single electronic atom of signal per period with probability $p$. When $p$ goes to unity, we see the band getting closer to a flat band at value one, which corresponds to the $T$-periodic train of Levitons.

This expression satisfies $g_{p}^{(e)}(l \neq 0)=-g_{1-p}^{(e)}(l)^{*}$, which leads to the symmetry property

$$
g_{p}^{(e)}(\omega)=1-g_{1-p}^{(e)}(-\omega) .
$$

We use this symmetry to perform all the numerical computations at $p \leq 1 / 2$.

\section{Resumming interperiod coherences}

In Appendix I2, we see that it is possible to decompose the signal on Levitonoids. In a sense, Levitonoids are proper atoms of signals, because they do not overlap when they are time shifted by an integer number of periods. However, when $p<1$, coherences appear between time shifted Levitonoids.

Remarkably, is it possible to express the excess singleelectron coherence $\mathbf{G}_{R_{p}}^{(e)}$ in terms of wave functions associated with each period (and obtained by applying the translation operator $\mathbf{T}_{T}$ ), each of them emitted with probability $p$, without any interperiod coherence:

$$
\Delta_{0} \mathbf{G}^{(e)}=p \sum_{l \in \mathbb{Z}}\left|\mathrm{Gla}_{p, l}\right\rangle\left\langle\mathrm{Gla}_{p, l}\right|
$$

in which $\left|\mathrm{Gla}_{p, l}\right\rangle=\mathbf{T}_{T}^{l}\left|\mathrm{Gla}_{p}\right\rangle$ is obtained by a time translation by $l$ periods from

$$
\left|\mathrm{Gla}_{p}\right\rangle=\int_{0}^{+\infty} \sqrt{\frac{1-e^{-4 \pi f \tau_{0}}}{p f}} e^{-\omega_{\mathrm{int}} \tau_{0}} \sqrt{g^{(e)}(\omega)}|\omega\rangle d \omega
$$


in which $\omega_{\text {int }}=2 \pi f\lfloor\omega / 2 \pi f\rfloor$. We call such a singleparticle state a $p$-Glattlion in reference to [45]. However, the overlap of adjacent $p$-Glattlions is nonvanishing and is related to the electronic coherences between two different periods in the minimally spread Levitonoids:

$$
p\left\langle\operatorname{Gla}_{p, 0} \mid \operatorname{Gla}_{p, l}\right\rangle=\int_{0}^{2 \pi f} g^{(e)}(v) e^{i v l T} \frac{d \nu}{2 \pi f}=g^{(e)}(l)
$$

In other words, the coherences between the Levitonoids are given by the overlap between time-shifted $p$-Glattlions, which, therefore, cannot be considered as electronic atoms of signals. Despite this nonvanishing overlap, it is quite remarkable that the excess single-electron coherence for the random train of Levitons can be rewritten in such a simple form.

Finally, it is worth noting that the 1-Glattlion is nothing else than the minimal Levitonoid. On the other hand, when $p \rightarrow 0$, a $p$-Glattlion becomes close to isolated Levitons. This is related to the fact that when $p$ is small, pulses are emitted as if they are isolated: the probability for a single pulse to be separated by a distance at least $k$ from the previous and the next one is $(1-p)^{2 k}$, which goes to unity when $p \rightarrow 0$. As such, they do not exhibit Pauli exclusion principle between pulses.

[1] G. Fève, A. Mahé, J. Berroir, T. Kontos, B. Plaçais, D. C. Glattli, A. Cavanna, B. Etienne, and Y. Jin, An ondemand single electron source, Science 316, 1169 (2007).

[2] C. Leicht, P. Mirovsky, B. Kaestner, F. Hols, V. Kashcheyevs, E. Kurganova, U. Zeitler, T. Weimann, K. Pierz, and H. Schumacher, Generation of energy selective excitations in quantum Hall edge states, Semicond. Sci. Technol. 26, 055010 (2011).

[3] J. Dubois, T. Jullien, F. Portier, P. Roche, A. Cavanna, Y. Jin, W. Wegscheider, P. Roulleau, and D. C. Glattli, Minimal excitation states for electron quantum optics using Levitons, Nature 502, 659 (2013).

[4] M. Kataoka, N. Johnson, C. Emary, P. See, J. P. Griffiths, G. A. C. Jones, I. Farrer, D. A. Ritchie, M. Pepper, and T. J. B. M. Janssen, Time-Of-Flight Measurements of SingleElectron Wave Packets in Quantum Hall Edge States, Phys. Rev. Lett. 116, 126803 (2016).

[5] J. D. Fletcher, P. See, H. Howe, M. Pepper, S. P. Giblin, J. P. Griffiths, G. A. C. Jones, I. Farrer, D. A. Ritchie, T. J. B. M. Janssen, and M. Kataoka, Clock-Controlled Emission of Single-Electron Wave Packets in a Solid-State Circuit, Phys. Rev. Lett. 111, 216807 (2013).

[6] S. Hermelin, S. Takada, M. Yamamoto, S. Tarucha, A. Wieck, L. Saminadayar, C. Bäuerle, and T. Meunier, Electrons surfing on a sound wave as a platform for quantum optics with flying electrons, Nature 477, 435 (2011).

[7] J. Gabelli and B. Reulet, Shaping a time-dependent excitation to control the electron distribution function: Noise minimization in a tunnel junction, Phys. Rev. B 87, 075403 (2013).

[8] E. Bocquillon, V. Freulon, F. Parmentier, J. Berroir, B. Plaçais, C. Wahl, J. Rech, T. Jonckheere, T. Martin, C. Grenier, D. Ferraro, P. Degiovanni, and G. Fève, Electron quantum optics in ballistic chiral conductors, Ann. Phys. (Berlin) 526, 1 (2014).

[9] R. Bisognin, A. Marguerite, B. Roussel, M. Kumar, C. Cabart, C. Chapdelaine, A. Mohammad-Djafari, J. M. Berroir, E. Bocquillon, B. Plaçais, A. Cavanna, U. Gennser, Y. Jin, P. Degiovanni, and G. Fève, Quantum tomography of electrical currents, Nat. Commun. 10, 3379 (2019).

[10] A. Bertoni, P. Bordone, R. Brunetti, C. Jacoboni, and S. Reggiani, Quantum Logic Gates Based on Coherent Electron Transport in Quantum Wires, Phys. Rev. Lett. 84, 5912 (2000).

[11] R. Ionicioiu, G. Amaratunga, and F. Udrea, Quantum computation with ballistic electrons, Int. J. Mod. Phys. B 15, 125 (2001).

[12] A. Bertoni, Perspectives on solid-state flying qubits, J. Comput. Electron. 6, 67 (2007).

[13] T. Zibold, P. Vogl, and A. Bertoni, Theory of semiconductor quantum-wire-based single- and two-qubit gates, Phys. Rev. B 76, 195301 (2007).

[14] A. Bertoni, in Encyclopedia of Complexity and Systems Science, edited by R. A. Meyers (Springer, New York, NY, 2009), p. 1011.

[15] M. Yamamoto, S. Takada, C. Bäuerle, K. Watanabe, A. D. Wieck, and S. Tarucha, Electrical control of a solid-state flying qubit, Nat. Nanotechnol. 7, 247 (2012).

[16] B. Bertrand, S. Hermelin, S. Takada, M. Yamamoto, S. Tarucha, A. Ludwig, A. D. Wieck, C. Bäuerle, and T. Meunier, Fast spin information transfer between distant quantum dots using individual electrons, Nat. Nanotechnol. 11, 672 (2016).

[17] H. Flentje, P. A. Mortemousque, R. Thalineau, A. Ludwig, A. D. Wieck, C. Bäuerle, and T. Meunier, Coherent longdistance displacement of individual electron spins, Nat. Commun. 8, 501 (2017).

[18] C. W. J. Beenakker, D. P. DiVincenzo, C. Emary, and M. Kindermann, Charge Detection Enables Free-Electron Quantum Computation, Phys. Rev. Lett. 93, 020501 (2004).

[19] C. H. W. Barnes, J. M. Shilton, and A. M. Robinson, Quantum computation using electrons trapped by surface acoustic waves, Phys. Rev. B 62, 8410 (2000).

[20] S. Takada, H. Edlbauer, H. V. Lepage, J. Wang, P.-A. Mortemousque, G. Georgiou, C. H. W. Barnes, C. J. B. Ford, M. Yuan, P. V. Santos, X. Waintal, A. Ludwig, A. D. Wieck, M. Urdampilleta, T. Meunier, and C. Bäuerle, Sound-driven single-electron transfer in a circuit of coupled quantum rails, Nat. Commun. 10, 4557 (2019).

[21] R. Glauber, The quantum theory of optical coherence, Phys. Rev. 130, 2529 (1963).

[22] G. C. Wick, A. S. Wightman, and E. P. Wigner, The intrinsic parity of elementary particles, Phys. Rev. 88, 101 (1952).

[23] N. Friis, Reasonable fermionic quantum information theories require relativity, New J. Phys. 18, 033014 (2016).

[24] M. Johansson, Comment on 'Reasonable fermionic quantum information theories require relativity', arXiv: 1610. 00539 (2016). 
[25] C. Grenier, R. Hervé, G. Fève, and P. Degiovanni, Electron quantum optics in quantum Hall edge channels, Mod. Phys. Lett. B 25, 1053 (2011). Proceedings of the Statphys 24 satellite meeting "International Conference on Frustrated Spin Systems, Cold Atoms and Nanomaterials" (Hanoi, 14-16 July 2010).

[26] G. Haack, M. Moskalets, and M. Büttiker, Glauber coherence of single electron sources, Phys. Rev. B 87, 201302(R) (2013).

[27] M. Moskalets, Two-electron state from the Floquet scattering matrix perspective, Phys. Rev. B 89, 045402 (2014).

[28] E. Thibierge, D. Ferraro, B. Roussel, C. Cabart, A. Marguerite, G. Fève, and P. Degiovanni, Two-electron coherence and its measurement in electron quantum optics, Phys. Rev. B 93, 081302(R) (2016).

[29] B. Roussel, C. Cabart, G. Fève, E. Thibierge, and P. Degiovanni, Electron quantum optics as quantum signal processing, Phys. Status Solidi B 254, 16000621 (2017).

[30] G. Haack, M. Moskalets, J. Splettstoesser, and M. Büttiker, Coherence of single-electron sources from Mach-Zehnder interferometry, Phys. Rev. B 84, 081303(R) (2011).

[31] S. Ol'khovskaya, J. Splettstoesser, M. Moskalets, and M. Büttiker, Shot Noise of a Mesoscopic Two-Particle Collider, Phys. Rev. Lett. 101, 166802 (2008).

[32] J. Splettstoesser, M. Moskalets, and M. Büttiker, TwoParticle Nonlocal Aharonov-Bohm Effect from two SingleParticle Emitters, Phys. Rev. Lett. 103, 076804 (2009).

[33] E. Bocquillon, V. Freulon, J. Berroir, P. Degiovanni, B. Plaçais, A. Cavanna, Y. Jin, and G. Fève, Coherence and indistinguability of single electrons emitted by independent sources, Science 339, 1054 (2013).

[34] T. Jullien, P. Roulleau, P. Roche, A. Cavanna, Y. Jin, and D. C. Glattli, Quantum tomography of an electron, Nature 514, 603 (2014).

[35] J. D. Fletcher, N. Johnson, E. Locane, P. See, J. P. Griffiths, I. Farrer, D. A. Ritchie, P. W. Brouwer, V. Kashcheyevs, and M. Kataoka, Continuous-variable tomography of solitary electrons, Nat. Commun. 10, 5298 (2019).

[36] F. Bloch, Über die quantenmechanik der elektronen in kristallgittern, Z. Phys. 52, 555 (1929).

[37] G. Wannier, The structure of electronic excitation levels in insulating crystal, Phys. Rev. 52, 191 (1937).

[38] C. Grenier, R. Hervé, E. Bocquillon, F. Parmentier, B. Plaçais, J. Berroir, G. Fève, and P. Degiovanni, Single electron quantum tomography in quantum Hall edge channels, New J. Phys. 13, 093007 (2011).

[39] R. Bisognin, H. Bartolomei, M. Kumar, I. Safi, J.-M. Berroir, E. Bocquillon, B. Plaçais, A. Cavanna, Y. Gennser, Y. Jin, and G. Fève, Microwave photons emitted by fractionally charged quasiparticles, Nat. Commun. 10, 1708 (2019).

[40] D. Ferraro, A. Feller, A. Ghibaudo, E. Thibierge, E. Bocquillon, G. Fève, C. Grenier, and P. Degiovanni, Wigner function approach to single electron coherence in quantum Hall edge channels, Phys. Rev. B 88, 205303 (2013).

[41] T. Martin and R. Landauer, Wave-packet approach to noise in multichannel mesoscopic systems, Phys. Rev. B 45, 1742 (1992).
[42] F. Hassler, M. V. Suslov, G. M. Graf, M. V. Lebedev, G. B. Lesovik, and G. Blatter, Wave-packet formalism of full counting statistics, Phys. Rev. B 78, 165330 (2008).

[43] J. Moura, What is signal processing?, IEEE Signal Process. Mag. 26, 6 (2009).

[44] Y. Yin, Quasiparticle states of on-demand coherent electron sources, J. Phys.: Condens. Matter 31, 245301 (2019).

[45] D. C. Glattli and P. Roulleau, Pseudorandom binary injection of Levitons for electron quantum optics, Phys. Rev. B 97, 125407 (2018).

[46] A. Mahé, F. Parmentier, G. Fève, J. Berroir, T. Kontos, A. Cavanna, B. Etienne, Y. Jin, D. C. Glattli, and B. Plaçais, Subnanosecond single electron source in the time domain, J. Low Temp. Phys. 153, 339 (2008).

[47] M. Moskalets, First-order correlation function of a stream of single-electron wave-packets, Phys. Rev. B 91, 195431 (2015).

[48] C. Wahl, J. Rech, T. Jonckheere, and T. Martin, Interactions and Charge Fractionalization in an Electronic HongOu-Mandel Interferometer, Phys. Rev. Lett. 112, 046802 (2014).

[49] D. Ferraro, B. Roussel, C. Cabart, E. Thibierge, G. Fève, C. Grenier, and P. Degiovanni, Real-Time Decoherence of Landau and Levitov Quasiparticles in Quantum Hall Edge Channels, Phys. Rev. Lett. 113, 166403 (2014).

[50] A. Marguerite, C. Cabart, C. Wahl, B. Roussel, V. Freulon, D. Ferraro, C. Grenier, J. M. Berroir, B. Placais, T. Jonckheere, J. Rech, T. Martin, P. Degiovanni, A. Cavanna, Y. Jin, and G. Fève, Decoherence and relaxation of a single electron in a one-dimensional conductor, Phys. Rev. B 94, 115311 (2016).

[51] C. Cabart, B. Roussel, G. Fève, and P. Degiovanni, Taming electronic decoherence in 1d chiral ballistic quantum conductors, Phys. Rev. B 98, 155302 (2018).

[52] G. Floquet, Sur les équations différentielles linéaires à coefficients périodiques, Ann. Ecole Norm. Sup. 12, 47 (1883).

[53] N. Marzari, A. A. Mostofi, J. R. Yates, I. Souza, and D. Vanderbilt, Maximally localized Wannier functions: Theory and applications, Rev. Mod. Phys. 84, 1419 (2012).

[54] E. Locane, P. W. Brouwer, and V. Kashcheyevs, Timeenergy filtering of single electrons in ballistic waveguides, New J. Phys. 21, 093042 (2019).

[55] More precisely, $\mathcal{V}_{d}(t)=\int_{-\infty}^{x}\left(\partial_{x} U\right)\left(x, t-x / v_{F}\right) d x$ in which $U(x, t)=V_{d}(t) u(x)$ denotes the voltage seen by the electrons as they fly across the QPC $[u(x)$ being nonzero close to the constriction of the QPC and rapidly vanishing away from it].

[56] G. Yamahata, S. Ryu, N. Johnson, H. S. Sim, A. Fujiwara, and M. Kataoka, Picosecond coherent electron motion in a silicon single-electron source, Nat. Nanotechnol. 14, 1019 (2019).

[57] T. Wenz, F. Hohls, X. Jehl, M. Sanquer, S. Barraud, J. Klochan, G. Barinovs, and V. Kashcheyevs, Dopantcontrolled single-electron pumping through a metallic island, Appl. Phys. Lett. 108, 213107 (2016).

[58] C. Altimiras, H. le Sueur, U. Gennser, A. Cavanna, D. Mailly, and F. Pierre, Non-equilibrium edge channel spectroscopy in the integer quantum Hall regime, Nat. Phys. 6, 34 (2010). 
[59] X. K. Yue and Y. Yin, Normal and anomalous electron-hole pairs in a quantum conductor driven by a voltage pulse, Phys. Rev. B 99, 235431 (2019).

[60] M. Vanević, Y. V. Nazarov, and W. Belzig, Elementary charge-transfer processes in mesoscopic conductors, Phys. Rev. B 78, 245308 (2008).

[61] M. Vanević, J. Gabelli, W. Belzig, and B. Reulet, Electron and electron-hole quasiparticle states in a driven quantum contact, Phys. Rev. B 93, 041416(R) (2016).

[62] M. Vanević, Y. V. Nazarov, and W. Belzig, Electron and electron-hole excitations in a driven Fermi sea, Phys. Status Solidi B 254, 1600551 (2017).

[63] L. Amico, R. Fazio, A. Osterloh, and V. Vedral, Entanglement in many-body systems, Rev. Mod. Phys. 80, 517 (2008).

[64] C. H. Bennett, H. J. Bernstein, S. Popescu, and B. Schumacher, Concentrating partial entanglement by local operations, Phys. Rev. A 53, 2046 (1996).

[65] H. Li and F. D. M. Haldane, Entanglement Spectrum as a Generalization of Entanglement Entropy: Identification of Topological Order in Non-Abelian Fractional Quantum Hall Effect States, Phys. Rev. Lett. 101, 010504 (2008).

[66] H. F. Song, S. Rachel, C. Flindt, I. Klich, N. Laflorencie, and K. Le Hur, Bipartite fluctuations as a probe of manybody entanglement, Phys. Rev. B 85, 035409 (2012).

[67] I. Klich, Lower entropy bounds and particle number fluctuations in a Fermi sea, J. Phys. A 39, L85 (2006).

[68] J. Dubois, T. Jullien, C. Grenier, P. Degiovanni, P. Roulleau, and D. C. Glattli, Integer and fractional charge lorentzian voltage pulses analyzed in the framework of photon-assisted shot noise, Phys. Rev. B 88, 085301 (2013).

[69] L. Levitov, H. Lee, and G. Lesovik, Electron counting statistics and coherent states of electric current, J. Math. Phys. 37, 4845 (1996).

[70] M. Moskalets, Scattering Matrix Approach to NonStationary Quantum Transport (Imperial College Press, London, 2011).

[71] M. Moskalets, P. Samuelsson, and M. Büttiker, Quantized Dynamics of a Coherent Capacitor, Phys. Rev. Lett. 100, 086601 (2008).

[72] A. Marguerite, E. Bocquillon, J.-M. Berroir, B. Plaçais, P. Degiovanni, and G. Fève, Two-particle interferometry in quantum Hall edge channels, Phys. Status Solidi B 254, 1600618 (2017).

[73] M. Misiorny, G. Fève, and J. Splettstoesser, Shaping charge excitations in chiral edge states with a time-dependent gate voltage, Phys. Rev. B 97, 075426 (2018).

[74] C. Grenier, J. Dubois, T. Jullien, P. Roulleau, D. C. Glattli, and P. Degiovanni, Fractionalization of minimal excitations in integer quantum Hall edge channels, Phys. Rev. B 88, 085302 (2013).

[75] A. Papoulis, Signal Analysis (Mac-Graww Hill, New York, 1977).

[76] D. W. Bliss and K. W. Forsythe, in Conference record of the Thirty-Seventh Asilomar Conference on Signals, Systems and Computers, November 9-12, Pacific Grove, California (IEEE, Piscataway, N.J., 2003), Vol. 1, p. 54.

[77] G. Raleigh and J. Cioffi, Spatio-temporal coding for wireless communication, IEEE Trans. Commun. 46, 357 (1998).
[78] V. Freulon, A. Marguerite, J. Berroir, B. Plaçais, A. Cavanna, Y. Jin, and G. Fève, Hong-Ou-Mandel experiment for temporal investigation of single-electron fractionalization, Nat. Commun. 6, 6854 (2015).

[79] M. Moskalets, High-temperature fusion of a multielectron Leviton, Phys. Rev. B 97, 155411 (2018).

[80] R. Rodriguez, F. Parmentier, D. Ferraro, P. Roulleau, U. Gensser, A. Cavanna, M. Sassetti, F. Portier, D. Mailly, and P. Roche, Relaxation and revival of quasiparticles injected in an interacting quantum Hall liquid, Nat. Commun. 11, 2426 (2020).

[81] J. Romero, R. Babbush, J. R. McClean, C. Hempel, P. J. Love, and A. Aspuru-Guzik, Strategies for quantum computing molecular energies using the unitary coupled cluster ansatz, Quantum Sci. Technol. 4, 014008 (2018).

[82] D. Dasenbrook and C. Flindt, Dynamical generation and detection of entanglement in neutral Leviton pairs, Phys. Rev. B 92, 161412(R) (2015).

[83] P. Hofer, D. Dasenbrook, and C. Flindt, On-demand entanglement generation using dynamic single electron sources, Phys. Status Solidi (b) 254, 1600582 (2017).

[84] D. Dasenbrook, J. Bowles, J. B. Brask, P. P. Hofer, C. Flindt, and N. Brunner, Single-electron entanglement and nonlocality, New J. Phys. 18, 043036 (2016).

[85] M. Moskalets, Single-particle shot noise at nonzero temperature, Phys. Rev. B 96, 165423 (2017).

[86] M. Moskalets, Single-electron second-order correlation function $G^{(2)}$ at nonzero temperatures, Phys. Rev. B 98, 115421 (2018).

[87] D. Ferraro, C. Wahl, J. Rech, T. Jonckheere, and T. Martin, Electronic Hong-Ou-Mandel interferometry in twodimensional topological insulators, Phys. Rev. B 89, 075407 (2014).

[88] D. Ferraro, T. Jonckheere, J. Rech, and T. Martin, Electronic quantum optics beyond the integer quantum Hall effect, Phys. Status Solidi (b) 254, 1600531 (2017).

[89] F. Ronetti, L. Vannucci, D. Ferraro, T. Jonckheere, J. Rech, T. Martin, and M. Sassetti, Crystallization of Levitons in the fractional quantum Hall regime, Phys. Rev. B 98, 075401 (2018).

[90] G. Gasse, C. Lupien, and B. Reulet, Observation of Squeezing in the Electron Quantum Shot Noise of a Tunnel Junction, Phys. Rev. Lett. 111, 136601 (2013).

[91] J. C. Forgues, C. Lupien, and B. Reulet, Emission of Microwave Photon Pairs by a Tunnel Junction, Phys. Rev. Lett. 113, 043602 (2014).

[92] A. L. Grimsmo, F. Qassemi, B. Reulet, and A. Blais, Quantum Optics Theory of Electronic Noise in Quantum Conductors, Phys. Rev. Lett. 116, 043602 (2016).

[93] S. Virally, J. O. Simoneau, C. Lupien, and B. Reulet, Discrete photon statistics from continuous microwave measurements, Phys. Rev. A 93, 043813 (2016).

[94] D. Ferraro, F. Ronetti, J. Rech, T. Jonckheere, M. Sassetti, and T. Martin, Enhancing photon squeezing one Leviton at a time, Phys. Rev. B 97, 155135 (2018).

[95] R. Fletcher and C. Reeves, Function minimization by conjugate gradients, Comput. J. 7, 149 (1964).

[96] S. E. Nigg and M. Büttiker, Quantum to classical transition of the charge relaxation resistance of a mesoscopic capacitor, Phys. Rev. B 77, 085312 (2008). 